\title{
Highly Efficient Copper-Catalyzed $N$-Arylation of Nitrogen-Containing Heterocycles with Aryl and Heteroaryl
}

\section{Halides}

\author{
Liangbo Zhu, Liang Cheng, Yuxi Zhang, Rugang Xie, and Jingsong You*
}

Key Laboratory of Green Chemistry and Technology of Ministry of Education, College of Chemistry, and State Key Laboratory of Biotherapy, West China Hospital, West China Medical School, Sichuan University, 29 Wangjiang Road, Chengdu 610064, PR China Fax: 86-28-85412203; E-mail: jsyou@scu.edu.cn

\section{Supporting Information}

\section{Table of contents}

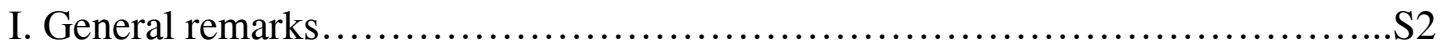

II. General procedure for the catalytic $N$-arylation of nitrogen-containing heterocycles

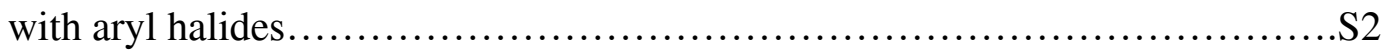

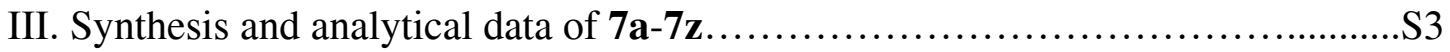

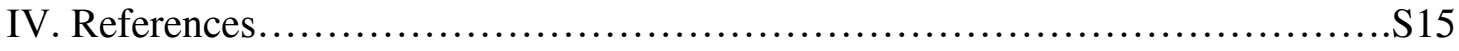

V. Copies of ${ }^{1} \mathrm{H}$ and ${ }^{13} \mathrm{C}$ NMR spectra for compounds $4 \mathbf{a}-\mathbf{4} \mathbf{c}, \mathbf{3 a}-\mathbf{3 b}, \mathbf{2} \mathbf{a}-\mathbf{2} \mathbf{b}$ and

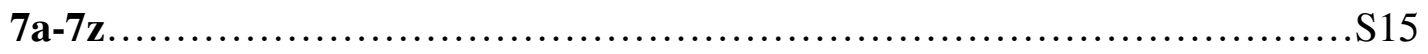




\section{General Remarks}

The ${ }^{1} \mathrm{H}$ NMR (300 MHz, $400 \mathrm{MHz}$ or $600 \mathrm{MHz}$ ) chemical shifts were measured relative to tetramethylsilane, $\mathrm{CDCl}_{3}$ or $\mathrm{CD}_{3} \mathrm{OD}$ as the internal reference. The ${ }^{13} \mathrm{C} \mathrm{NMR}(50 \mathrm{MHz}$, $75 \mathrm{MHz}, 100 \mathrm{MHz}$ or $150 \mathrm{MHz}$ ) chemical shifts are given using $\mathrm{CDCl}_{3}$ or $\mathrm{CD}_{3} \mathrm{OD}$ as the internal standard. Low-resolution mass spectra (MS) were obtained by EI or ESI or GC-MS (EI) and high resolution mass spectra (HRMS) were recorded by FAB with $m$-NBA or EI.

Unless otherwise noted, all reagents were obtained from commercial suppliers and used without further purification. Solvents were dried by refluxing for at least 24 h over $\mathrm{CaH}_{2}$ (chloroform, DMF or DMSO), sodium/benzophenone (THF or toluene), and freshly distilled prior to use. CuI should be washed with THF using a Soxhlet extractor before it is used to ensure satisfactory catalytic activity. Unless otherwise indicated, all syntheses and manipulations were carried out under dry dinitrogen atmosphere.

\section{General procedure for the catalytic $N$-arylation of nitrogen-containing heterocycles with aryl halides}

To a flame-dried Schlenk test tube with a magnetic stirring bar was charged with CuI (9.6 $\mathrm{mg}, 0.05 \mathrm{mmol})$, ligand $(0.1 \mathrm{mmol}), \mathrm{Cs}_{2} \mathrm{CO}_{3}(0.652 \mathrm{~g}, 2.0 \mathrm{mmol})$, nitrogen-containing heterocycle $(1.0 \mathrm{mmol})$, aryl or heteroaryl halide $(1.2 \mathrm{mmol})$ and DMF $(2 \mathrm{~mL})$ under $\mathrm{N}_{2}$. A rubber septum was replaced with a glass stopper, and the system was then evacuated twice and back filled with $\mathrm{N}_{2}$. The reaction mixture was stirred for $30 \mathrm{~min}$ at room temperature, and then heated to $110^{\circ} \mathrm{C}$ for $24 \mathrm{~h}$. The reaction mixture was then cooled to 
ambient temperature, diluted with $2-3 \mathrm{~mL}$ of ethyl acetate, filtered through a plug of silica gel, and washed with $10-20 \mathrm{~mL}$ of ethyl acetate. The filtrate was concentrated and the resulting residue was purified by column chromatography on silica gel to provide the desired product.

\section{Synthesis and analytical data of $7 \mathrm{a}-7 \mathrm{z}$}

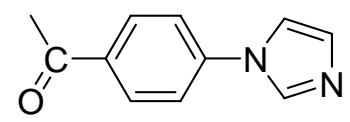

\section{1-(4-Imidazol-1-yl-phenyl)-ethanone (7b) ${ }^{1}$}

Following the general procedure, imidazole $(0.068 \mathrm{~g}, 1.0 \mathrm{mmol})$ was coupled with 4-bromoacetophenone $(0.239 \mathrm{~g}, 1.2 \mathrm{mmol})$ in the presence of ligand 4a. The crude product was purified over a silica gel column using ethyl acetate/petrol ether (1/1) to give a white solid (99\% yield). ${ }^{1} \mathrm{H}$ NMR (600 MHz, $\left.\mathrm{CDCl}_{3}\right): \delta 2.65$ (s, 3H), 7.33 (bs, 1H), $7.45(\mathrm{bs}, 1 \mathrm{H}), 7.51(\mathrm{~d}, J=8.4 \mathrm{~Hz}, 2 \mathrm{H}), 8.05(\mathrm{bs}, 1 \mathrm{H}), 8.09(\mathrm{~d}, J=8.4 \mathrm{~Hz}, 2 \mathrm{H}) \mathrm{ppm} .{ }^{13} \mathrm{C}$ NMR $\left(75 \mathrm{MHz}, \mathrm{CDCl}_{3}\right): \delta 26.6,117.7,120.7,130.3,131.1,135.4,135.7,140.7,196.5$ ppm. GC-MS (EI, $m / z): 186\left[\mathrm{M}^{+}\right]$.

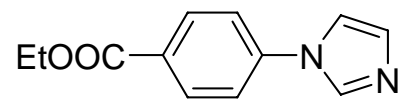

\section{4-Imidazol-1-yl-benzoic acid ethyl ester (7c) ${ }^{1}$}

Following the general procedure, imidazole $(0.068 \mathrm{~g}, 1.0 \mathrm{mmol})$ was coupled with ethyl 4-bromobenzoate $(196 \mu \mathrm{L}, 1.2 \mathrm{mmol})$ in the presence of ligand 4a. The crude product was purified over a silica gel column using ethyl acetate/petrol ether (1/1) to give a white 
solid (61\% yield). ${ }^{1} \mathrm{H}$ NMR (400 MHz, $\left.\mathrm{CDCl}_{3}\right): \delta 1.36(\mathrm{t}, J=7.6 \mathrm{~Hz}, 3 \mathrm{H}), 4.36(\mathrm{~m}, 2 \mathrm{H})$, 7.25 (bs, 1H), 7.36 (bs, 1H), 7.45-7.47 (m, 2H), 7.96 (bs, 1H), 8.11-8.16 (m, 2H) ppm. ${ }^{13} \mathrm{C}$ NMR (75 MHz, $\left.\mathrm{CDCl}_{3}\right): \delta 14.3,61.3,117.9,120.1,120.5,129.4,131.1,131.5,140.6$, 165.5 ppm. GC-MS (EI, $\mathrm{m} / z): 216\left[\mathrm{M}^{+}\right]$

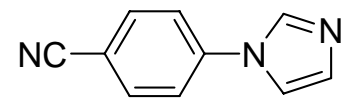

\section{4-Imidazol-1-yl-benzonitrile (7d) ${ }^{2}$}

Following the general procedure, imidazole $(0.068 \mathrm{~g}, 1.0 \mathrm{mmol})$ was coupled with 4-bromobenzonitrile $(0.216 \mathrm{~g}, 1.2 \mathrm{mmol})$ in the presence of ligand $4 \mathbf{a}$. The crude product was purified over a silica gel column using ethyl acetate/petrol ether (1/1) to give a white solid (95\% yield). ${ }^{1} \mathrm{H}$ NMR (600 MHz, $\left.\mathrm{CDCl}_{3}\right): \delta 7.27(\mathrm{~s}, 1 \mathrm{H}), 7.35(\mathrm{~s}, 1 \mathrm{H}), 7.54(\mathrm{~d}, J=$ $8.4 \mathrm{~Hz}, 2 \mathrm{H}), 7.81(\mathrm{~d}, J=8.4 \mathrm{~Hz}, 2 \mathrm{H}), 7.99(\mathrm{~s}, 1 \mathrm{H}) \mathrm{ppm} .{ }^{13} \mathrm{C} \mathrm{NMR}\left(150 \mathrm{MHz}, \mathrm{CDCl}_{3}\right): \delta$ 110.9, 117.5, 117.8, 121.3, 131.4, 134.1, 135.2, 140.4 ppm. GC-MS (EI, $m / z): 169$ [M+'].

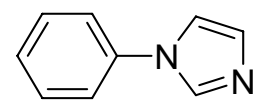

\section{1-Phenyl-1H-imidazole (7a) ${ }^{2}$}

Following the general procedure, imidazole $(0.068 \mathrm{~g}, 1.0 \mathrm{mmol})$ was coupled with bromobenzene ( $126 \mu \mathrm{L}, 1.2 \mathrm{mmol})$ in the presence of ligand $\mathbf{4 a}$. The crude product was purified over a silica gel column using ethyl acetate/petrol ether (1/1) to give pale yellow oil (96\% yield). ${ }^{1} \mathrm{H}$ NMR (600 MHz, $\left.\mathrm{CDCl}_{3}\right): \delta 7.25(\mathrm{~s}, 1 \mathrm{H}), 7.32(\mathrm{~s}, 1 \mathrm{H}), 7.38-7.41(\mathrm{~m}$, 3H), 7.49-7.51 (m, 2H), $7.92(\mathrm{~s}, 1 \mathrm{H}) \mathrm{ppm} .{ }^{13} \mathrm{C} \mathrm{NMR}\left(150 \mathrm{MHz}, \mathrm{CDCl}_{3}\right): \delta 118.3,121.5$, 127.5, 129.9, 130.3, 135.7, 137.3 ppm. GC-MS (EI, $m / z): 144\left[\mathrm{M}^{+}\right]$. 


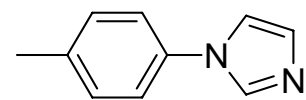

\section{1-(4-Tolyl)-1H-imidazole $(7 e)^{2}$}

Following the general procedure, imidazole $(0.068 \mathrm{~g}, 1.0 \mathrm{mmol})$ was coupled with 4-bromotoluene $(0.205 \mathrm{~g}, 1.2 \mathrm{mmol})$ in the presence of ligand $\mathbf{4 a}$. The crude product was purified over a silica gel column using ethyl acetate/petrol ether (1/1) to give a white solid (89\% yield). ${ }^{1} \mathrm{H}$ NMR (600 MHz, $\left.\mathrm{CDCl}_{3}\right): \delta 2.40$ (s, 3H), $7.19(\mathrm{~s}, 1 \mathrm{H}), 7.25(\mathrm{~s}, 1 \mathrm{H})$, 7.27 (bs, 4H), $7.82(\mathrm{~s}, 1 \mathrm{H}) \mathrm{ppm} .{ }^{13} \mathrm{C} \mathrm{NMR}\left(150 \mathrm{MHz}, \mathrm{CDCl}_{3}\right): \delta$ 20.9, 118.3, 121.3, $130.1,130.3,134.9,135.5,137.4$ ppm. GC-MS (EI, m/z): $158\left[\mathrm{M}^{+}\right]$.

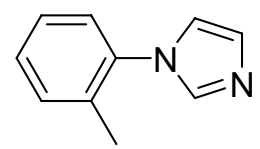

\section{1-(2-Tolyl)-1H-imidazole $\left(7 \mathbf{f}^{1}\right.$}

Following the general procedure, imidazole $(0.068 \mathrm{~g}, 1.0 \mathrm{mmol})$ was coupled with 2-bromotoluene $(0.205 \mathrm{~g}, 1.2 \mathrm{mmol})$ in the presence of ligand $4 \mathbf{a}$. The crude product was purified over a silica gel column using ethyl acetate/petrol ether (1/1) to give pale yellow oil $\left(78 \%\right.$ yield). ${ }^{1} \mathrm{H}$ NMR (400 MHz, $\left.\mathrm{CDCl}_{3}\right): \delta 2.12(\mathrm{~s}, 3 \mathrm{H}), 6.99(\mathrm{t}, J=1.2 \mathrm{~Hz}, 1 \mathrm{H}) 7.13$ $7.31(\mathrm{~m}, 5 \mathrm{H}), 7.51(\mathrm{~s}, 1 \mathrm{H}) \mathrm{ppm} .{ }^{13} \mathrm{C} \mathrm{NMR}\left(100 \mathrm{MHz}, \mathrm{CDCl}_{3}\right): \delta 17.4,120.3,126.3$, 126.6, 128.6, 129.1, 131.1, 133.6, 136.4, 137.3 ppm. GC-MS (EI, m/z): $158\left[\mathrm{M}^{+}\right]$

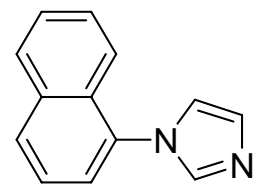




\section{1-Naphthalen-1-yl-1H-imidazole $(7 \mathrm{~g})^{1}$}

Following the general procedure, imidazole $(0.068 \mathrm{~g}, 1.0 \mathrm{mmol})$ was coupled with 1-bromonaphthalene $(167 \mu \mathrm{L}, 1.2 \mathrm{mmol})$ for $24 \mathrm{~h}$ at $110^{\circ} \mathrm{C}$ in the presence of ligand $4 \mathbf{a}$. The crude product was purified over a silica gel column using ethyl acetate/petrol ether (1/1) to give a white solid (66\% yield). ${ }^{1} \mathrm{H}$ NMR $\left(600 \mathrm{MHz}, \mathrm{CDCl}_{3}\right): \delta 7.26$ (bs, $\left.1 \mathrm{H}\right)$, 7.31 (bs, $1 \mathrm{H}), 7.46(\mathrm{~d}, J=7.2 \mathrm{~Hz}, 1 \mathrm{H}), 7.52-7.62(\mathrm{~m}, 4 \mathrm{H}), 7.78(\mathrm{~s}, 1 \mathrm{H}), 7.95-7.97(\mathrm{~m}, 2 \mathrm{H})$ ppm. ${ }^{13} \mathrm{C}$ NMR (150 MHz, $\left.\mathrm{CDCl}_{3}\right): \delta 121.5,122.1,123.5,125.0,126.8,127.4,128.1$, 129.1, 129.3, 129.4, 133.9, 134.0, 138.2 ppm. GC-MS (EI, $m / z): 194\left[\mathrm{M}^{+}\right]$.

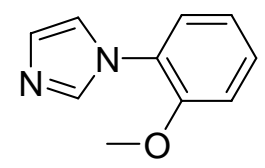

\section{1-(2-Methoxyphenyl)-1H-imidazole (7h) $)^{3}$}

Following the general procedure, imidazole $(0.068 \mathrm{~g}, 1.0 \mathrm{mmol})$ was coupled with 2-bromoanisole ( $149 \mu \mathrm{L}, 1.2 \mathrm{mmol})$ for $48 \mathrm{~h}$ at $110^{\circ} \mathrm{C}$ in the presence of ligand $4 \mathrm{a}$. The crude product was purified over a silica gel column using ethyl acetate/petrol ether (1/1) to give a white solid (64\% yield). ${ }^{1} \mathrm{H}$ NMR (400 MHz, $\mathrm{CDCl}_{3}$ ): $\delta 3.84$ (s, 3H), 6.90 (bs, 1H), 7.02-7.07 (m, 2H), $7.20(\mathrm{bs}, 1 \mathrm{H}), 7.28(\mathrm{~d}, J=7.6 \mathrm{~Hz}, 1 \mathrm{H}), 7.34(\mathrm{t}, J=7.6 \mathrm{~Hz}, 1 \mathrm{H})$, 7.91 (bs, $1 \mathrm{H}$ ) ppm. ${ }^{13} \mathrm{C} \mathrm{NMR}\left(50 \mathrm{MHz}, \mathrm{CDCl}_{3}\right): \delta 55.6,112.2,120.2,120.8,125.3,126.3$, 128.4, 128.8, 137.6, 152.4 ppm. GC-MS (EI, $m / z): 174\left[\mathrm{M}^{+}\right]$.

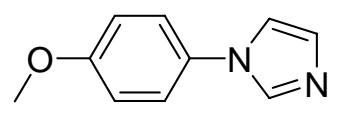

1-(4-Methoxyphenyl)-1H-imidazole $(7 \mathbf{i})^{2}$

Following the general procedure, imidazole $(0.068 \mathrm{~g}, 1.0 \mathrm{mmol})$ was coupled with 
4-bromoanisole $(150 \mu \mathrm{L}, 1.2 \mathrm{mmol})$ in the presence of ligand $4 \mathbf{a}$. The crude product was purified over a silica gel column using ethyl acetate/petrol ether (1/1) to give a white solid (88\% yield). ${ }^{1} \mathrm{H}$ NMR (600 MHz, $\left.\mathrm{CDCl}_{3}\right): \delta 3.86(\mathrm{~s}, 3 \mathrm{H}), 6.99(\mathrm{~d}, J=8.4 \mathrm{~Hz}, 2 \mathrm{H})$, 7.20 (bs, 1H), 7.22 (bs, 1H), 7.30 (d, $J=9.0 \mathrm{~Hz}, 2 \mathrm{H}), 7.79$ (bs, 1H) ppm. ${ }^{13} \mathrm{C}$ NMR (150 $\left.\mathrm{MHz}, \mathrm{CDCl}_{3}\right): \delta 55.6,114.8,118.7,123.18,130.0,130.7,135.8,158.9$ ppm. GC-MS (EI, $m / z): 174\left[\mathrm{M}^{+}\right]$.

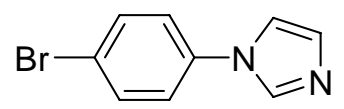

\section{1-(4-Bromo-phenyl)-1H-imidazole $(7 \mathbf{j})^{1}$}

Following the general procedure, imidazole $(0.068 \mathrm{~g}, 1.0 \mathrm{mmol})$ was coupled with 1,4-dibromobenzene $(0.283 \mathrm{~g}, 1.2 \mathrm{mmol})$ in the presence of ligand $4 \mathbf{a}$. The crude product was purified over a silica gel column using ethyl acetate/petrol ether (1/1) to give a white solid (87\% yield). ${ }^{1} \mathrm{H}$ NMR (600 MHz, $\left.\mathrm{CDCl}_{3}\right): \delta$ 7.23-7.30 (m, 4H), $7.61(\mathrm{~m}, 2 \mathrm{H}), 7.85$ (bs, $1 \mathrm{H})$ ppm. ${ }^{13} \mathrm{C}$ NMR $\left(150 \mathrm{MHz}, \mathrm{CDCl}_{3}\right): \delta 118.0,120.8,122.9,130.7,132.9,135.4$, 136.3 ppm. GC-MS (EI, $m / z): 222\left[\mathrm{M}^{+}\right]$.

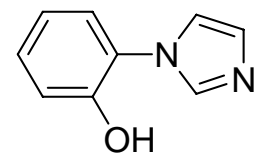

\section{2-Imidazol-1-yl-phenol (7k) ${ }^{3}$}

Following the general procedure, imidazole $(0.068 \mathrm{~g}, 1.0 \mathrm{mmol})$ was coupled with 2-bromophenol $(127 \mu \mathrm{L}, 1.2 \mathrm{mmol})$ in the presence of ligand $4 \mathbf{a}$ for $48 \mathrm{~h}$. The crude reaction mixture was dissolved in $20 \mathrm{~mL}$ of $2 \mathrm{M} \mathrm{HCl}$, and washed once with diethyl ether. The aqueous layer was brought to $\mathrm{pH}=7.5$ with $\mathrm{NaHCO}_{3}$, and extracted repeatedly with 
$\mathrm{CH}_{2} \mathrm{Cl}_{2}$. The combined organic layer was dried with anhydrous $\mathrm{MgSO}_{4}$, and concentrated under reduced pressure. The crude product was purified over a silica gel column using ethanol/ethyl acetate (1/100) to give a white solid (61\% yield). ${ }^{1} \mathrm{H}$ NMR (400 MHz, $\left.\mathrm{CD}_{3} \mathrm{OD}\right): \delta 6.92(\mathrm{t}, J=7.6 \mathrm{~Hz}, 1 \mathrm{H}), 7.11(\mathrm{~d}, J=8.4 \mathrm{~Hz}, 1 \mathrm{H}), 7.21(\mathrm{t}, J=8.0 \mathrm{~Hz}, 1 \mathrm{H})$, $7.30(\mathrm{~d}, J=7.6 \mathrm{~Hz}, 1 \mathrm{H}), 7.52(\mathrm{~s}, 1 \mathrm{H}), 8.34$ (bs, $1 \mathrm{H}) \mathrm{ppm} .{ }^{13} \mathrm{C} \mathrm{NMR}\left(150 \mathrm{MHz}, \mathrm{CD}_{3} \mathrm{OD}\right.$ and $\left.\mathrm{CDCl}_{3}\right): \delta 116.7,119.3,124.7,128.1,149.9 \mathrm{ppm} . \mathrm{MS}\left(\mathrm{ESI}^{+}\right) \mathrm{m} / z .161[\mathrm{M}+1]^{+}$.

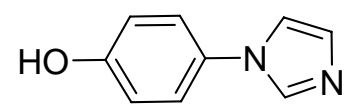

\section{4-Imidazol-1-ylphenol (7l) ${ }^{1}$}

Following the general procedure, imidazole $(0.068 \mathrm{~g}, 1.0 \mathrm{mmol})$ was coupled with 4-bromophenol ( $0.207 \mathrm{~g}, 1.2 \mathrm{mmol})$ in the presence of ligand 4a. The crude reaction mixture was dissolved in $20 \mathrm{~mL}$ of $2 \mathrm{M} \mathrm{HCl}$, and washed once with diethyl ether. The aqueous layer was brought to $\mathrm{pH}=8$ with $\mathrm{Na}_{2} \mathrm{CO}_{3}$, and extracted repeatedly with $\mathrm{CH}_{2} \mathrm{Cl}_{2}$. The combined organic layer was dried with anhydrous $\mathrm{MgSO}_{4}$, and concentrated under reduced pressure. The crude product was purified over a silica gel column using ethanol/ ethyl acetate (1/100) to give a white solid (70\% yield). ${ }^{1} \mathrm{H}$ NMR (600 MHz, $\left.\mathrm{CD}_{3} \mathrm{OD}\right): \delta$ $6.89(\mathrm{~d}, J=8.4 \mathrm{~Hz}, 2 \mathrm{H}), 7.11$ (bs, 1H), 7.33 (d, $J=9.0 \mathrm{~Hz}, 2 \mathrm{H}), 7.44$ (bs, 1H), 7.97 (bs, 1H) ppm. ${ }^{13} \mathrm{C}$ NMR $\left(150 \mathrm{MHz}, \mathrm{CD}_{3} \mathrm{OD}\right): \delta 117.3,120.3,124.2,129.7,130.7,137.1$, $158.5 \mathrm{ppm}$. GC-MS (EI, $\mathrm{m} / z): 160\left[\mathrm{M}^{+}\right]$.

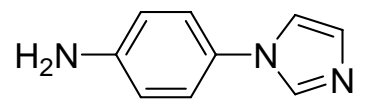

\section{4-Imidazol-1-yl-phenylamine (7m)}


Following the general procedure, imidazole $(0.068 \mathrm{~g}, 1.0 \mathrm{mmol})$ was coupled with 4-bromoaniline ( $0.206 \mathrm{~g}, 1.2 \mathrm{mmol})$ in the presence of ligand 4a. The crude product was purified over a silica gel column using ethyl acetate/petrol ether (1/1) to give a white solid (82\% yield). ${ }^{1} \mathrm{H}$ NMR (600 MHz, $\left.\mathrm{CDCl}_{3}\right): \delta 3.58$ (bs, 2H), $6.74(\mathrm{~s}, 1 \mathrm{H}), 6.75(\mathrm{~s}, 1 \mathrm{H})$, 7.15-7.27 (m, 4H), $7.74(\mathrm{~s}, 1 \mathrm{H}) \mathrm{ppm} .{ }^{13} \mathrm{C} \mathrm{NMR}\left(150 \mathrm{MHz}, \mathrm{CDCl}_{3}\right): \delta 115.5,118.8,123.3$, 128.7, 129.7, 135.8, $146.1 \mathrm{ppm}$. GC-MS (EI, $m / z): 159\left[\mathrm{M}^{+}\right]$.

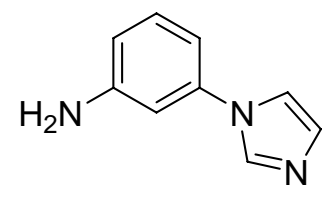

\section{3-Imidazol-1-yl-phenylamine $(\mathbf{7 n})^{1}$}

Following the general procedure, imidazole $(0.068 \mathrm{~g}, 1.0 \mathrm{mmol})$ was coupled with 3-bromoaniline $(131 \mu \mathrm{L}, 1.2 \mathrm{mmol})$ in the presence of ligand 4a. The crude product was purified over a silica gel column using ethyl acetate/petrol ether (1/1) to give a light yellow solid (85\% yield). ${ }^{1} \mathrm{H}$ NMR (400 MHz, $\left.\mathrm{CDCl}_{3}\right): \delta 4.00(\mathrm{bs}, 2 \mathrm{H}), 6.63-6.65(\mathrm{~m}$, $2 \mathrm{H})$, 6.71-6.73 (m, 1H), 7.17-7.23 (m, 3H), $7.81(\mathrm{~s}, 1 \mathrm{H}) \mathrm{ppm} .{ }^{13} \mathrm{C}$ NMR $(75 \mathrm{MHz}$, $\left.\mathrm{CDCl}_{3}\right): \delta 107.7,111.1,113.9,118.3,130.0,130.6,135.6,138.3,148.1 \mathrm{ppm} . \mathrm{GC}-\mathrm{MS}$ (EI, $m / z): 159\left[\mathrm{M}^{+}\right]$.

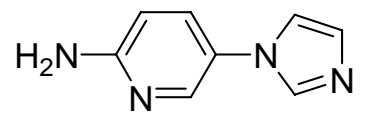

\section{5-(1H-Imidazol-1-yl)pyridin-2-amine (7o)}

Following the general procedure, imidazole $(0.068 \mathrm{~g}, 1.0 \mathrm{mmol})$ was coupled with 
2-amino-5-bromopyridine $(0.208 \mathrm{~g}, 1.2 \mathrm{mmol})$ in the presence of ligand $4 \mathrm{a}$. The crude product was purified over a silica gel column using ethyl acetate to give a light yellow solid (83\% yield). ${ }^{1} \mathrm{H}$ NMR (400 MHz, $\left.\mathrm{CDCl}_{3}\right): \delta 4.74(\mathrm{bs}, 2 \mathrm{H}), 6.57(\mathrm{~d}, J=8.8 \mathrm{~Hz}, 1 \mathrm{H})$, 7.14 (s, 1H), $7.19(\mathrm{~s}, 1 \mathrm{H}), 7.42(2 \mathrm{~d}, J=2.8,2.4 \mathrm{~Hz}, 1 \mathrm{H}), 7.69(\mathrm{~s}, 1 \mathrm{H}), 8.12(\mathrm{~d}, J=2.4 \mathrm{~Hz}$, 1H) ppm. ${ }^{13} \mathrm{C}$ NMR (75 MHz, $\left.\mathrm{CDCl}_{3}\right): \delta 108.7,119.5,125.7,130.3,132.5,136.2,142.1$, $158.2 \mathrm{ppm}$. GC-MS (EI, $\mathrm{m} / z): 160\left[\mathrm{M}^{+}\right]$.

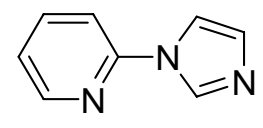

\section{2-(1H-imidazolyl)pyridine $(7 p)^{2}$}

Following the general procedure, imidazole $(0.068 \mathrm{~g}, 1.0 \mathrm{mmol})$ was coupled with 2-bromopyridine ( $117 \mu \mathrm{L}, 1.2 \mathrm{mmol}$ ) in the presence of ligand $4 \mathbf{a}$. The crude product was purified over a silica gel column using ethyl acetate/petrol ether (1/1) to give pale yellow oil $\left(98 \%\right.$ yield) . ${ }^{1} \mathrm{H}$ NMR $\left(300 \mathrm{MHz}, \mathrm{CDCl}_{3}\right): \delta 7.11$ (bs, $\left.1 \mathrm{H}\right), 7.14-7.17(\mathrm{~m}, 1 \mathrm{H}), 7.26$ (d, $J=8.2 \mathrm{~Hz}, 1 \mathrm{H}), 7.53(\mathrm{~s}, 1 \mathrm{H}), 7.71-7.77(\mathrm{~m}, 1 \mathrm{H}), 8.28(\mathrm{bs}, 1 \mathrm{H}), 8.39(2 \mathrm{~d}, J=1.4,1.5$ $\mathrm{Hz}, 1 \mathrm{H}) \mathrm{ppm} .{ }^{13} \mathrm{C} \mathrm{NMR}\left(75 \mathrm{MHz}, \mathrm{CDCl}_{3}\right): \delta 112.3,116.1,121.5,122.0,130.6,135.0$, 139.0, 149.1 ppm. GC-MS (EI, $m / z): 145\left[\mathrm{M}^{+}\right]$.

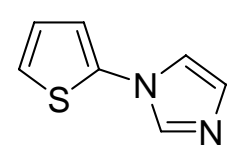

\section{1-(thiophen-2-yl)-1H-imidazole (7q) ${ }^{4}$}

Following the general procedure, imidazole $(0.068 \mathrm{~g}, 1.0 \mathrm{mmol})$ was coupled with 2-bromothiophene (115 $\mu \mathrm{L}, 1.2 \mathrm{mmol})$ in the presence of ligand $\mathbf{4 a}$. The crude product was 
purified over a silica gel column using ethyl acetate/petrol ether (1/1) to give pale yellow oil (75\% yield) . ${ }^{1} \mathrm{H}$ NMR (400 MHz, $\left.\mathrm{CDCl}_{3}\right): \delta 6.94-6.97(\mathrm{~m}, 2 \mathrm{H}), 7.10(2 \mathrm{~d}, J=1.6,2.0$ $\mathrm{Hz}, 1 \mathrm{H}), 7.13(\mathrm{~s}, 1 \mathrm{H}), 7.16(\mathrm{t}, J=1.2 \mathrm{~Hz}, 1 \mathrm{H}), 7.72(\mathrm{~s}, 1 \mathrm{H}) \mathrm{ppm} .{ }^{13} \mathrm{C}$ NMR $(100 \mathrm{MHz}$, $\left.\mathrm{CDCl}_{3}\right): \delta 118.9,120.2,121.7,126.3,130.2,137.0,139.1 \mathrm{ppm} . \mathrm{GC}-\mathrm{MS}(\mathrm{EI}, \mathrm{m} / z): 150$ $\left[\mathrm{M}^{+}\right]$

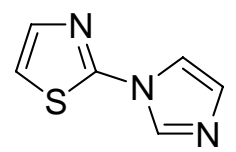

\section{1-(thiazol-2-yl)-1H-imidazole (7r)}

Following the general procedure, imidazole $(0.068 \mathrm{~g}, 1.0 \mathrm{mmol})$ was coupled with 2-bromothiazole (106 $\mu \mathrm{L}, 1.2 \mathrm{mmol})$ in the presence of ligand $4 \mathbf{a}$. The crude product was purified over a silica gel column using ethyl acetate/petrol ether (1/1) to give pale yellow oil (85\% yield) . ${ }^{1} \mathrm{H}$ NMR (400 MHz, $\left.\mathrm{CDCl}_{3}\right): \delta 7.11-7.14(\mathrm{~m}, 2 \mathrm{H}), 7.47(\mathrm{t}, J=1.2 \mathrm{~Hz}$, $1 \mathrm{H}), 7.53(\mathrm{~d}, J=3.6 \mathrm{~Hz}, 1 \mathrm{H}), 8.15(\mathrm{~s}, 1 \mathrm{H}) \mathrm{ppm} .{ }^{13} \mathrm{C} \mathrm{NMR}\left(150 \mathrm{MHz}, \mathrm{CDCl}_{3}\right): \delta 115.5$, 117.6, 130.8, 135.4, 140.5, 157.4 ppm. GC-MS (EI, m/z): $151\left[\mathrm{M}^{+}\right]$.<smiles>c1cnc(-n2ccnc2)nc1</smiles>

\section{2-(1H-imidazol-1-yl)pyrimidine $(7 \mathrm{~s})^{5}$}

Following the general procedure, imidazole $(0.068 \mathrm{~g}, 1.0 \mathrm{mmol})$ was coupled with 2-chloropyrimidine (137mg, $1.2 \mathrm{mmol}$ ) in the presence of ligand $4 \mathbf{a}$. The crude product was purified over a silica gel column using ethyl acetate/petrol ether (1/1) to give white solid (97\% yield). ${ }^{1} \mathrm{H}$ NMR (400 MHz, $\left.\mathrm{CDCl}_{3}\right): \delta 7.10(\mathrm{~s}, 1 \mathrm{H}), 7.14(\mathrm{t}, J=4.8 \mathrm{~Hz}, 1 \mathrm{H}), 7.82(\mathrm{~s}$, $1 \mathrm{H}), 8.55(\mathrm{~s}, 1 \mathrm{H}), 8.61(\mathrm{~d}, J=5.2 \mathrm{~Hz}, 2 \mathrm{H}) \mathrm{ppm} .{ }^{13} \mathrm{C} \mathrm{NMR}\left(100 \mathrm{MHz}, \mathrm{CDCl}_{3}\right): \delta 116.4$, 
118.7, 130.5, 136.0, 158.6 ppm. GC-MS (EI, $m / z): 146\left[\mathrm{M}^{+}\right]$.

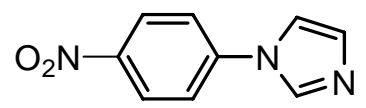

\section{1-(4-Nitrophenyl)-1H-imidazole $(7 \mathrm{t})^{2}$}

Following the general procedure, imidazole $(0.068 \mathrm{~g}, 1.0 \mathrm{mmol})$ was coupled with 1-chloro-4-nitrobenzene $(0.189 \mathrm{~g}, 1.2 \mathrm{mmol})$ in the presence of ligand 4a. The crude product was purified over a silica gel column using ethyl acetate/petrol ether (1/1) to give a pale yellow solid (98\% yield). ${ }^{1} \mathrm{H}$ NMR $\left(600 \mathrm{MHz}, \mathrm{CDCl}_{3}\right): \delta 7.30(\mathrm{~s}, 1 \mathrm{H}), 7.39(\mathrm{~s}, 1 \mathrm{H})$, $7.59(\mathrm{~d}, J=9.0 \mathrm{~Hz}, 2 \mathrm{H}), 8.04(\mathrm{~s}, 1 \mathrm{H}), 8.39(\mathrm{~d}, J=9.0 \mathrm{~Hz}, 2 \mathrm{H}) \mathrm{ppm} .{ }^{13} \mathrm{C}$ NMR $(150 \mathrm{MHz}$, $\left.\mathrm{CDCl}_{3}\right): \delta 117.6,121.0,125.7,131.7,135.4,141.9,146.2$ ppm. GC-MS (EI, m/z): 189 $\left[\mathrm{M}^{+}\right]$

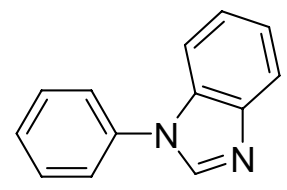

\section{1-Phenyl-1H-benzimidazole $(7 \mathbf{u})^{6}$}

Following the general procedure, benzimidazole $(0.118 \mathrm{~g}, 1.0 \mathrm{mmol})$ was coupled with bromobenzene $(126 \mu \mathrm{L}, 1.2 \mathrm{mmol})$ in the presence of ligand $4 \mathbf{a}$. The crude product was purified over a silica gel column using ethyl acetate/petrol ether (1/1) to give a white solid (75\% yield). ${ }^{1} \mathrm{H}$ NMR (600 $\left.\mathrm{MHz}, \mathrm{CDCl}_{3}\right): \delta 7.33-7.37(\mathrm{~m}, 2 \mathrm{H}), 7.47(\mathrm{t}, J=7.2 \mathrm{~Hz}$, 1H), 7.52-7.60 (m, 5H), 7.89-7.90 (m, 1H), $8.15(\mathrm{~s}, 1 \mathrm{H})$ ppm. ${ }^{13} \mathrm{C}$ NMR $(150 \mathrm{MHz}$, $\left.\mathrm{CDCl}_{3}\right): \delta 110.4,120.5,122.8,123.6,124.0,128.0,130.0,133.6,136.3,142.2,143.9$ ppm. GC-MS (EI, $m / z): 194\left[\mathrm{M}^{+}\right]$. 


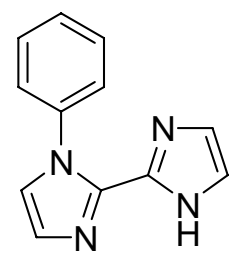

\section{2-(1H-Imidazol-2-yl)-1-phenyl-1H-imidazole (7v)}

Following the general procedure, 2-(1H-imidazol-2-yl)-1H-imidazole $(0.134 \mathrm{~g}, 1.0 \mathrm{mmol})$ was coupled with bromobenzene $(126 \mu \mathrm{L}, 1.2 \mathrm{mmol})$ in the presence of ligand $4 \mathbf{a}$. The crude product was purified over a silica gel column using ethyl acetate/petrol ether (1/1) to give a pale yellow solid (62\% yield). ${ }^{1} \mathrm{H} \mathrm{NMR}\left(600 \mathrm{MHz}, \mathrm{CDCl}_{3}\right): \delta 7.03(\mathrm{~s}, 2 \mathrm{H}), 7.13$ $(\mathrm{s}, 1 \mathrm{H}), 7.21(\mathrm{~s}, 1 \mathrm{H}), 7.44-7.46(\mathrm{~m}, 2 \mathrm{H}), 7.48-7.50(\mathrm{~m}, 3 \mathrm{H}) \mathrm{ppm} .{ }^{13} \mathrm{C}$ NMR $(150 \mathrm{MHz}$, $\left.\mathrm{CDCl}_{3}\right): \delta 123.4,126.3,127.7,128.6,128.9,138.1,138.1,139.2$ ppm. GC-MS (EI, $\left.m / z\right):$ $209[\mathrm{M}-1]^{+}$.

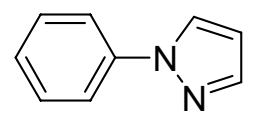

\section{1-Phenyl-1H-pyrazole $(7 \mathbf{w})^{7}$}

Following the general procedure, $1 \mathrm{H}-$ pyrazole $(0.068 \mathrm{~g}, 1.0 \mathrm{mmol})$ was coupled with bromobenzene $(126 \mu \mathrm{L}, 1.2 \mathrm{mmol})$ in the presence of ligand $4 \mathbf{a}$. The crude product was purified over a silica gel column using ethyl acetate/petrol ether (1/8) to give pale yellow oil (87\% yield). ${ }^{1} \mathrm{H} \mathrm{NMR}\left(400 \mathrm{MHz}, \mathrm{CDCl}_{3}\right): \delta 6.43(\mathrm{t}, J=2.4 \mathrm{~Hz}, 1 \mathrm{H}), 7.24-7.28(\mathrm{~m}$, 1H), 7.39-7.45 (m, 2H), 7.68-7.74 (m, 3H), $7.88(\mathrm{~d}, J=2.4 \mathrm{~Hz}, 1 \mathrm{H}) \mathrm{ppm} ;{ }^{13} \mathrm{C}$ NMR $(75$ $\left.\mathrm{MHz}, \mathrm{CDCl}_{3}\right): \delta 107.6,119.2,126.4,126.8,129.4,140.2,141.1 \mathrm{ppm}$. GC-MS (EI, m/z): $144\left[\mathrm{M}^{+}\right]$. 


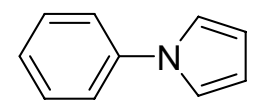

\section{1-Phenyl-1H-pyrrole $(7 x)^{8}$}

Following the general procedure, 1H-pyrrole $(69 \mu \mathrm{L}, 1.0 \mathrm{mmol})$ was coupled with bromobenzene $(126 \mu \mathrm{L}, 1.2 \mathrm{mmol})$ in the presence of ligand $4 \mathbf{a}$. The crude product was purified over a silica gel column using ethyl acetate/petrol ether (1/10) to give a white solid (72\% yield). ${ }^{1} \mathrm{H}$ NMR (400 MHz, $\left.\mathrm{CDCl}_{3}\right): \delta 6.43(\mathrm{t}, J=2.0 \mathrm{~Hz} 2 \mathrm{H}), 7.17(\mathrm{t}, J=2.0$ $\mathrm{Hz} 2 \mathrm{H}), 7.26-7.33(\mathrm{~m}, 1 \mathrm{H}), 7.45-7.51(\mathrm{~m}, 4 \mathrm{H}) \mathrm{ppm} .{ }^{13} \mathrm{C} \mathrm{NMR}\left(75 \mathrm{MHz}, \mathrm{CDCl}_{3}\right): \delta 110.5$, 119.4, 120.6, 125.7, 129.6, 140.9 ppm. GC-MS (EI, $m / z): 143\left[\mathrm{M}^{+}\right]$.<smiles>c1ccc(-n2cncn2)cc1</smiles>

\section{1-Phenyl-1H-1,2,4-triazole $(7 y)^{8}$}

Following the general procedure, 1,2,4-triazole $(0.069 \mathrm{~g}, 1.0 \mathrm{mmol})$ was coupled with iodobenzene $(134 \mu \mathrm{L}, 1.2 \mathrm{mmol})$ at $90{ }^{\circ} \mathrm{C}$ in the presence of ligand $4 \mathbf{a}$. The crude product was purified over a silica gel column using ethyl acetate/petrol ether (1/2) to give a light yellow solid (96\% yield). ${ }^{1} \mathrm{H}$ NMR $\left(300 \mathrm{MHz}, \mathrm{CDCl}_{3}\right): \delta 7.27(\mathrm{t}, J=7.0 \mathrm{~Hz}, 1 \mathrm{H}), 7.38$ (t, $J=8.0 \mathrm{~Hz}, 2 \mathrm{H}), 7.57(\mathrm{~d}, J=8.3 \mathrm{~Hz}, 2 \mathrm{H}), 8.01(\mathrm{bs}, 1 \mathrm{H}), 8.48(\mathrm{bs}, 1 \mathrm{H}) \mathrm{ppm} .{ }^{13} \mathrm{C} \mathrm{NMR}(75$ $\left.\mathrm{MHz}_{\mathrm{CDCl}}\right): \delta 119.6,128.2,129.7,137.0,140.9,152.6$ ppm. GC-MS (EI, m/z): 145 $\left[\mathrm{M}^{+}\right]$

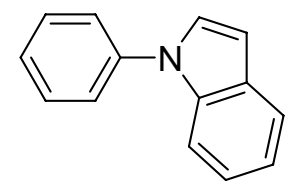

\section{1-Phenyl-1H-indole $(7 z)^{8}$}


Following the general procedure, $1 \mathrm{H}$-indole $(0.117 \mathrm{~g}, 1.0 \mathrm{mmol})$ was coupled with iodobenzene $(134 \mu \mathrm{L}, 1.2 \mathrm{mmol})$ in the presence of ligand $\mathbf{4 a}$. The crude product was purified over a silica gel column using ethyl acetate/petrol ether (1/50) to give colorless oil (98\% yield). ${ }^{1} \mathrm{H}$ NMR (300 MHz, $\left.\mathrm{CDCl}_{3}\right): \delta 6.56(\mathrm{~d}, J=3.2 \mathrm{~Hz}, 1 \mathrm{H}), 7.03-7.13(\mathrm{~m}$, 2H), 7.18-7.25 (m, 2H), 7.33-7.37 (m, 4H), 7.41-7.60 (m, 2H) ppm. ${ }^{13} \mathrm{C}$ NMR (75 MHz, $\left.\mathrm{CDCl}_{3}\right): \delta 103.7,110.6,120.5,121.3,122.5,124.5,126.5,128.0,129.5,130.1,136.0$, 140.0 ppm. GC-MS (EI, m/z): $193\left[\mathrm{M}^{+}\right]$.

\section{References}

(1) Altman, R. A.; Buchwald, S. L. Org. Lett., 2006, 8, 2779-2782.

(2) Choudary, B. M.; Sridhar, C.; Kantam, M. L.; Venkanna, G. T.; Sreedhar, B. J. Am. Chem. Soc. 2005, 127, 9948-9949.

(3) Collman, J. P.; Wang, Z.; Zhong, M.; Zeng, L. J. Chem. Soc., Perkin Trans. 1, 2000, 1217-1221.

(4) Lv, X.; Wang, Z.; Bao, W. Tetrahedron 2006, 62, 4756-4761.

(5) Xie, Y.; Pi, S; Wang, J; Yin, D.; Li, J. J. Org. Chem. 2006, 71, 8324-8327.

(6) Nishiura, K.; Urawa, Y.; Sodab, S. Adv. Synth. Catal. 2004, 346, 1679-1684.

(7) Cristau, H. J.; Cellier, P. P.; Spindler, J. F.; Taillefer, M. Eur. J. Org. Chem. 2004, 695-709.

(8) Cristau, H. J.; Cellier, P. P.; Spindler, J. F.; Taillefer, M. Chem.-Eur. J. 2004, 10, 5607-5622.

\section{Copies of ${ }^{1} \mathrm{H}$ and ${ }^{13} \mathrm{C}$ NMR spectra for compounds $4 \mathrm{a}-4 \mathrm{c}, 3 \mathrm{a}-3 \mathrm{~b}, 2 \mathrm{a}-2 \mathrm{~b}$ and $7 \mathrm{a}-\mathrm{z}$}




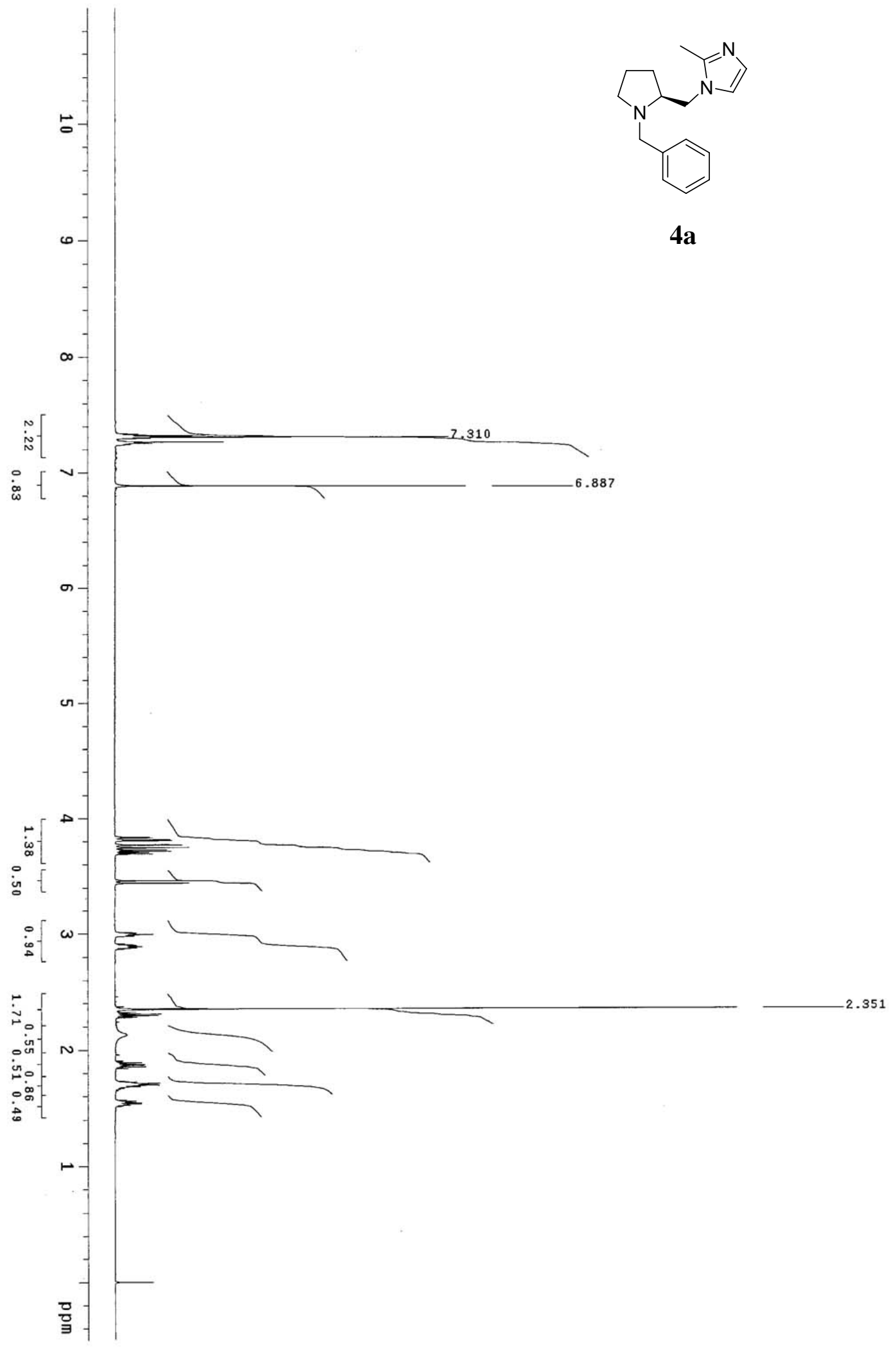




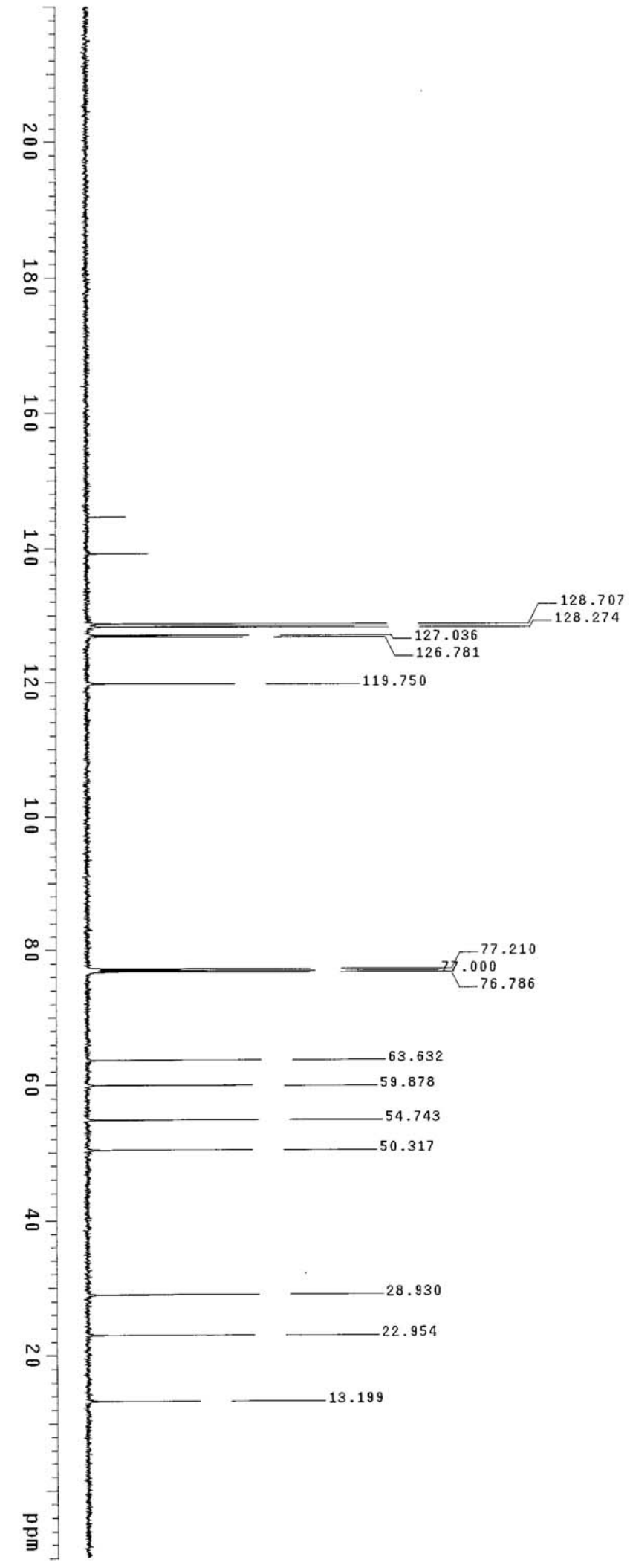

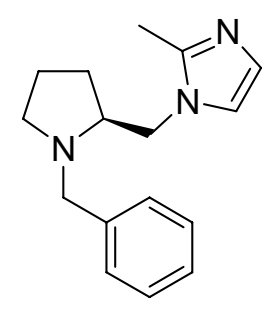

$4 a$ 


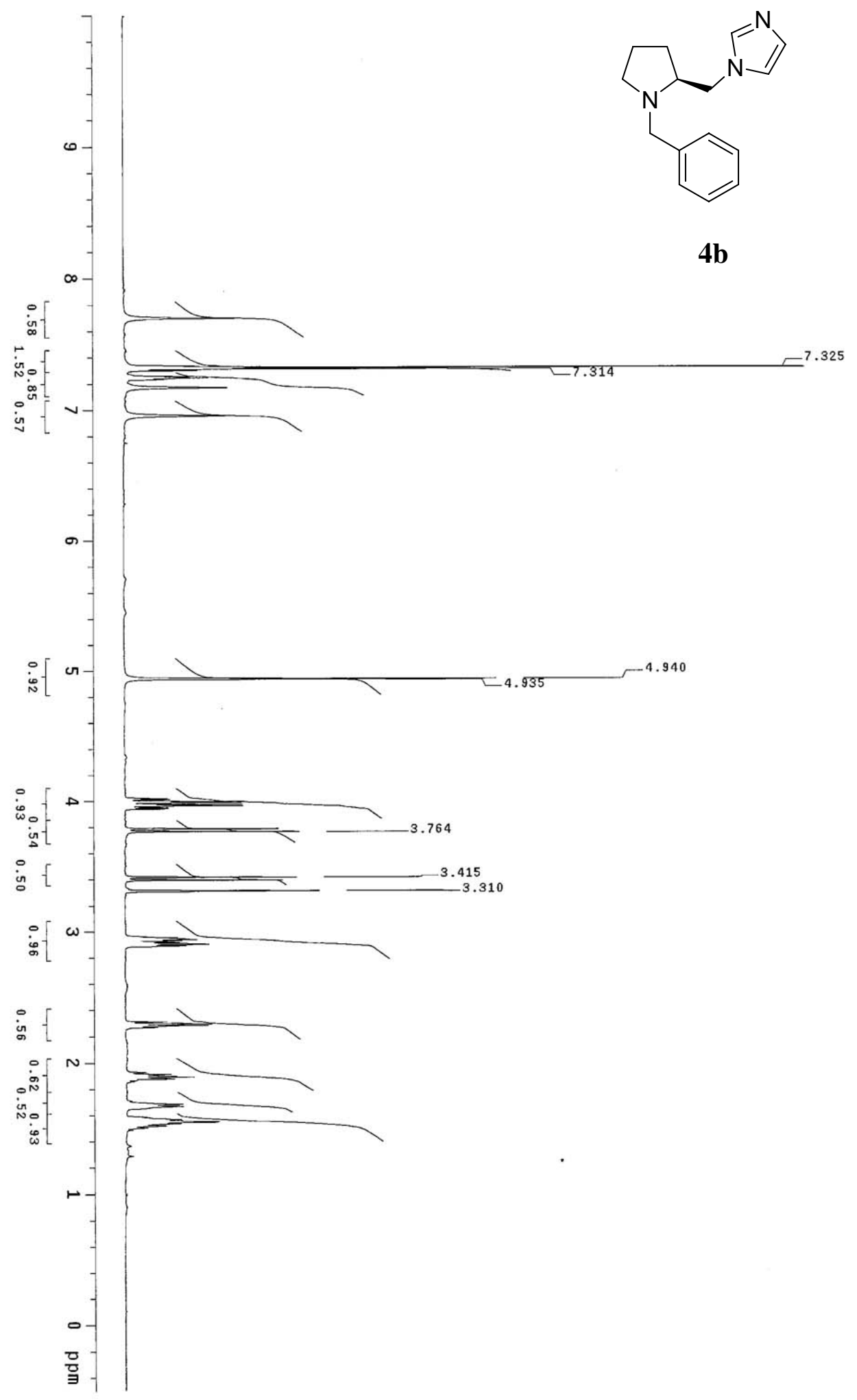




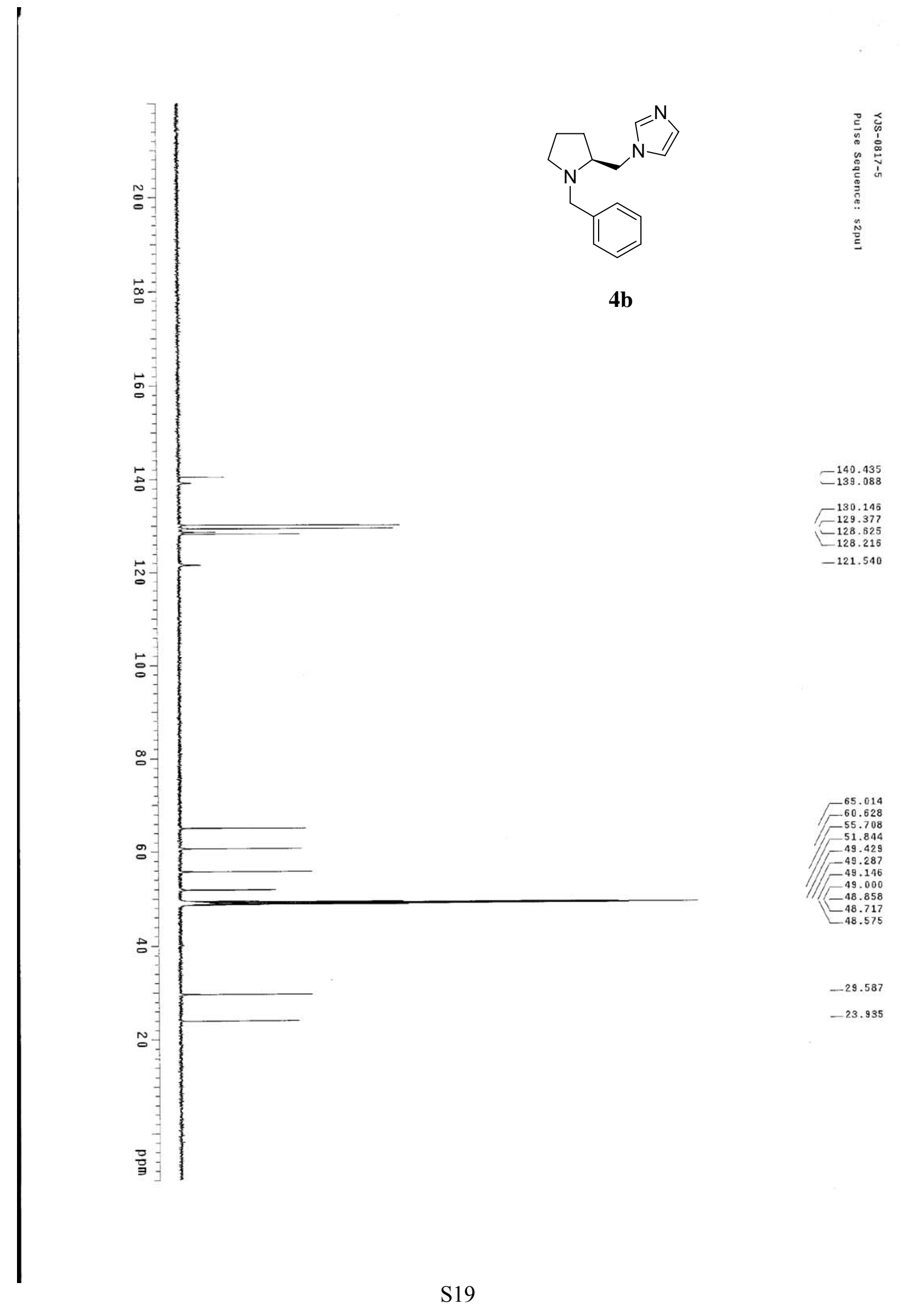




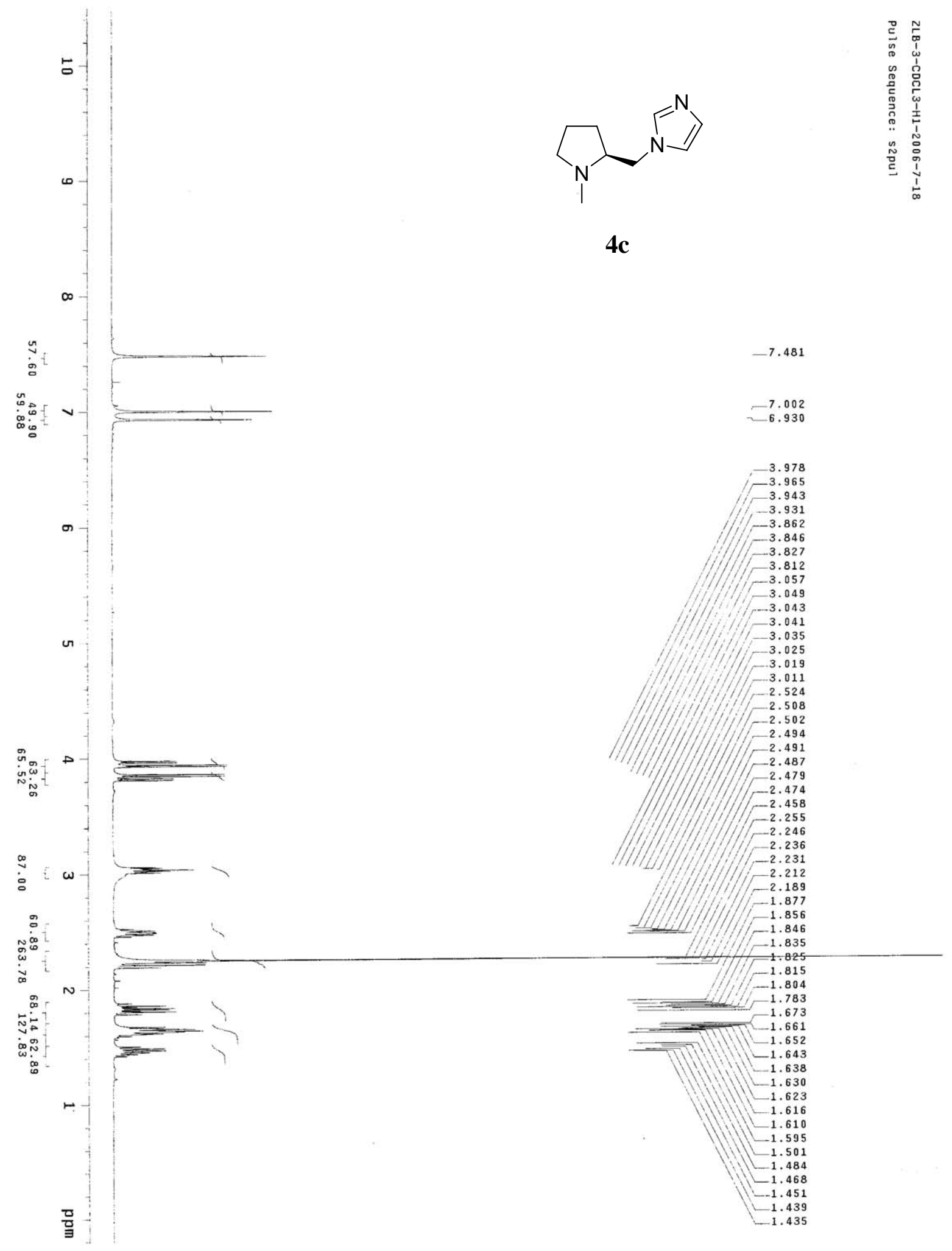




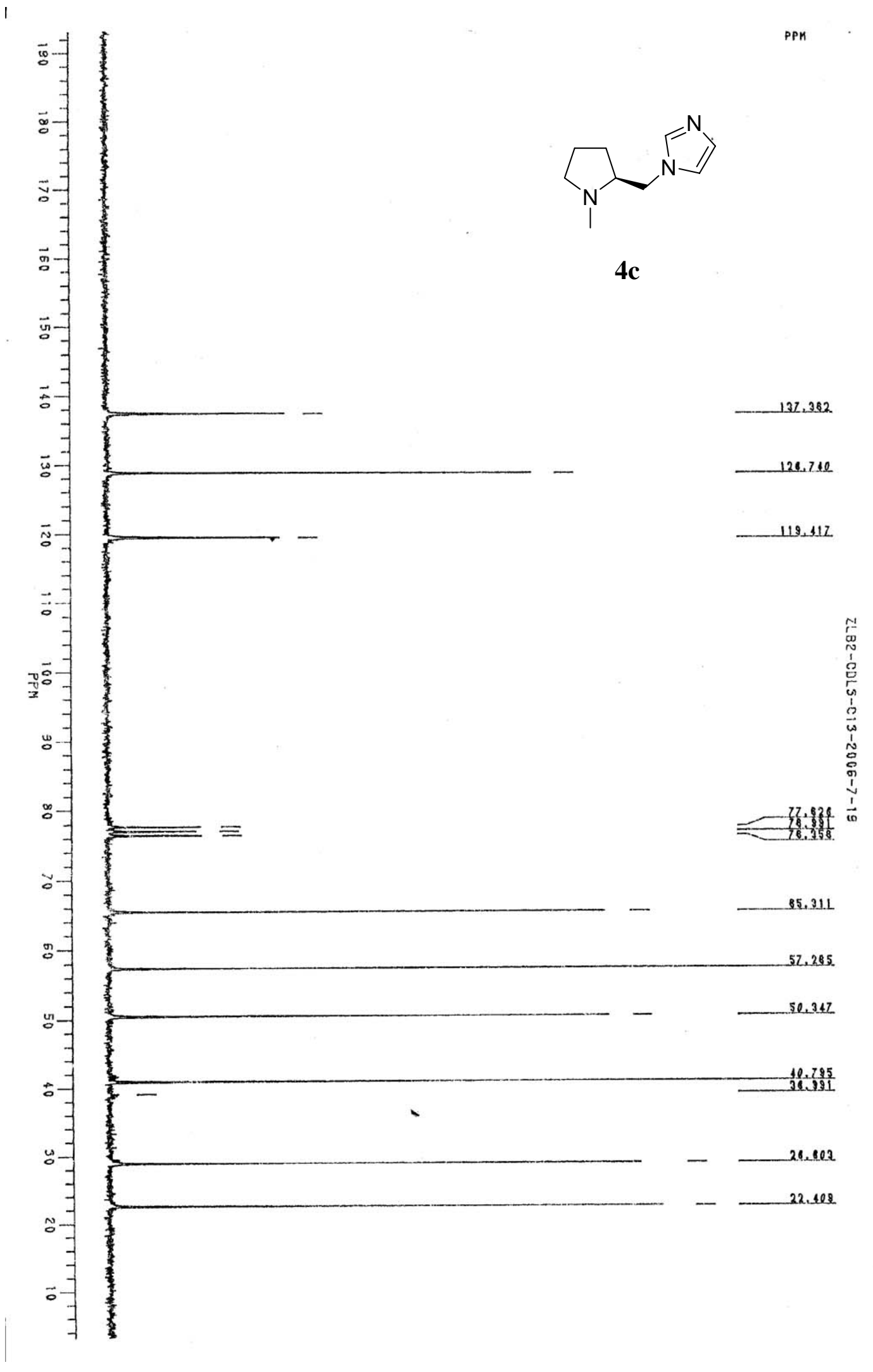




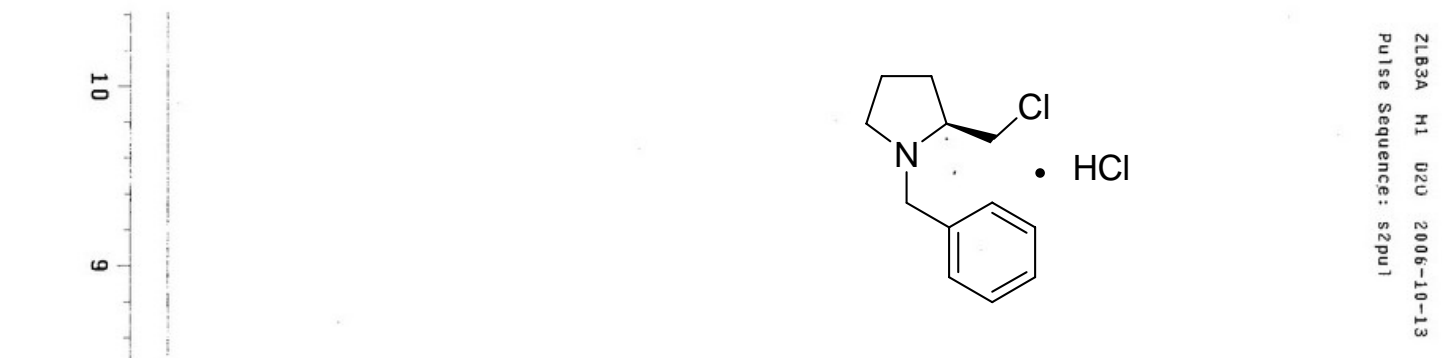

3a

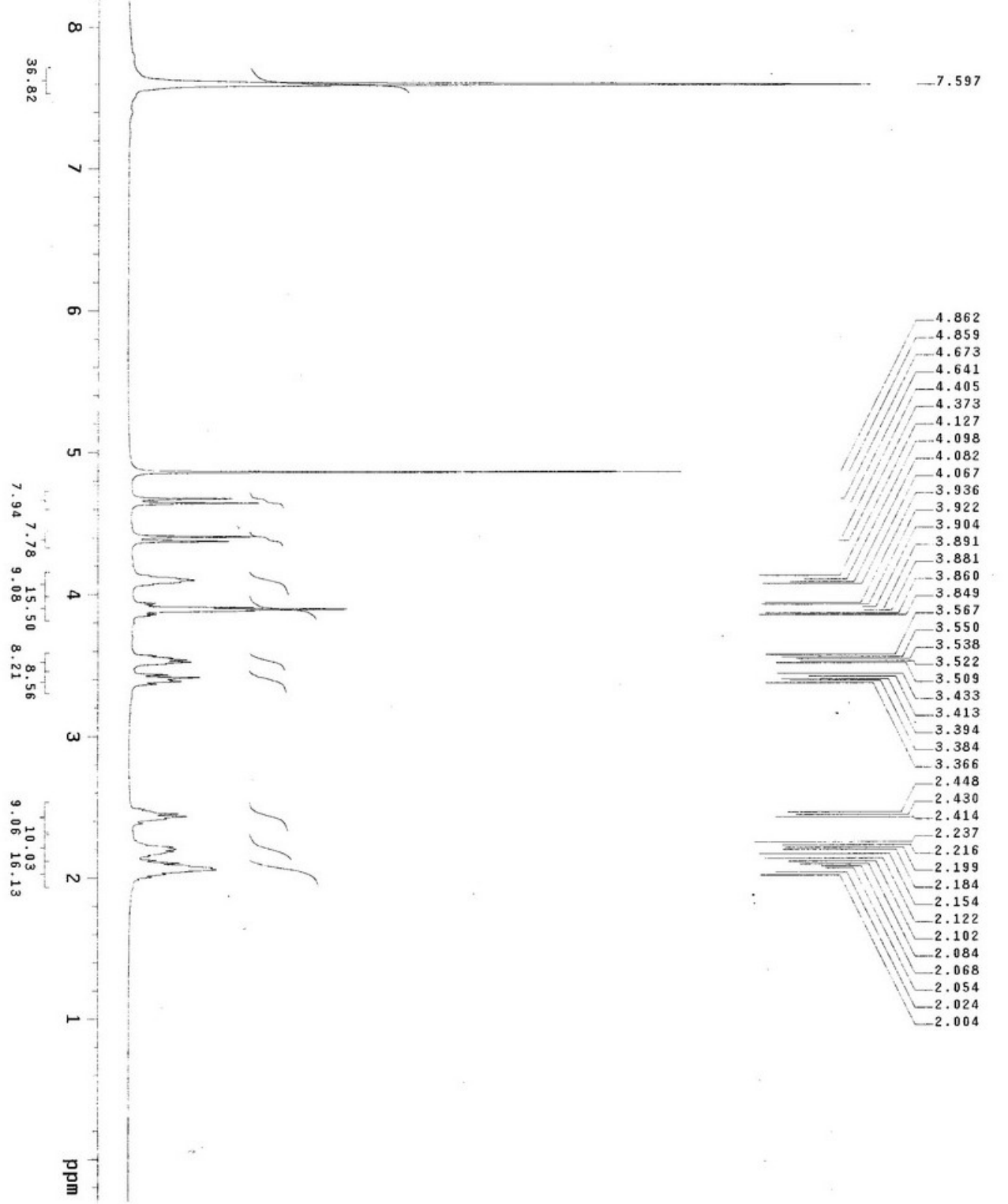



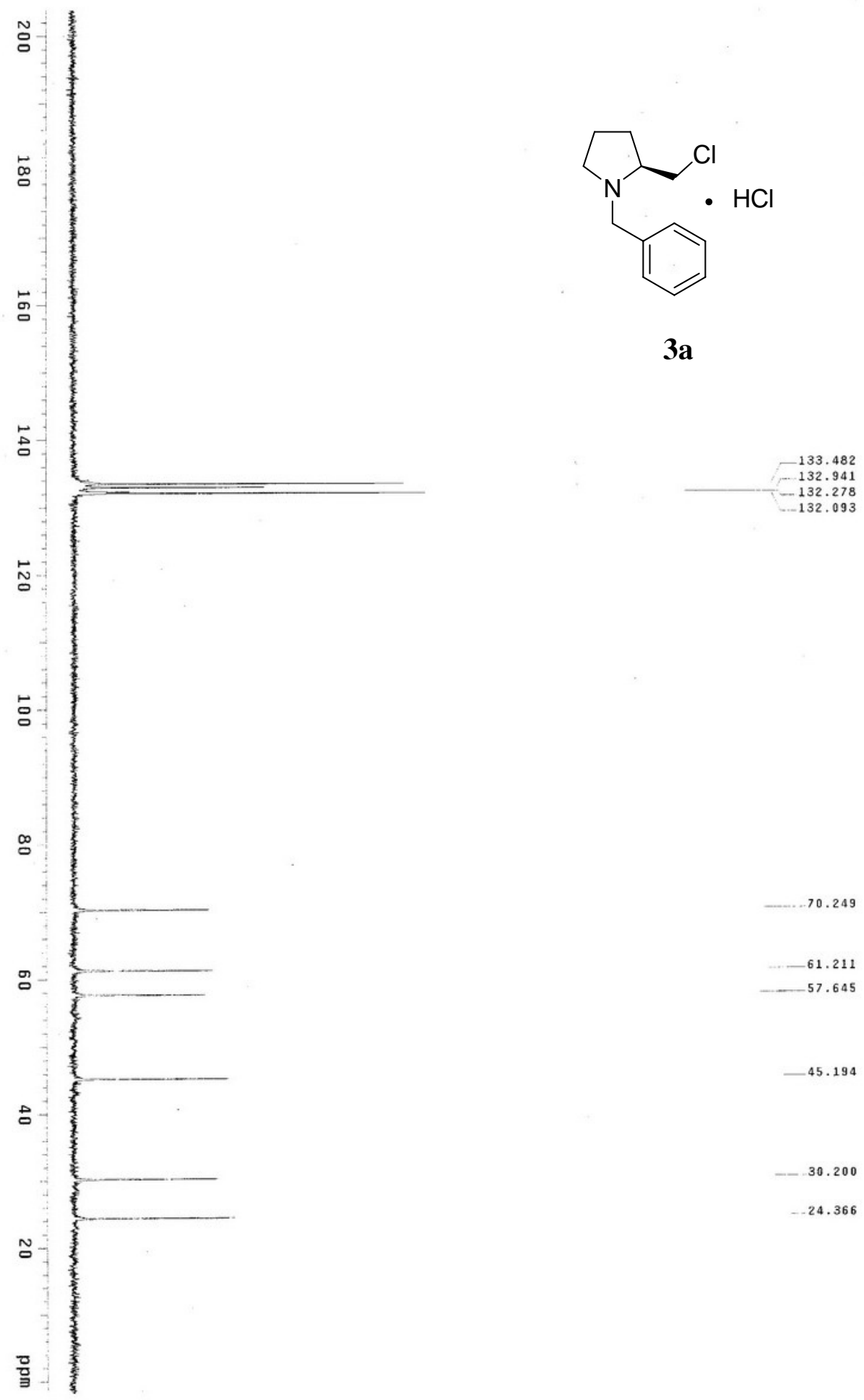

-... 61.211

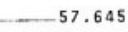

$-45.194$

$-30.200$

$-24.366$ 


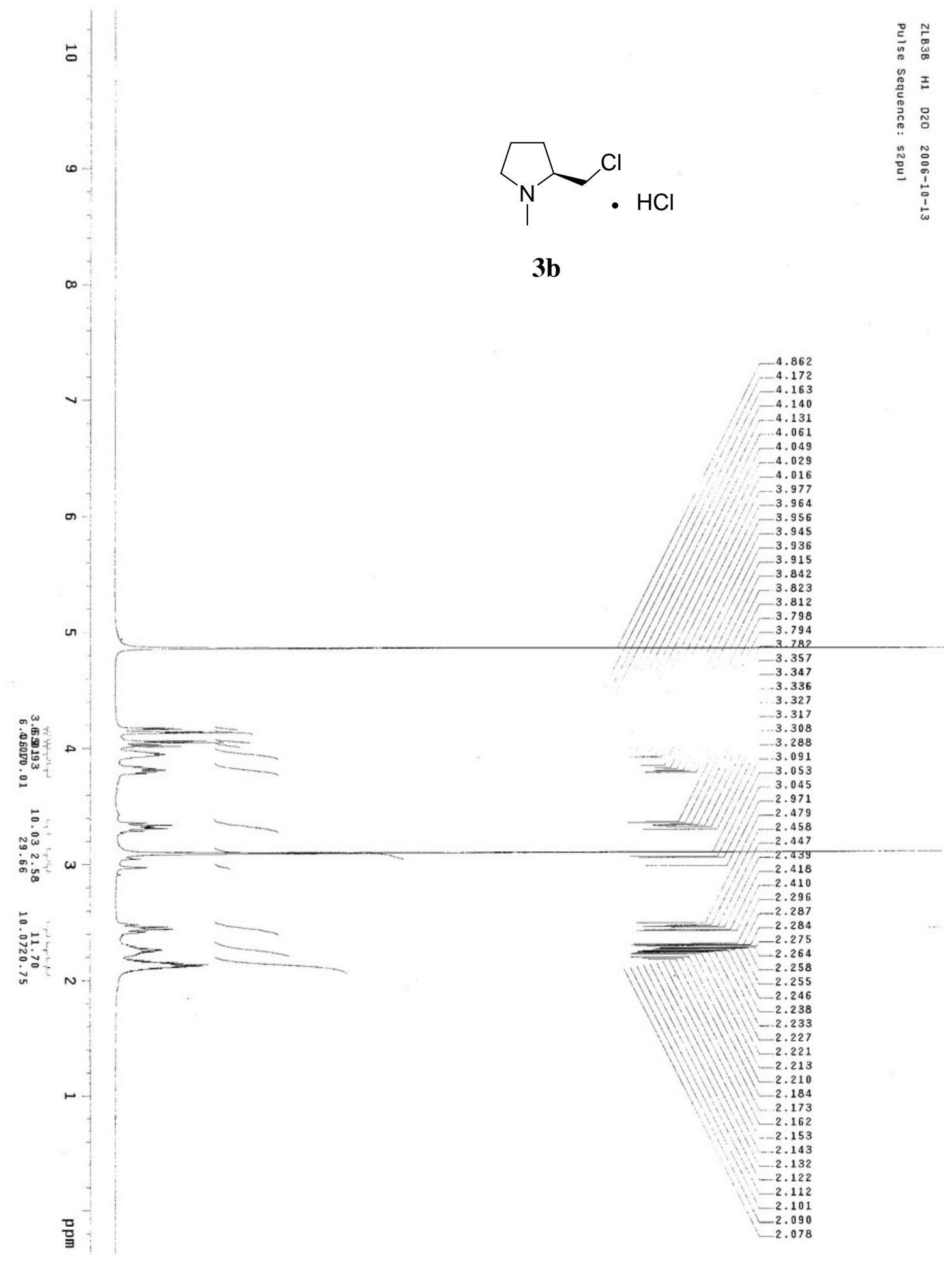



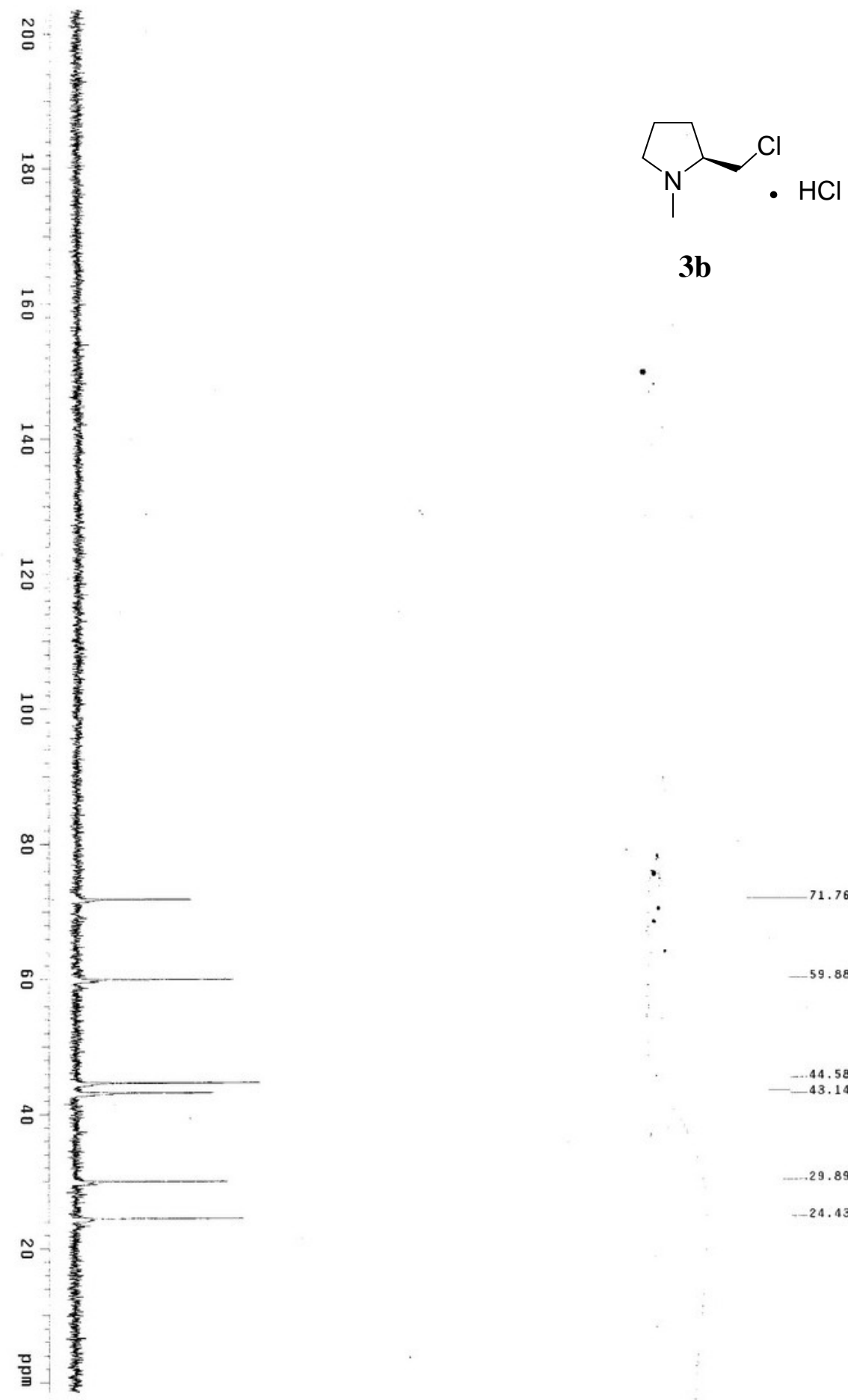

3b

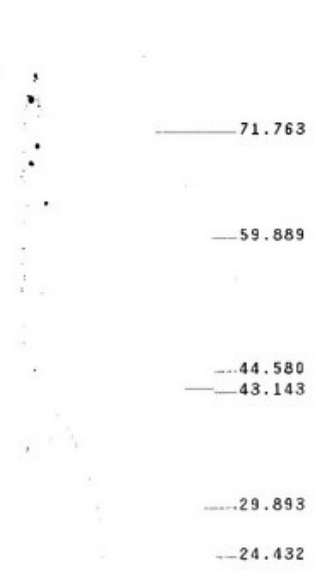




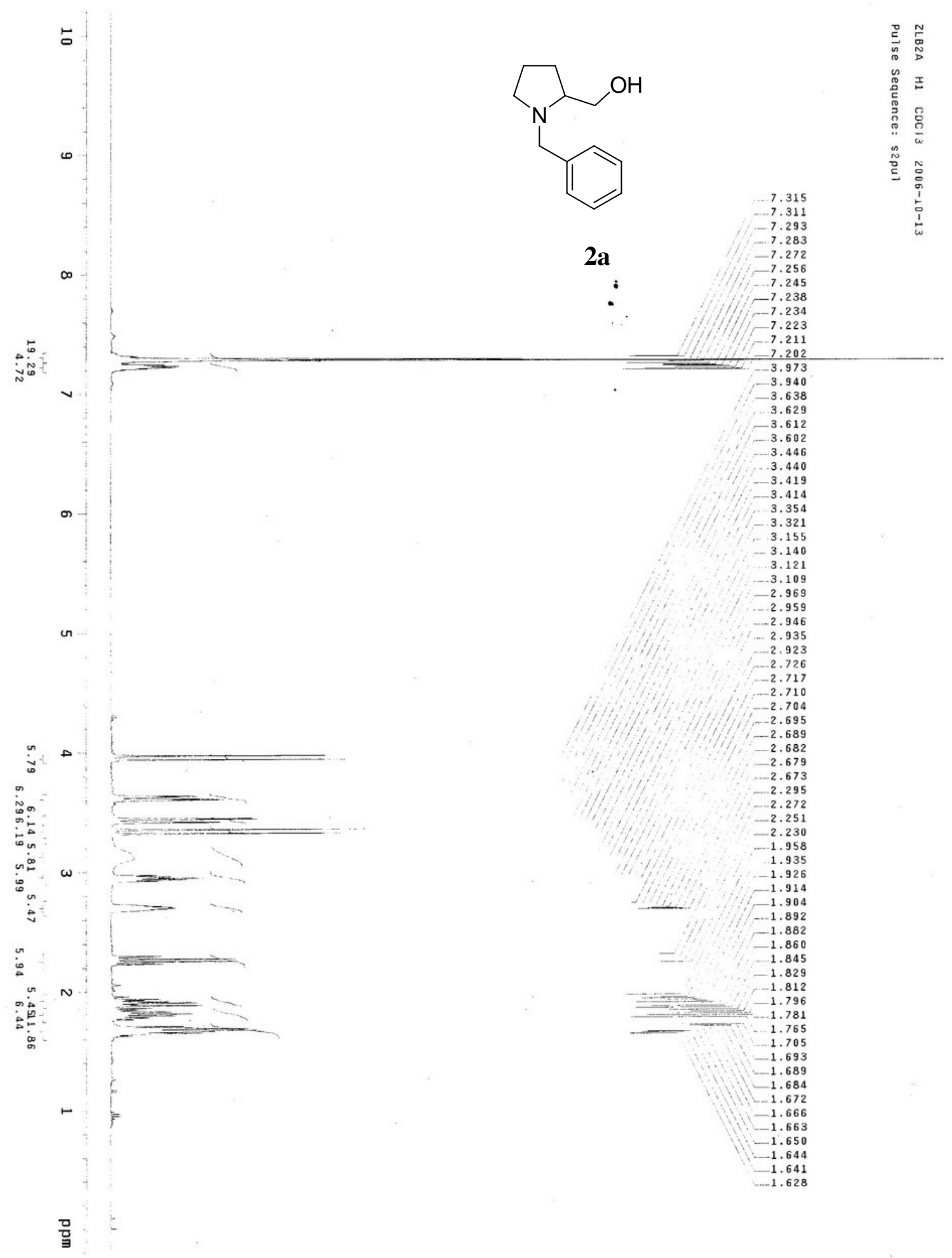



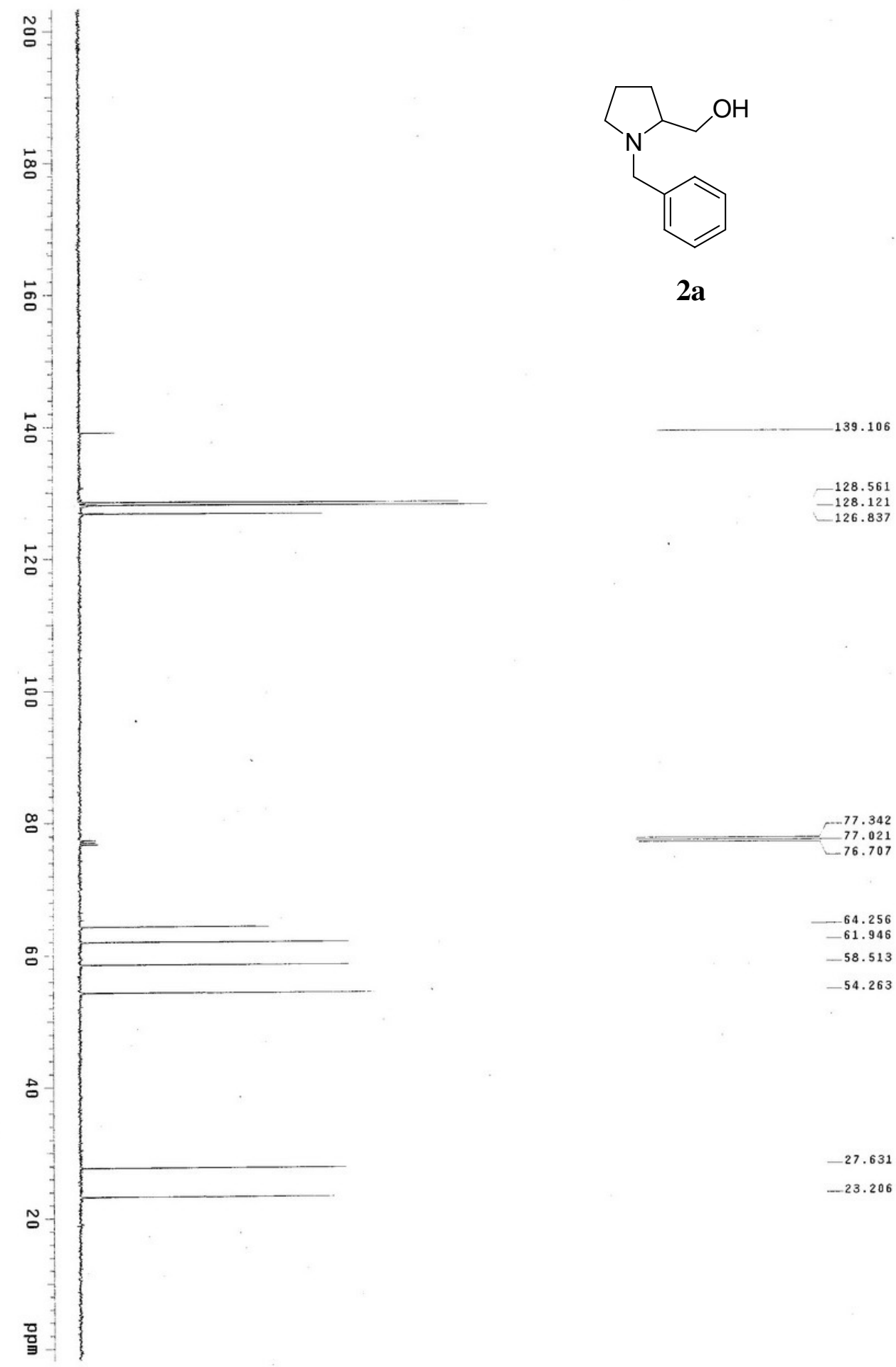

$-58.513$

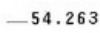

$-27.631$

$-23.206$ 


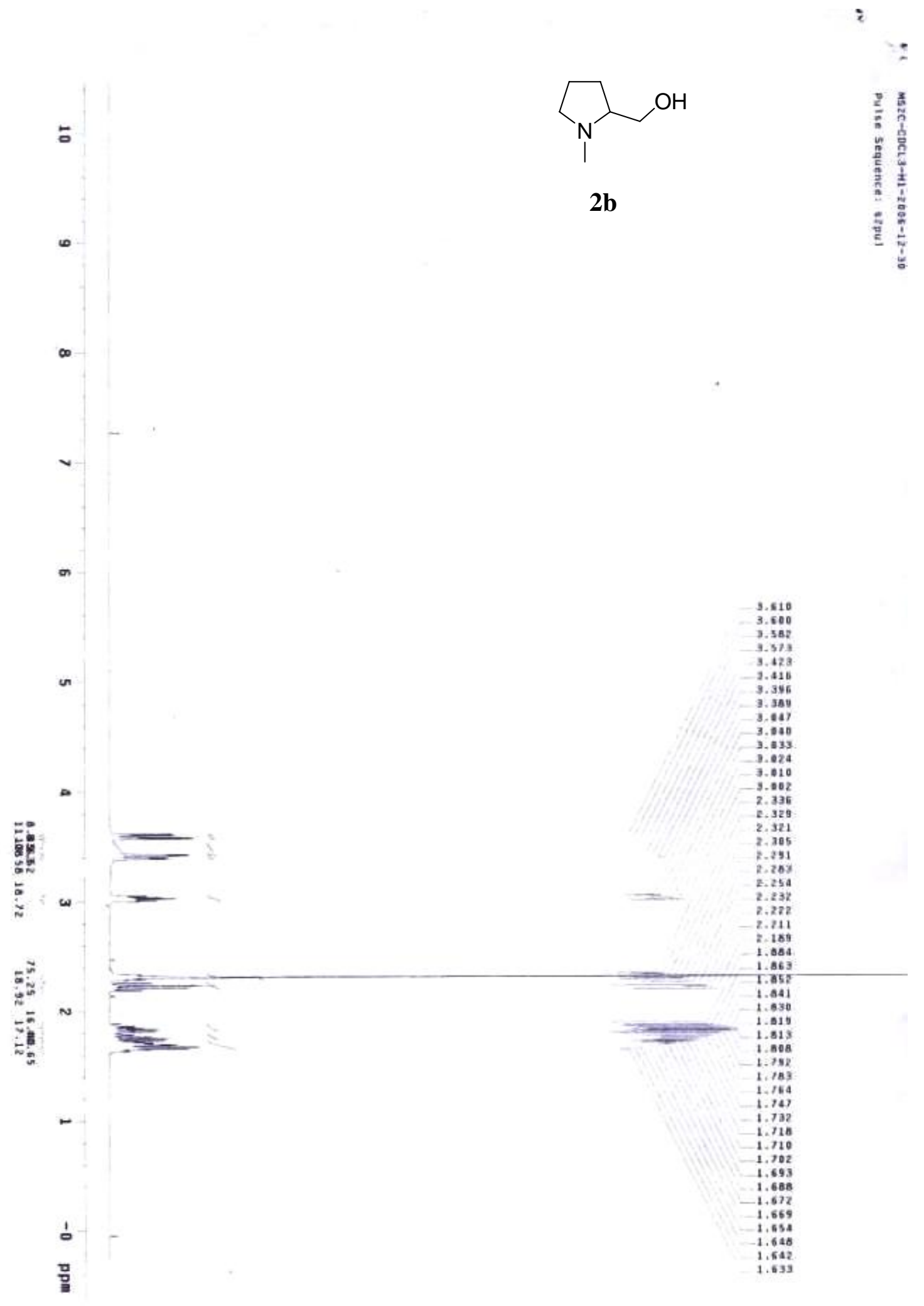




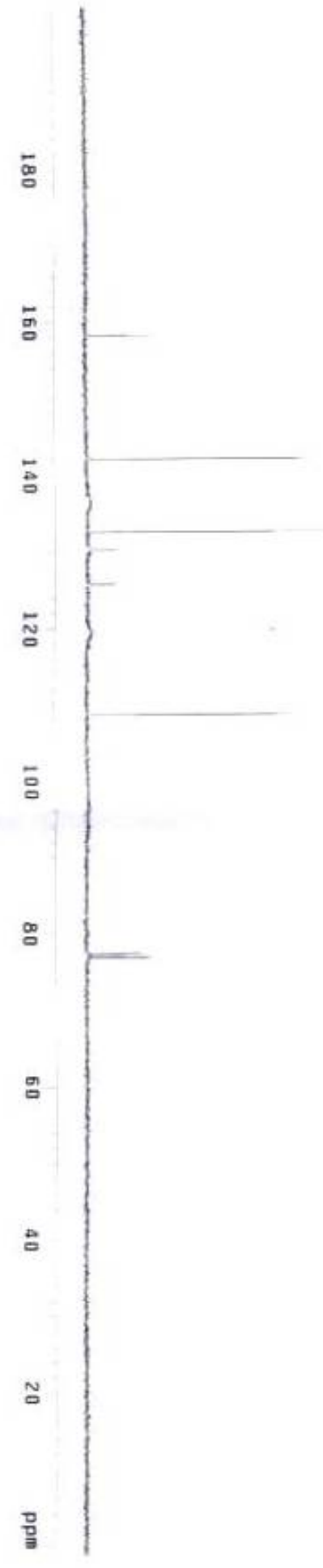

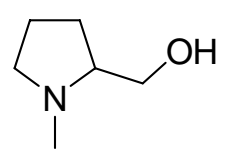

2b

$-158.235$

142.079

132.511
130,263

125. 734

10n. 19

17. 347

$71+177$
$76+126$

76. 395 


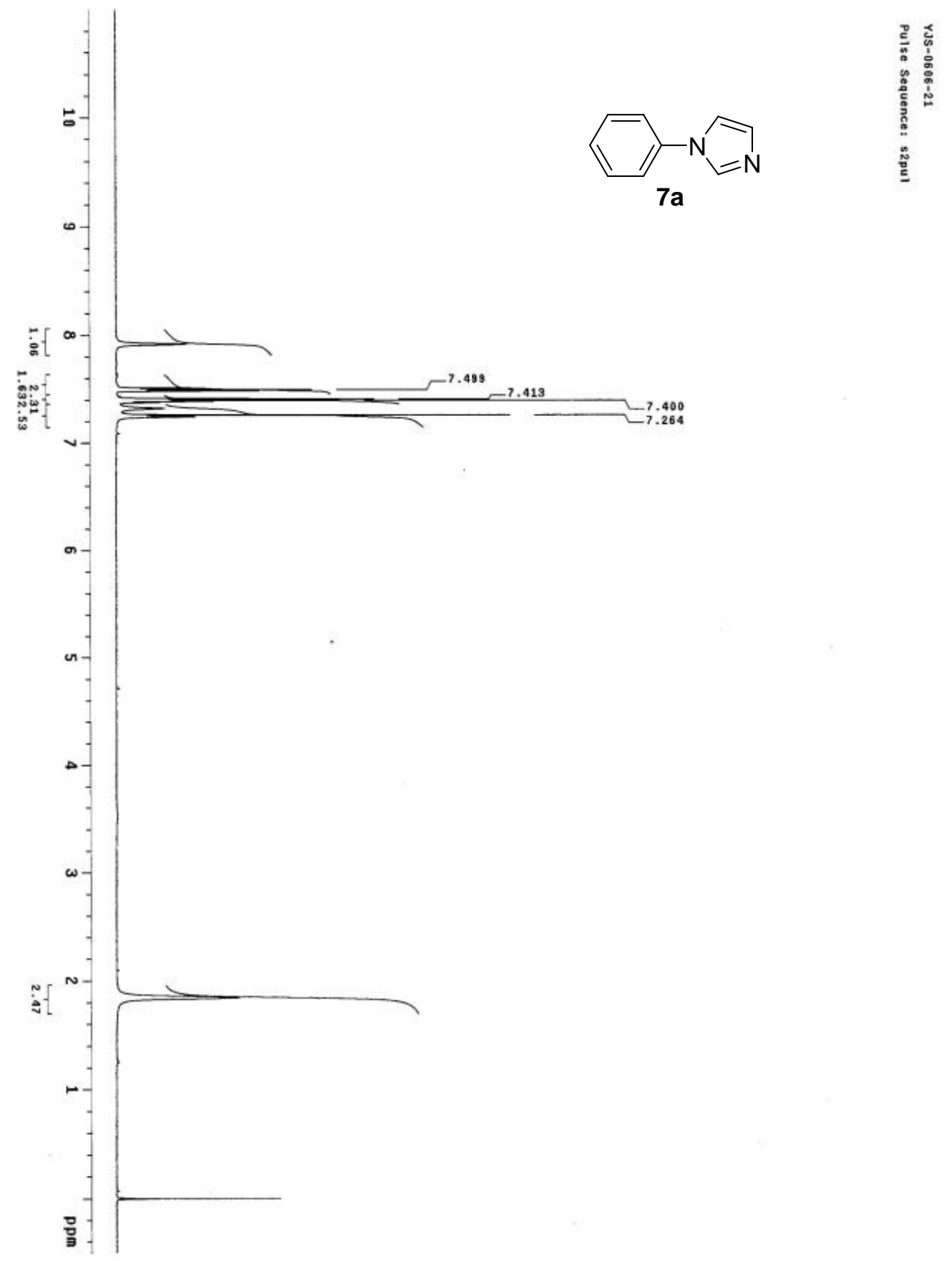




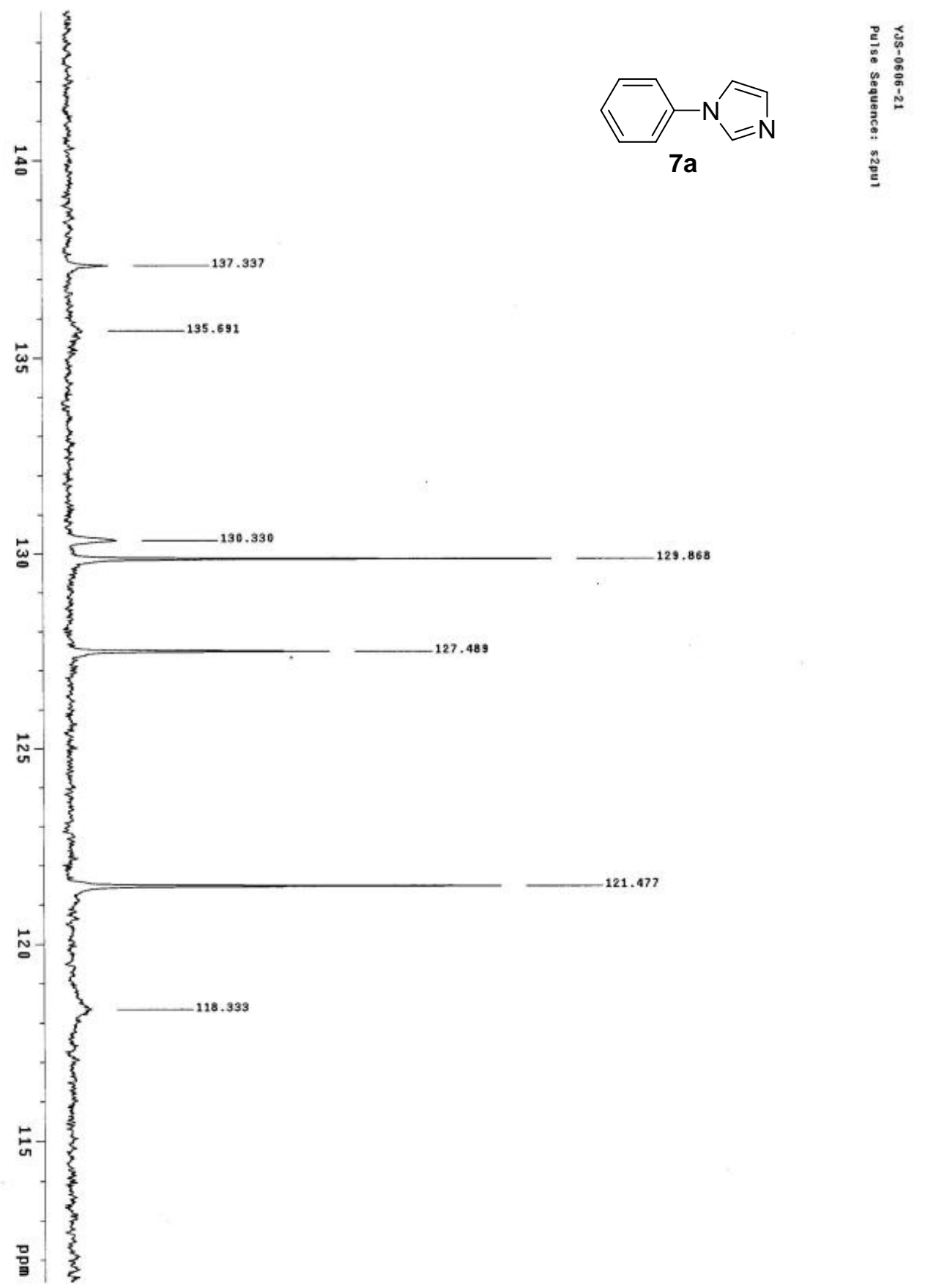




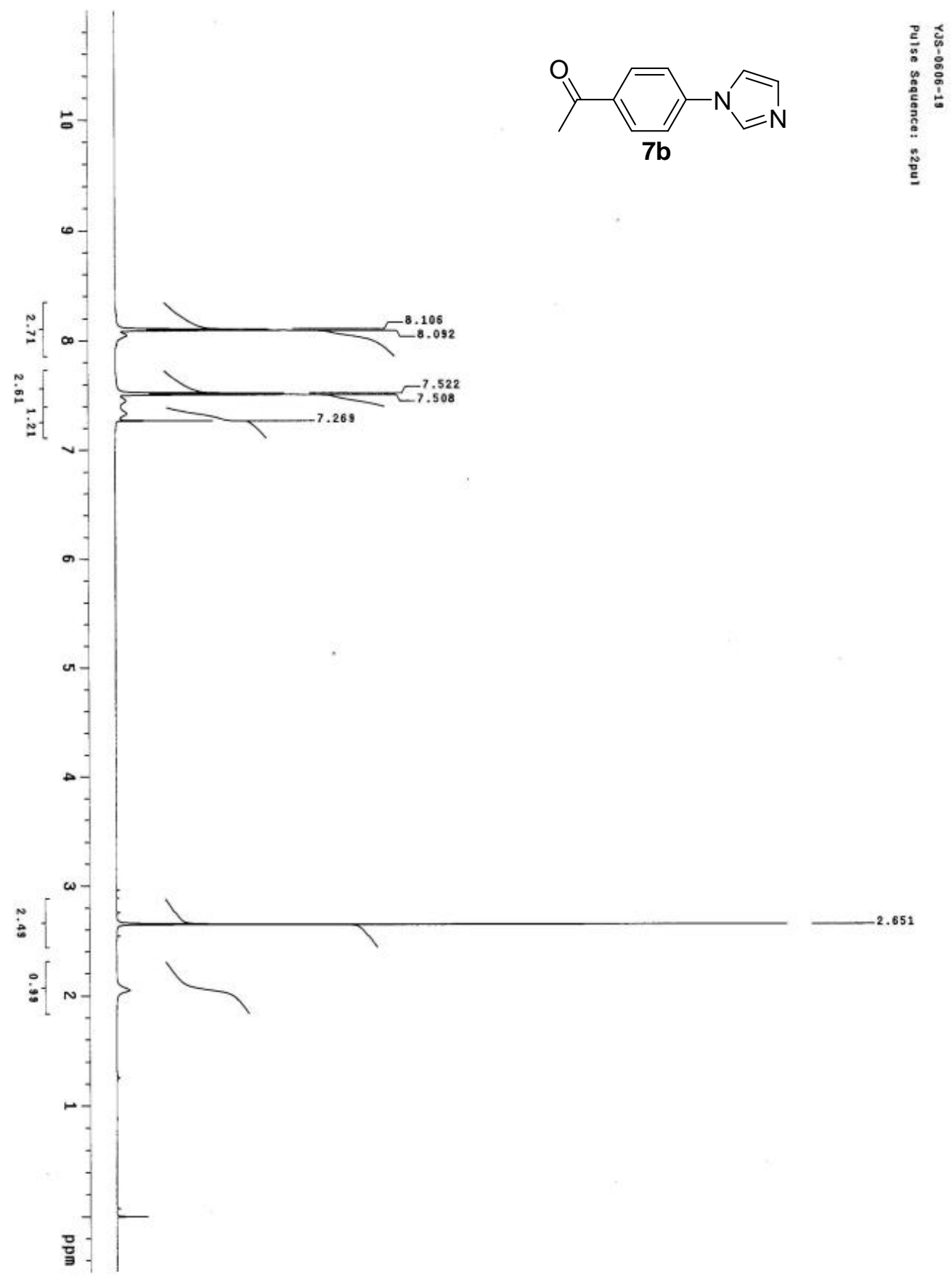




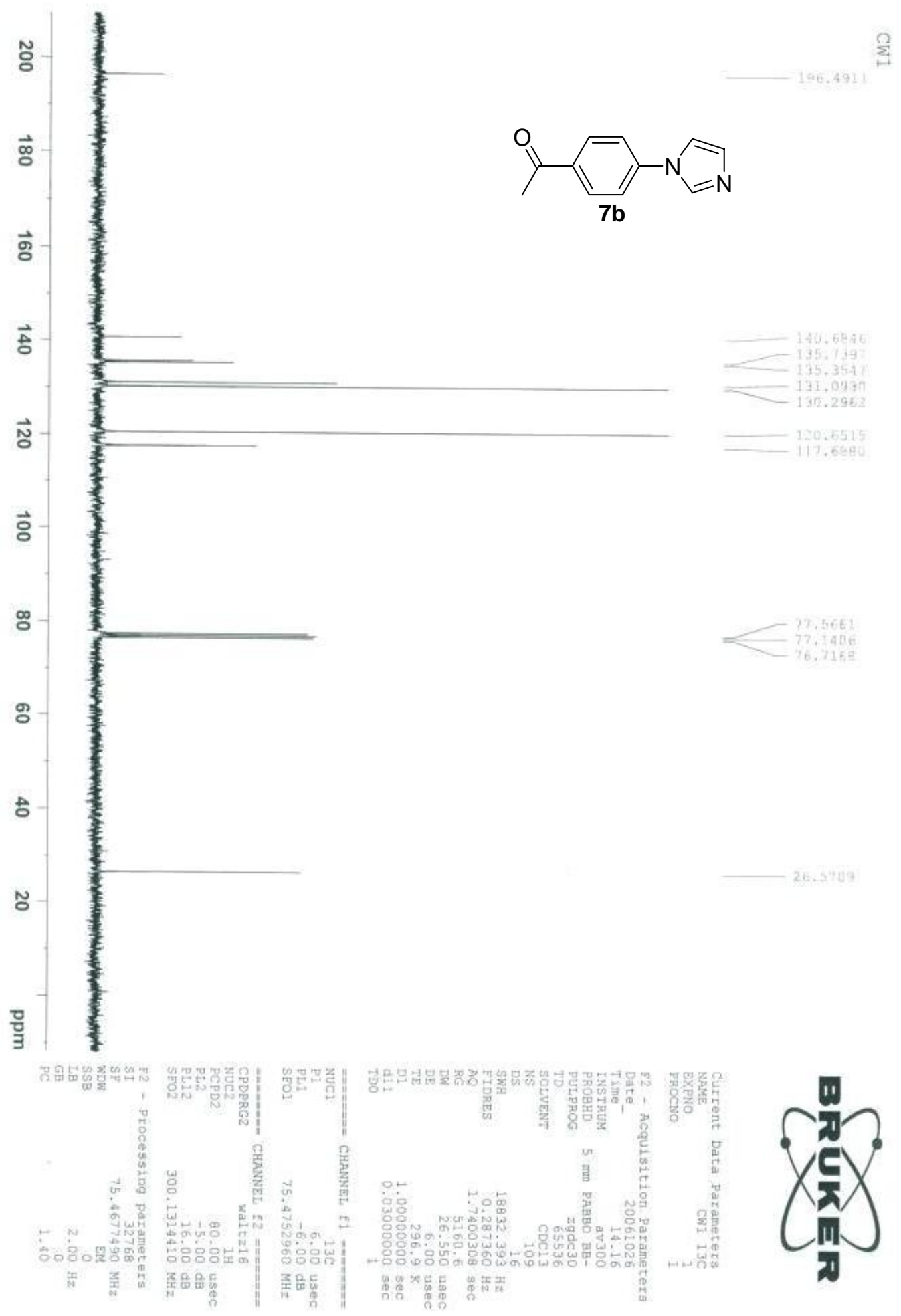



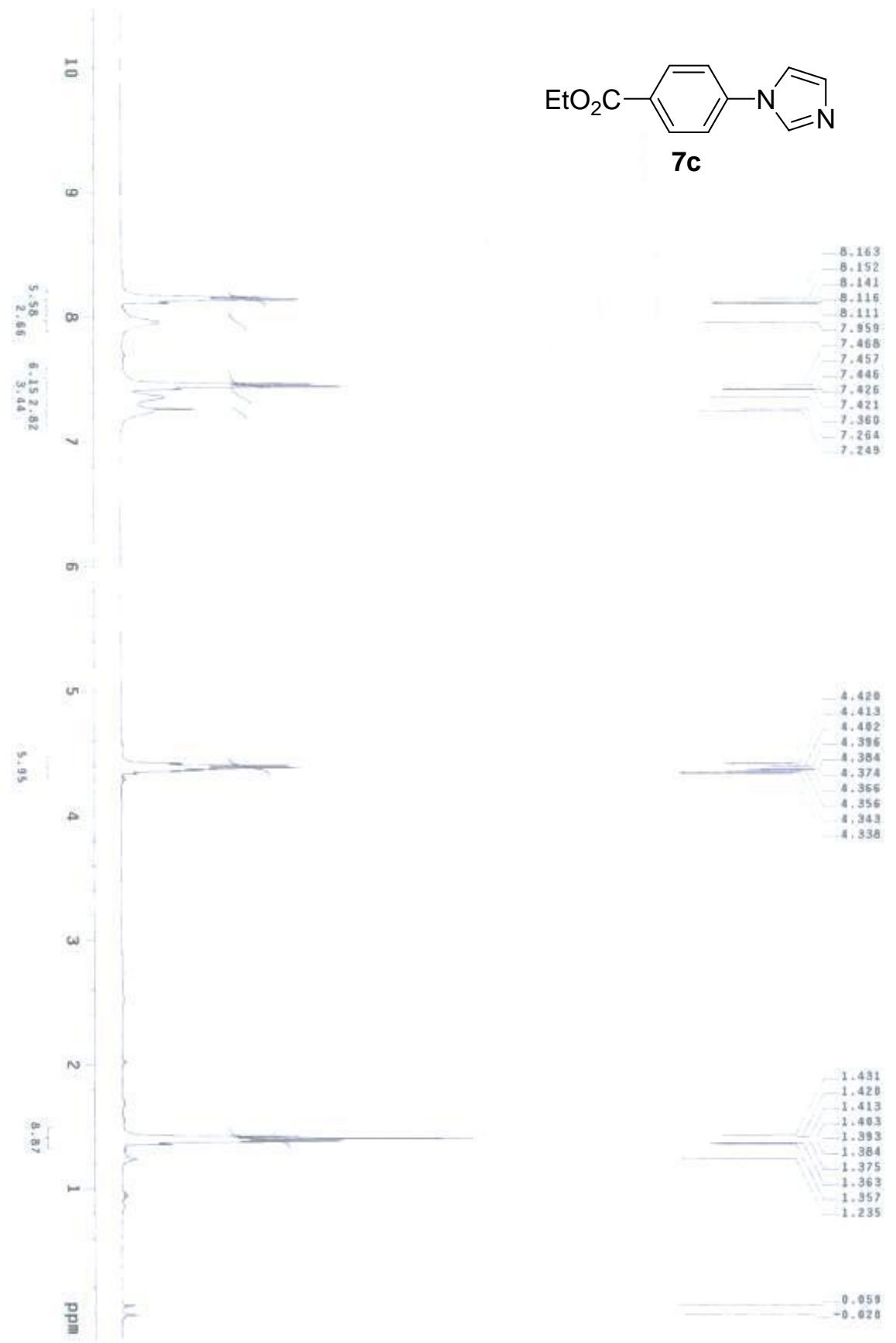


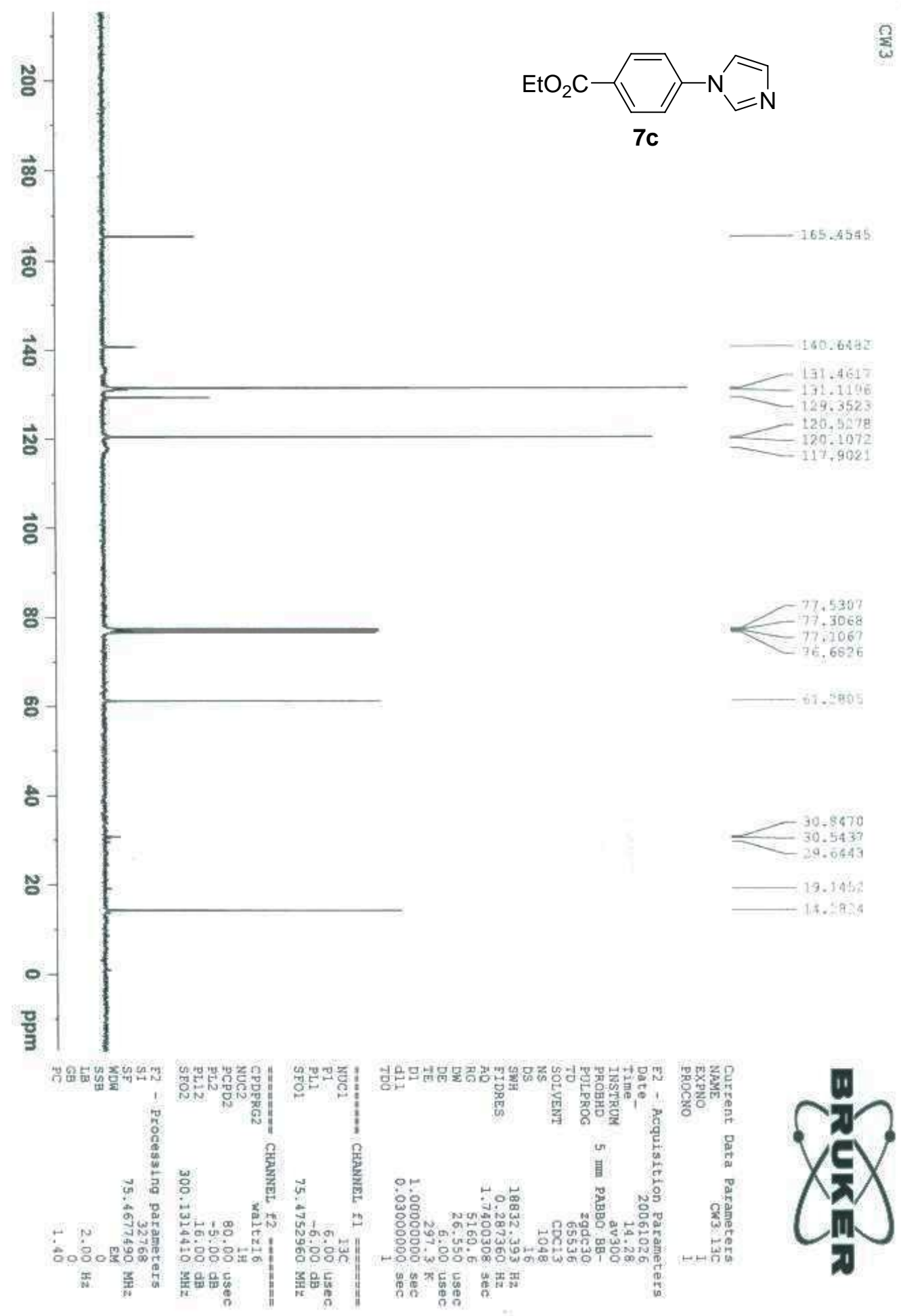




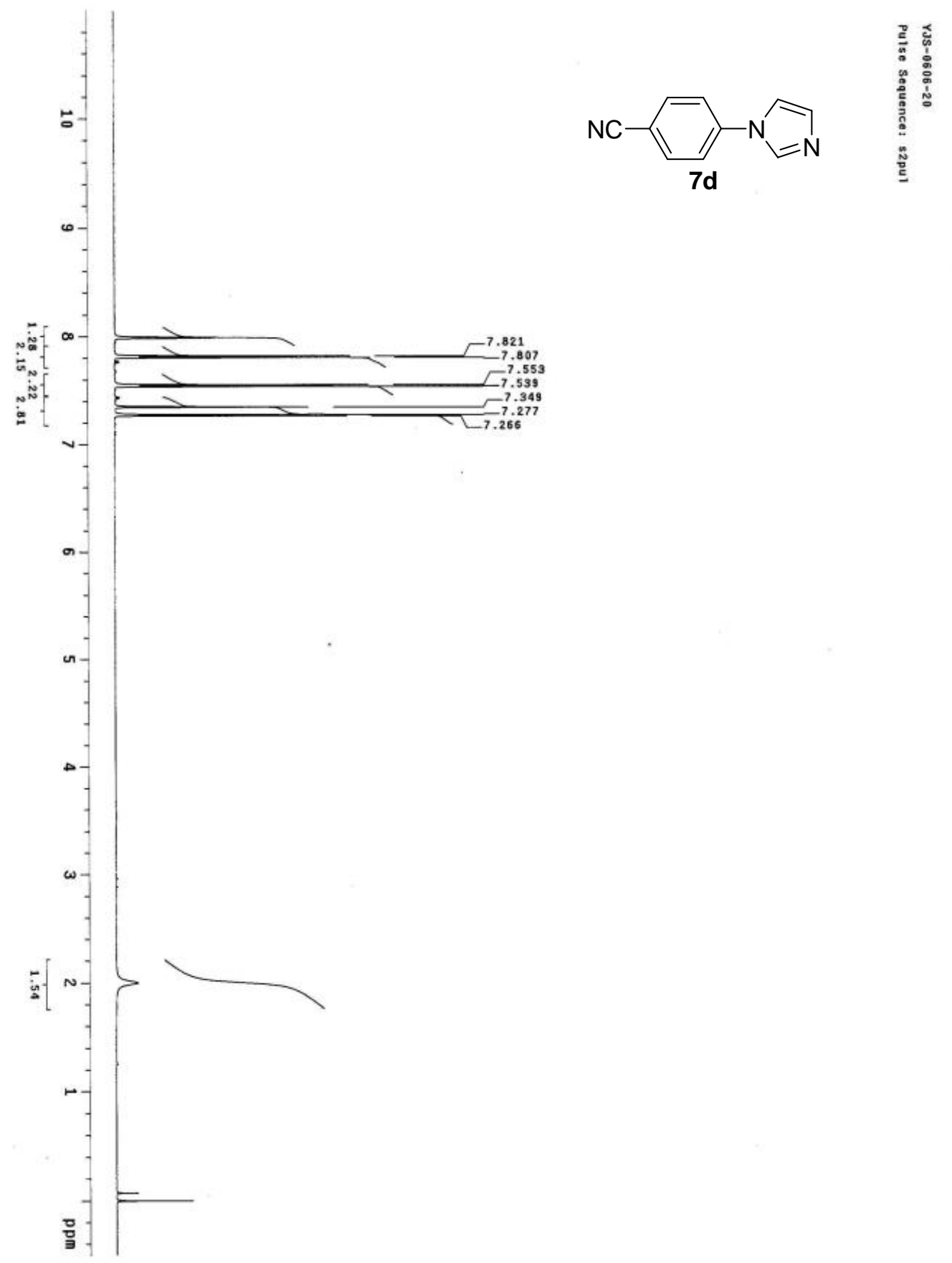




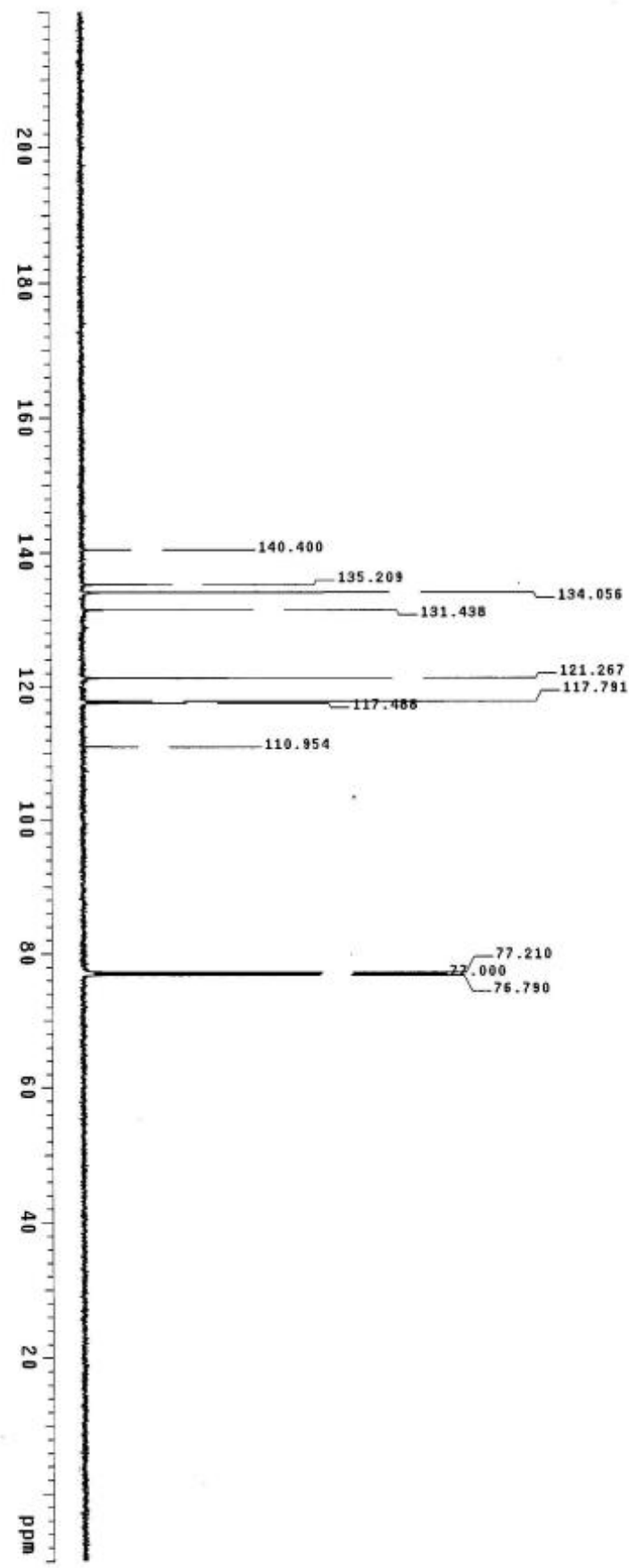




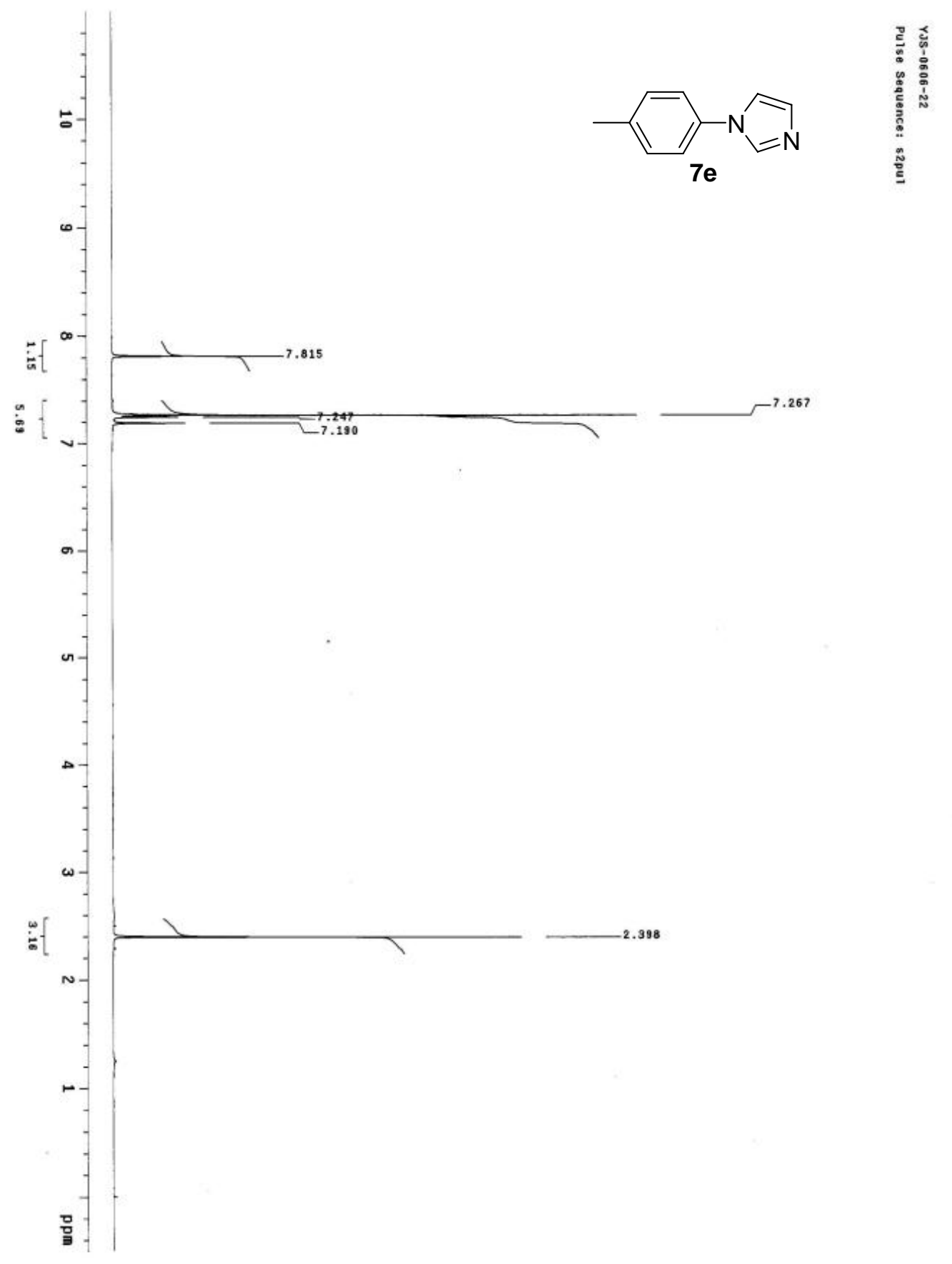



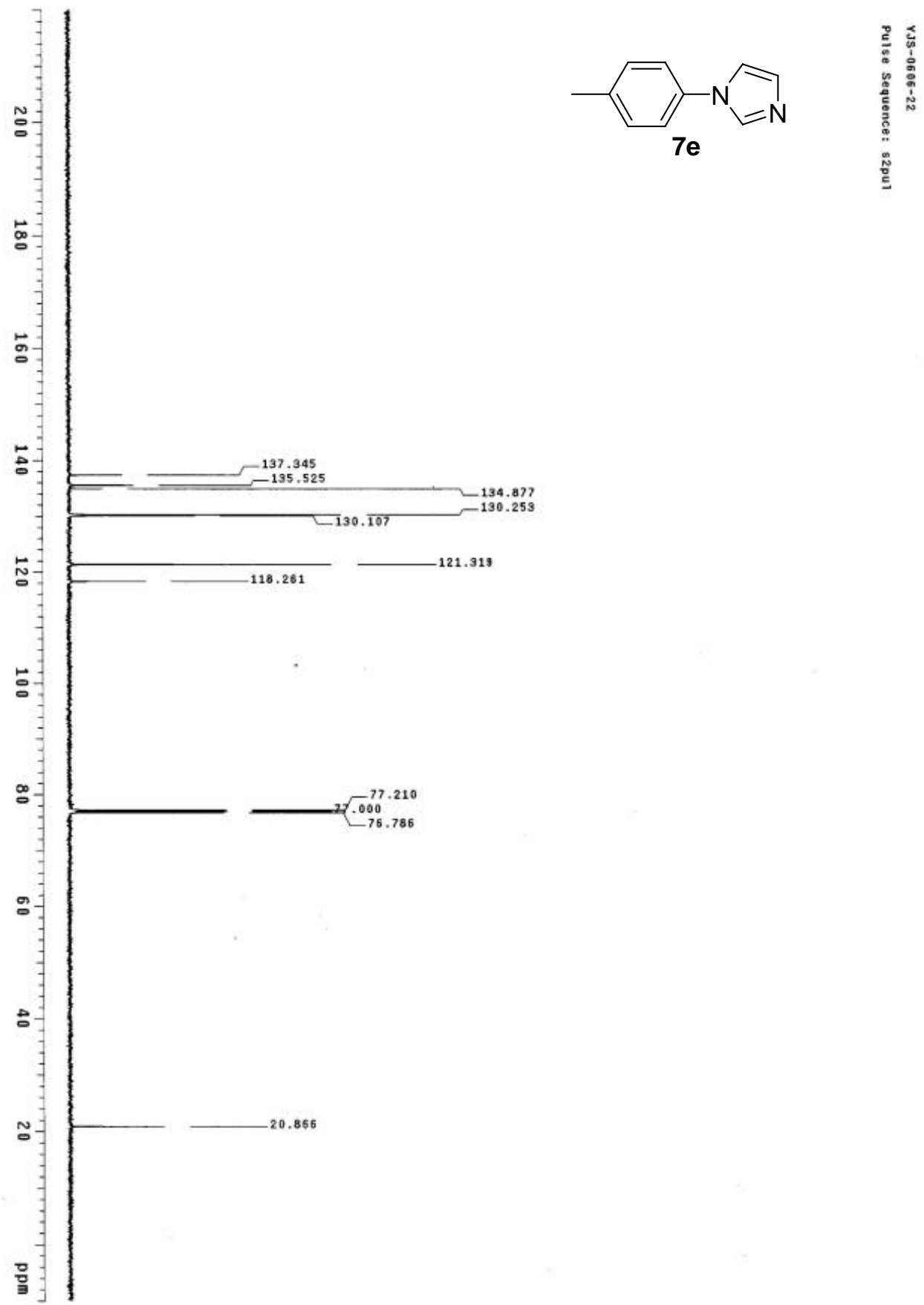


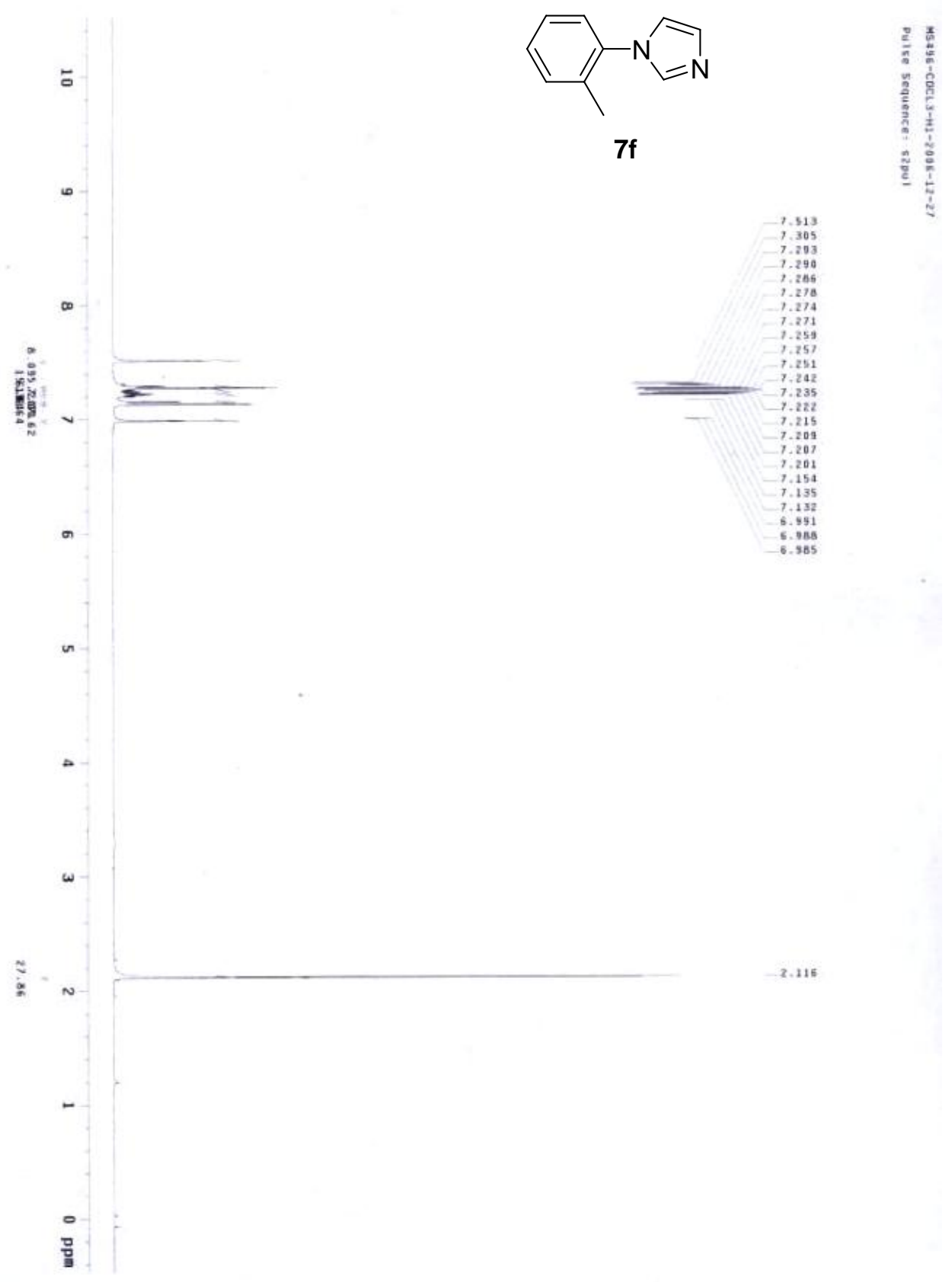



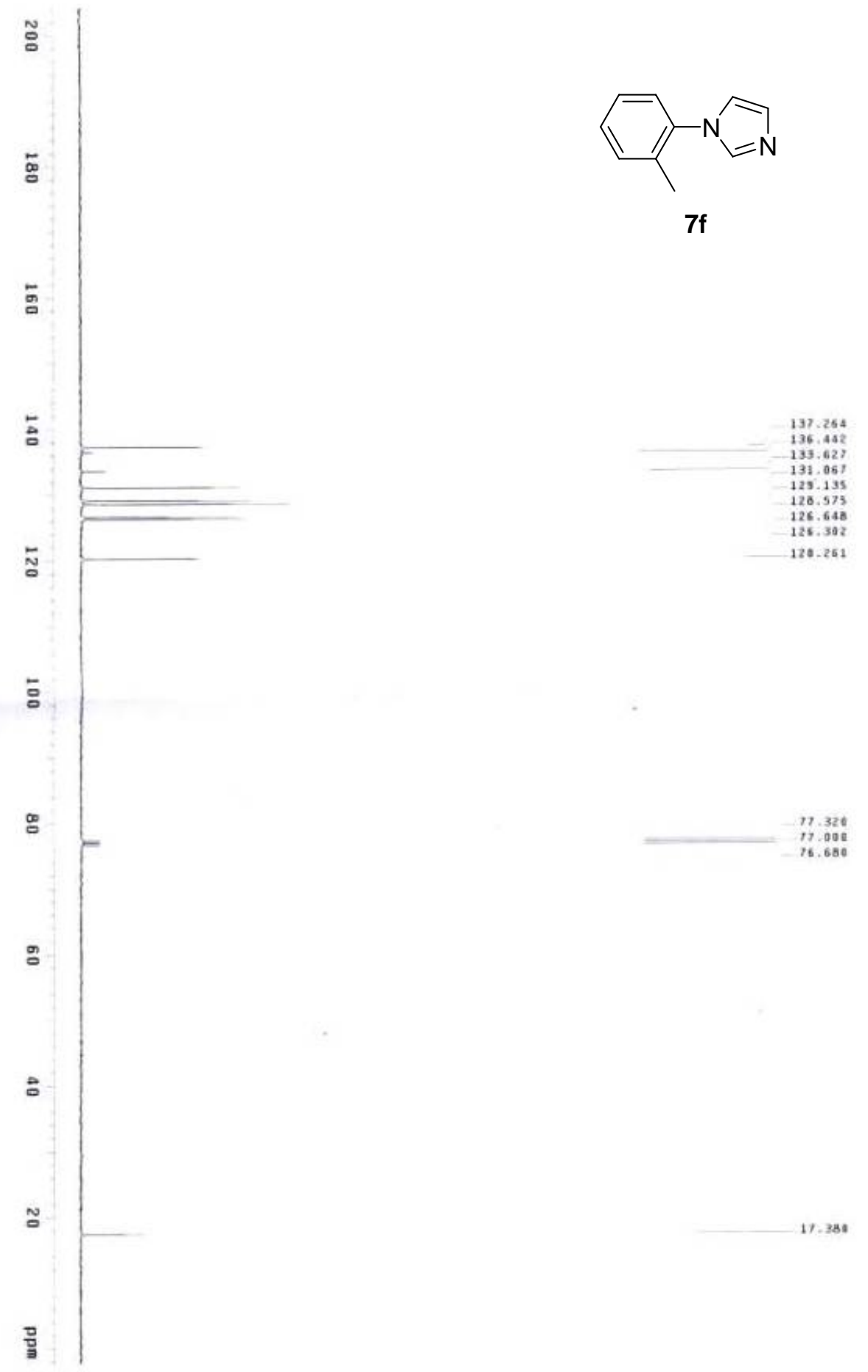

77.326
77.001

77. 7000

17. 349 


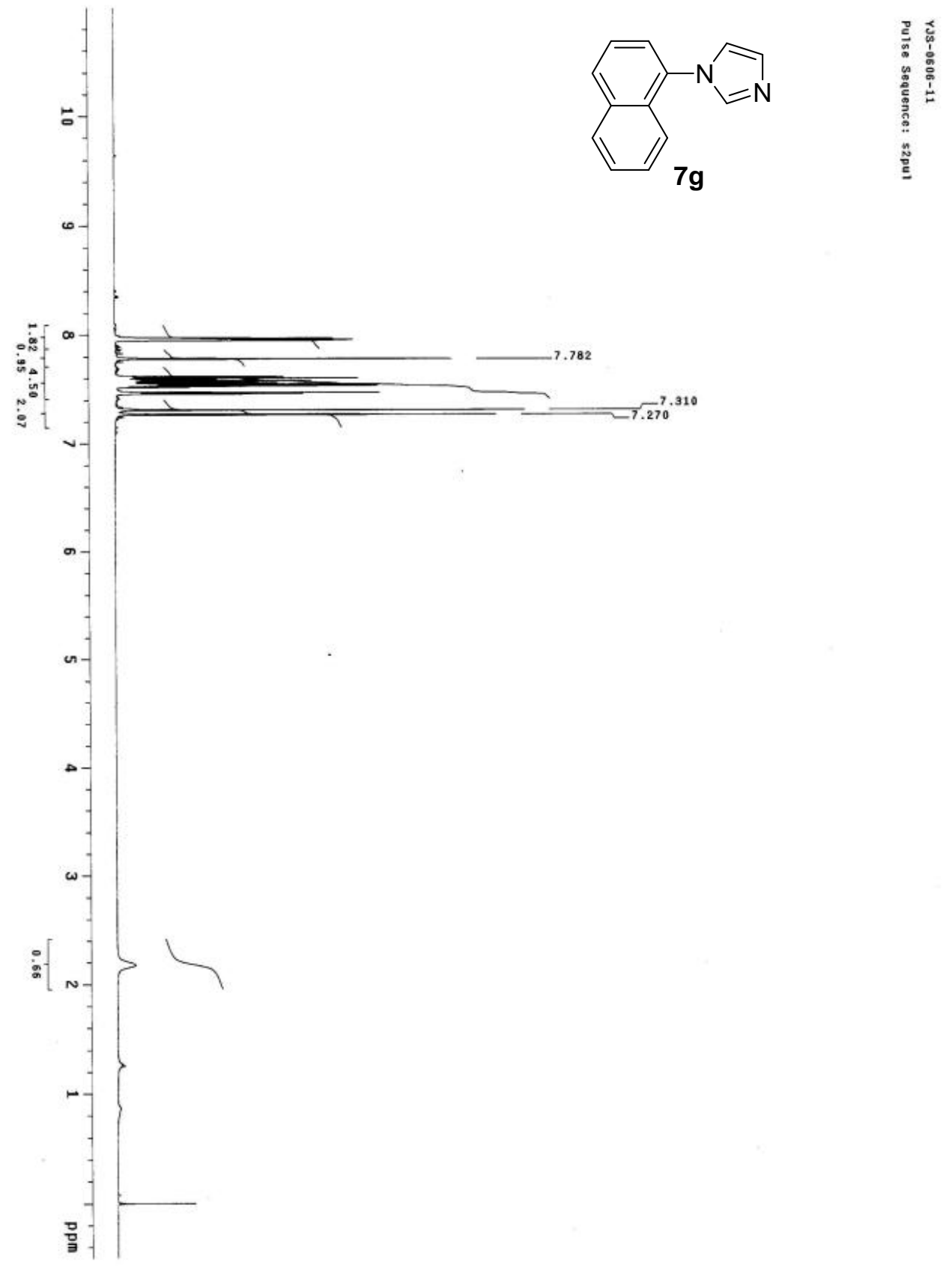



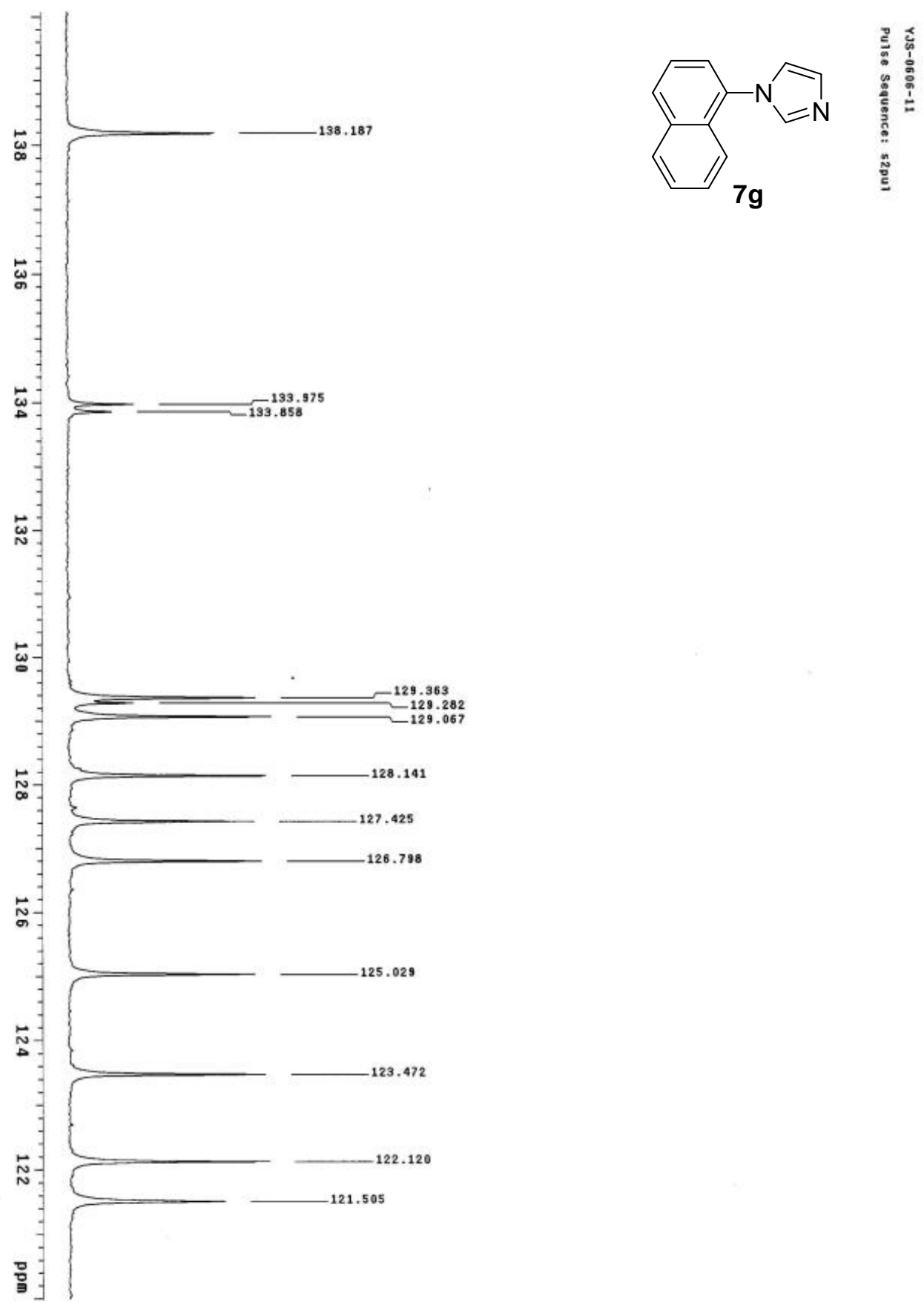


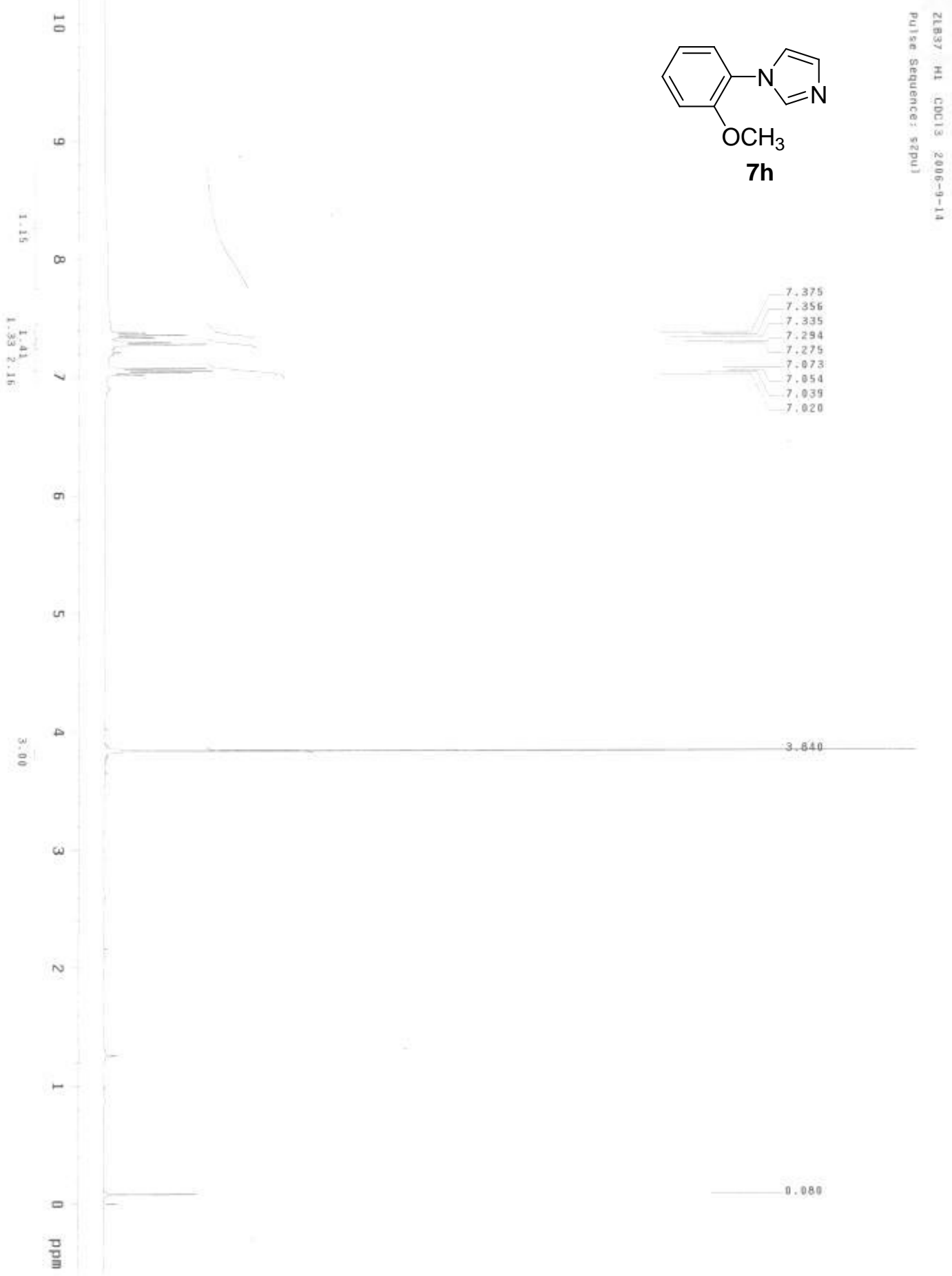



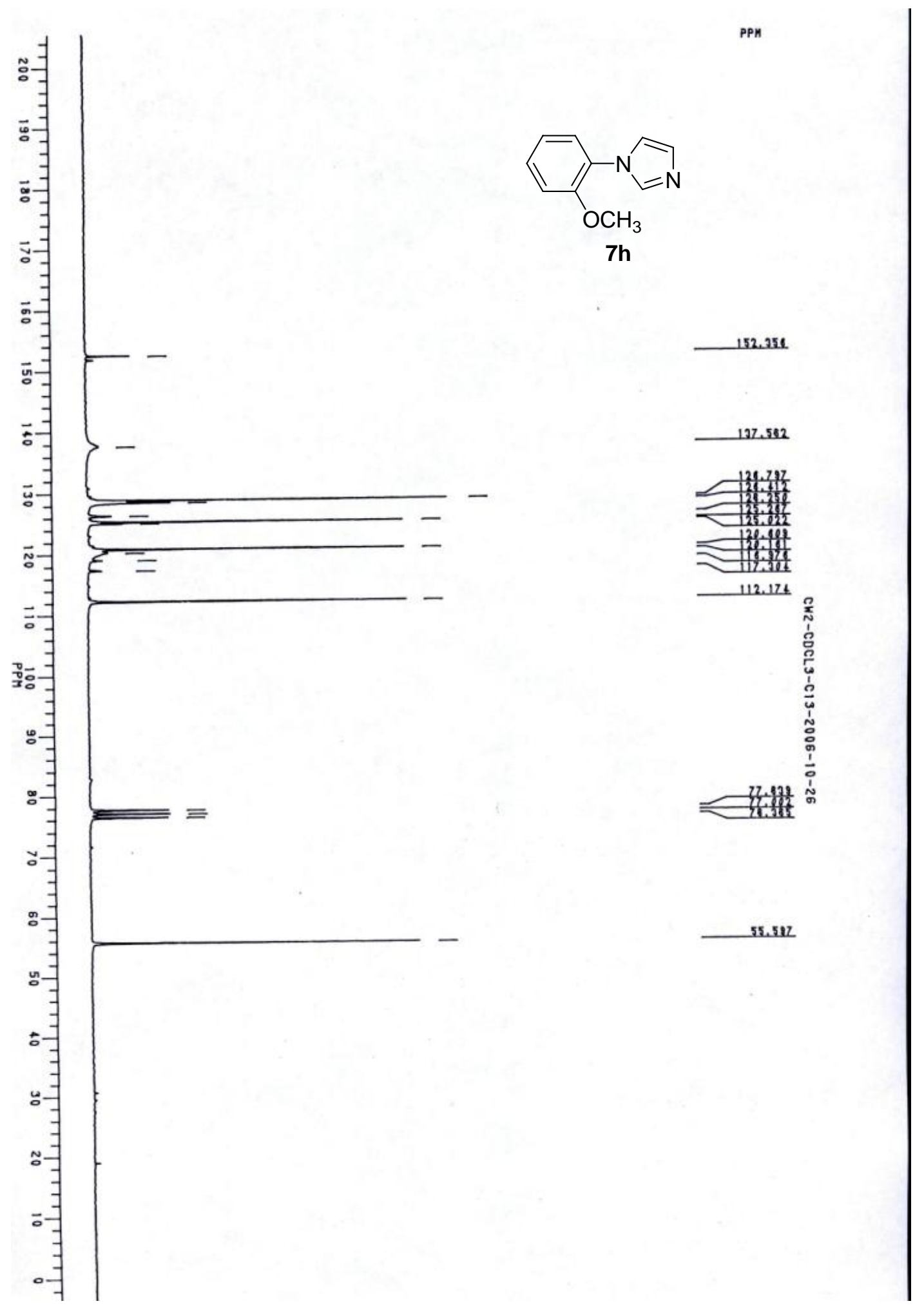


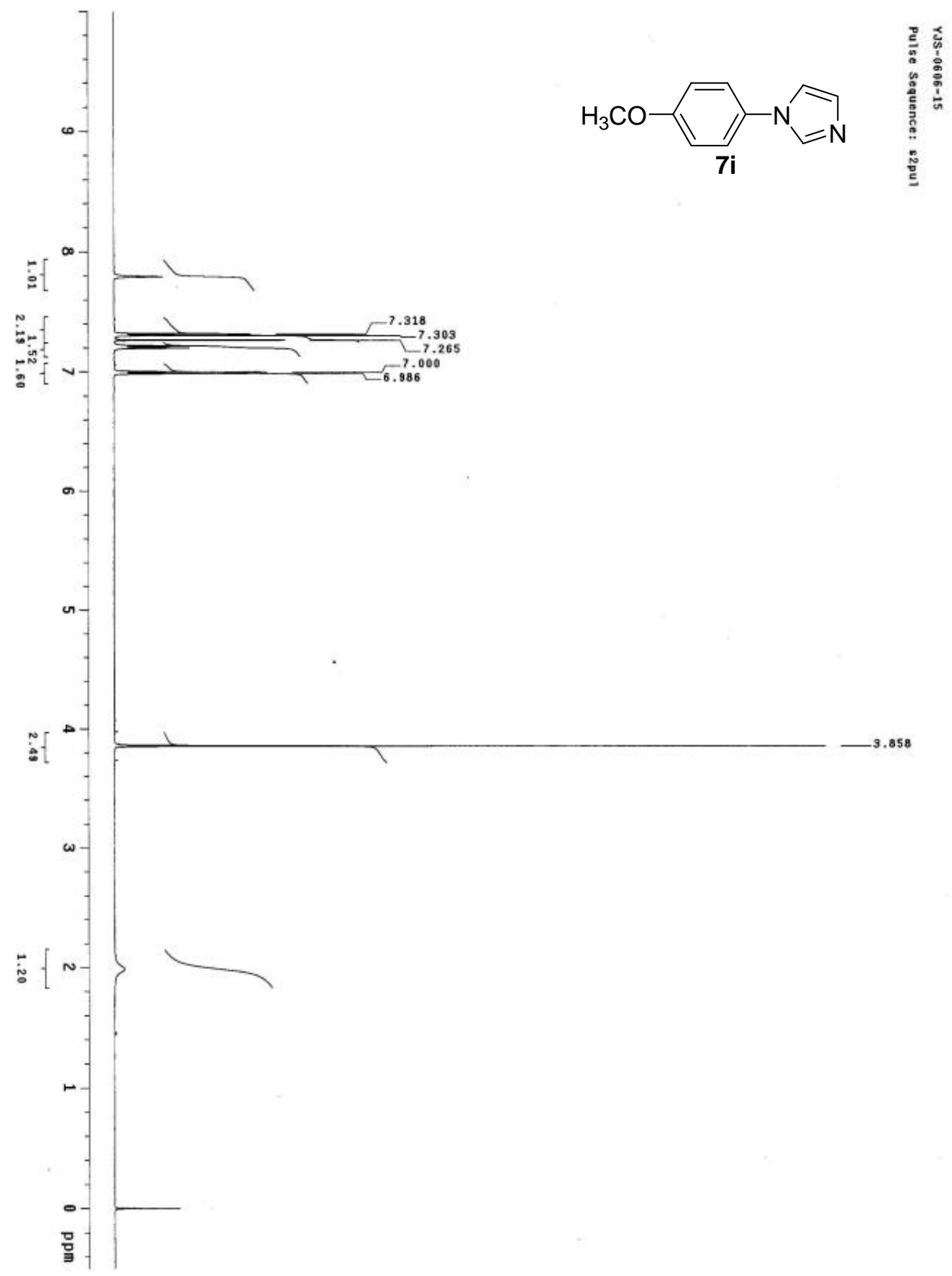




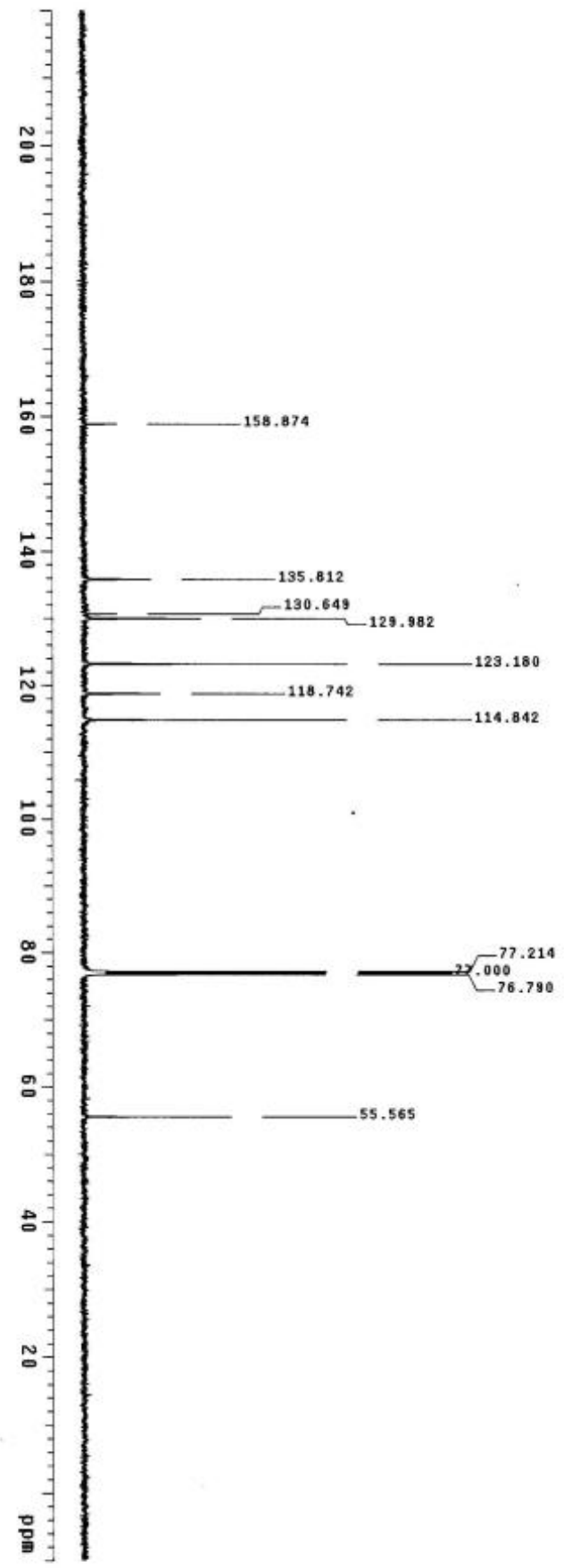




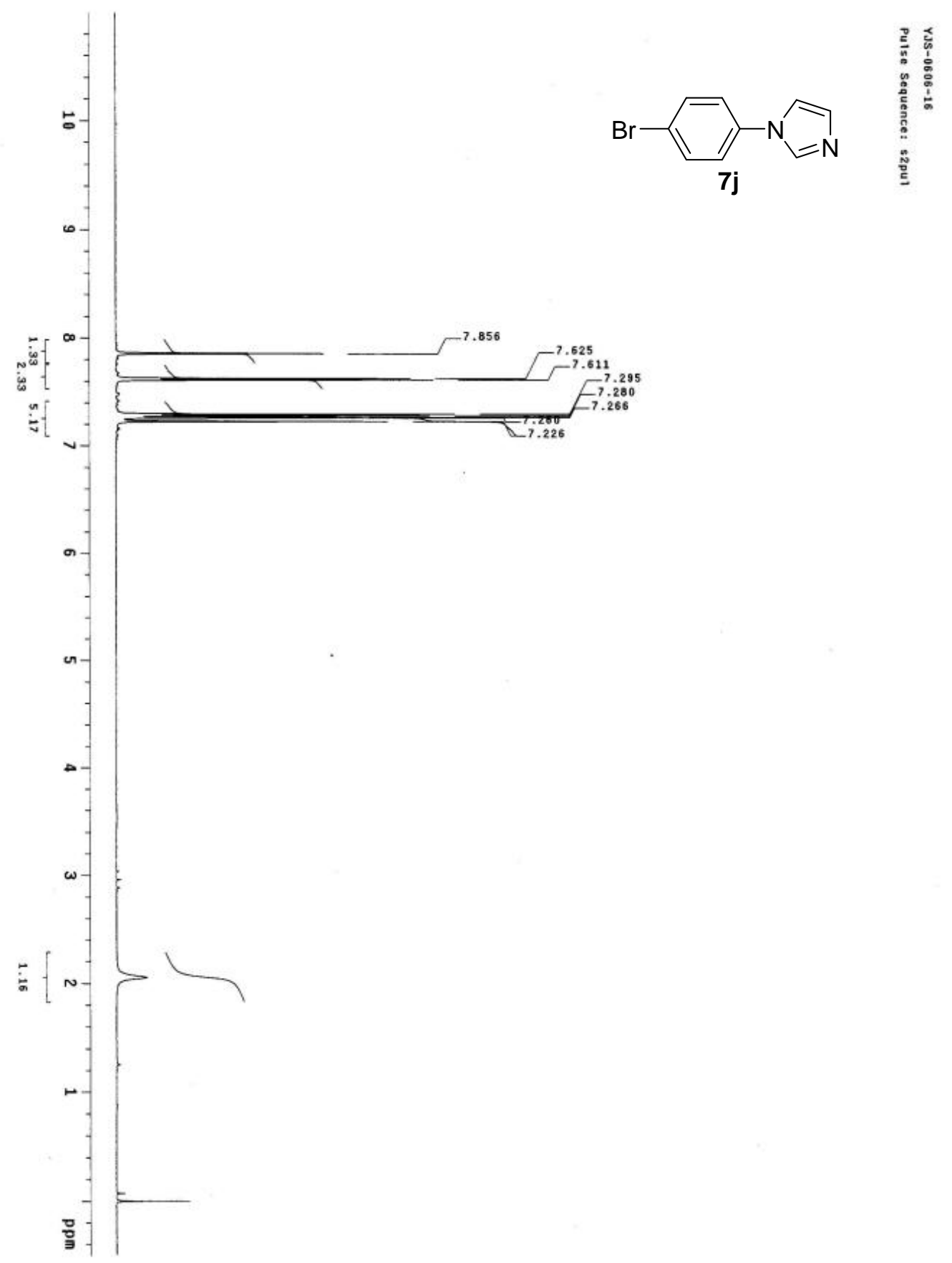



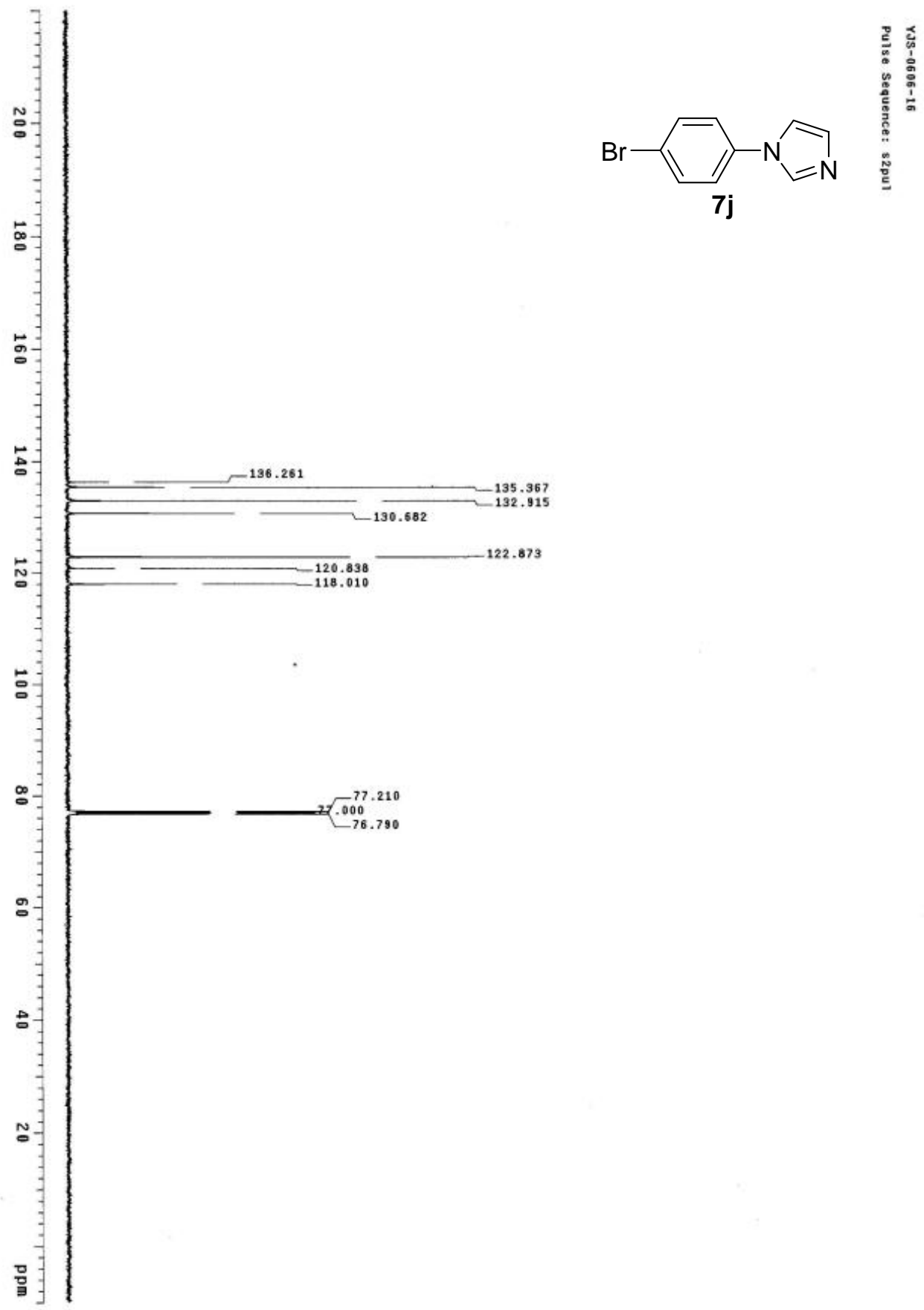


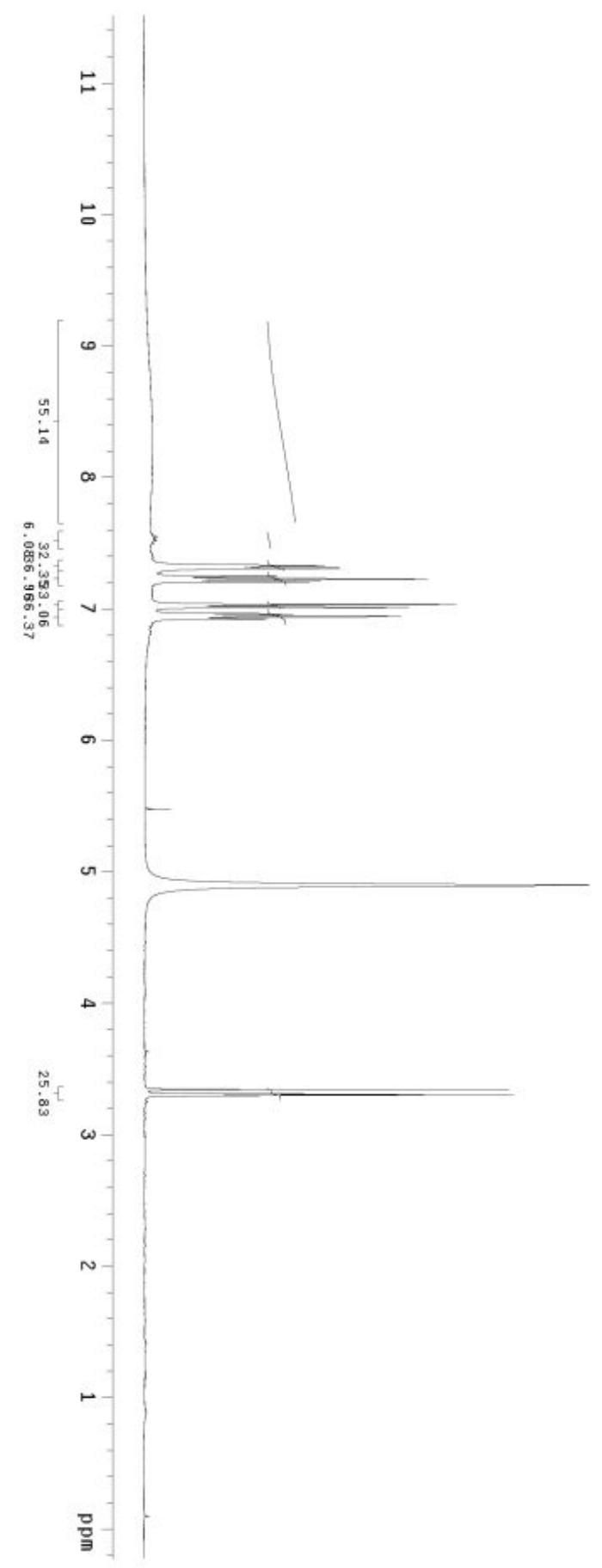

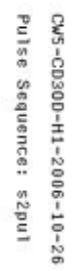
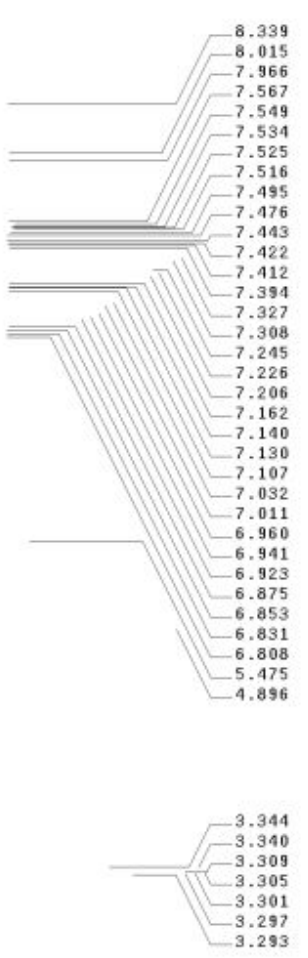


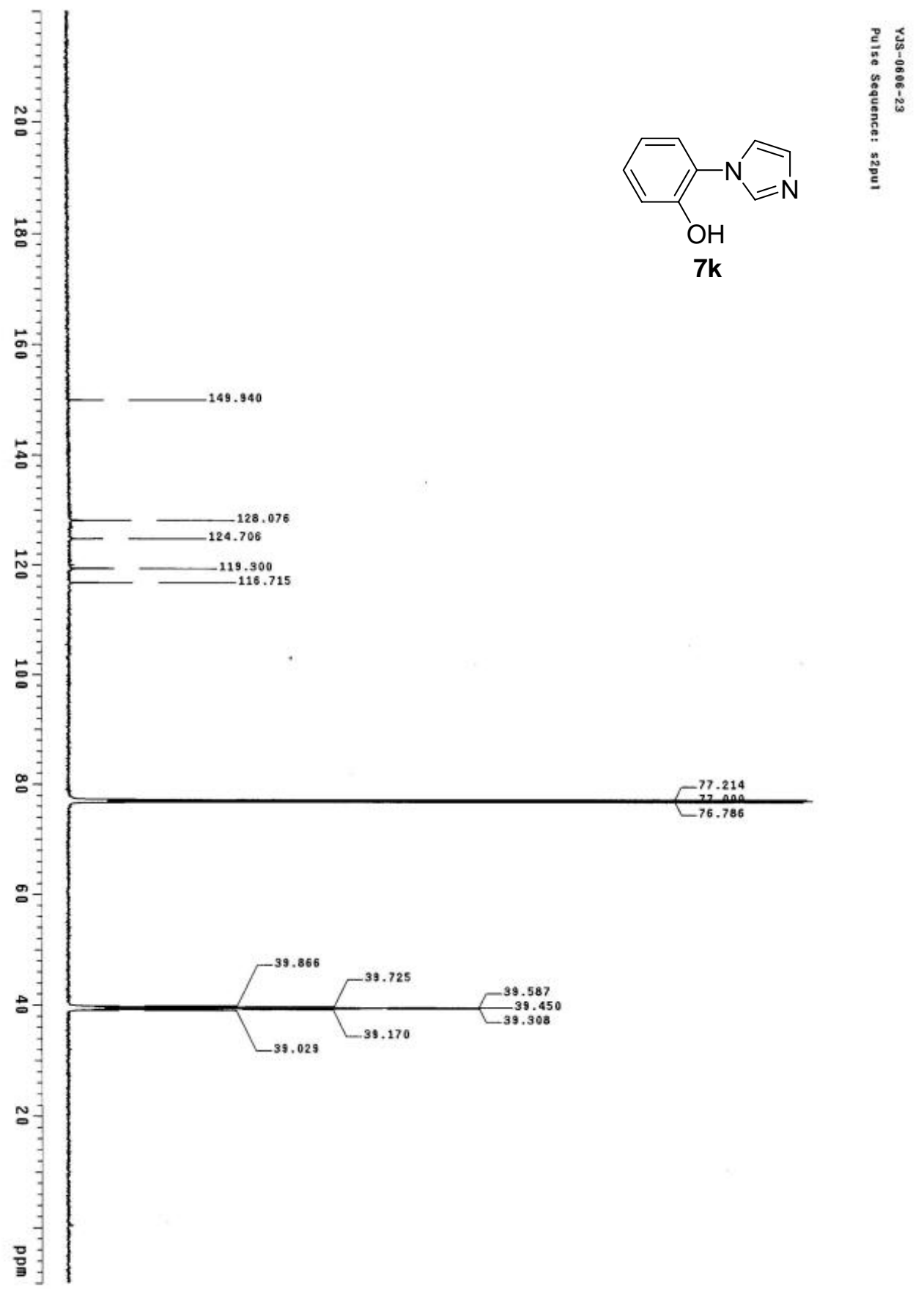




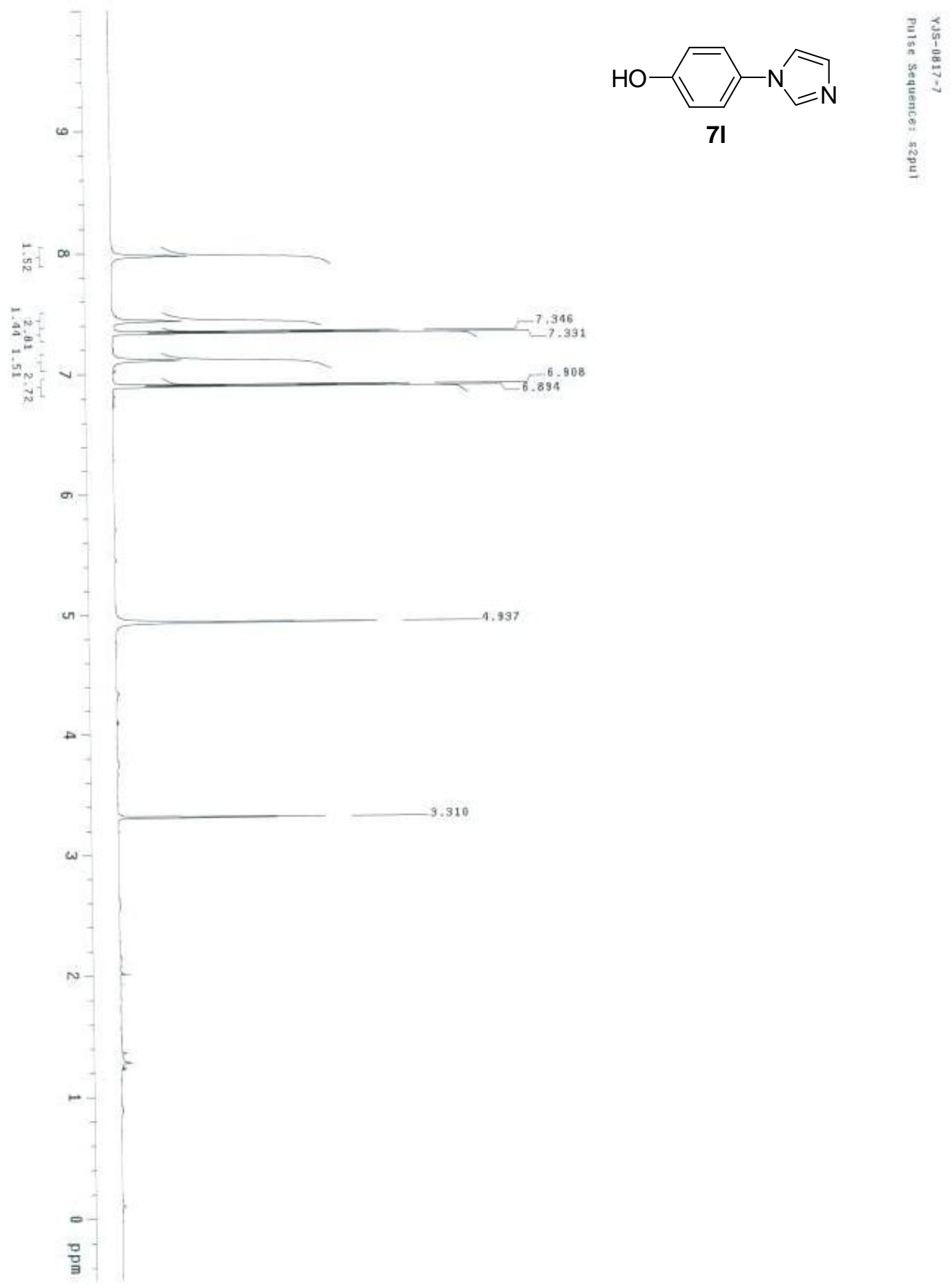




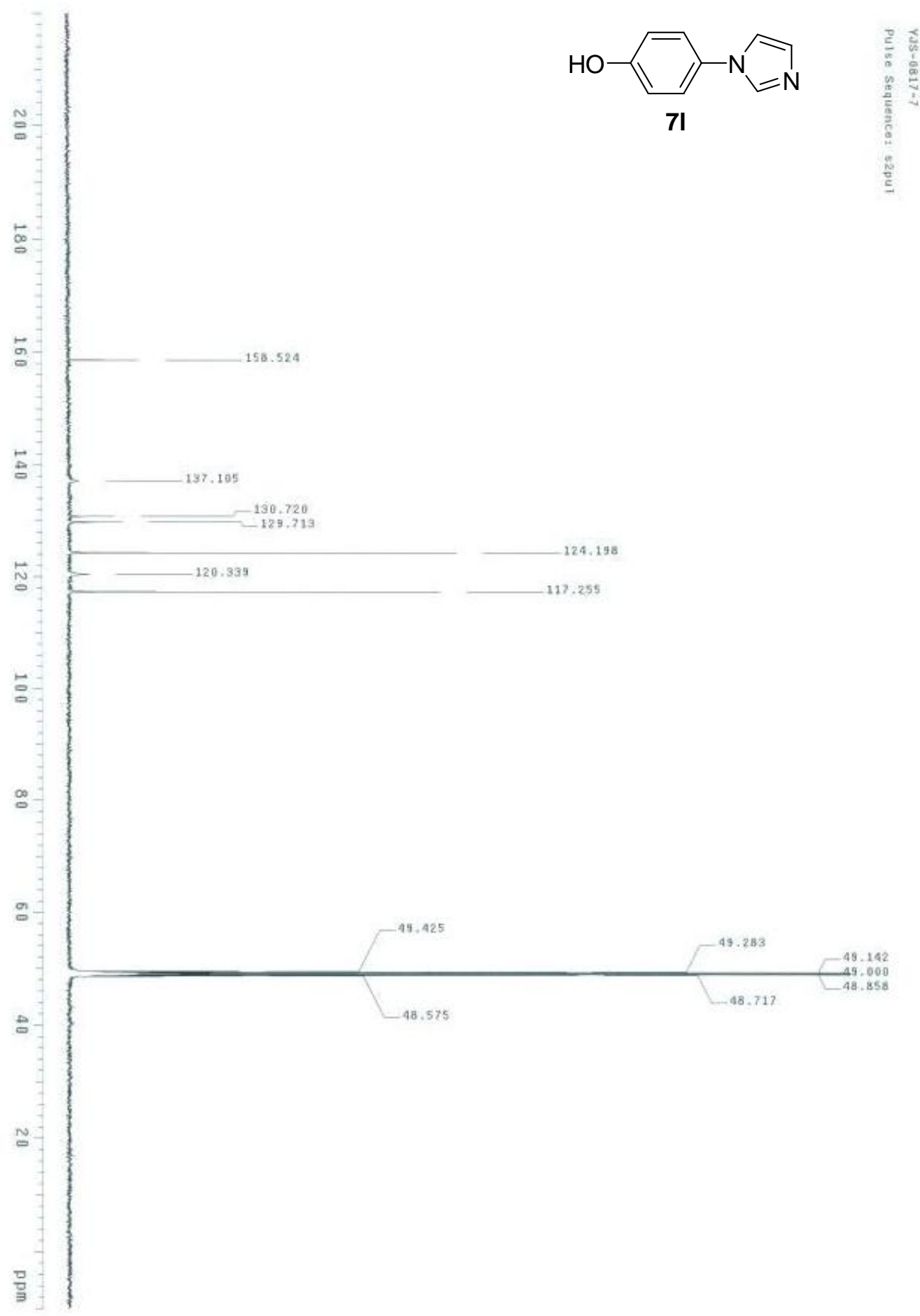




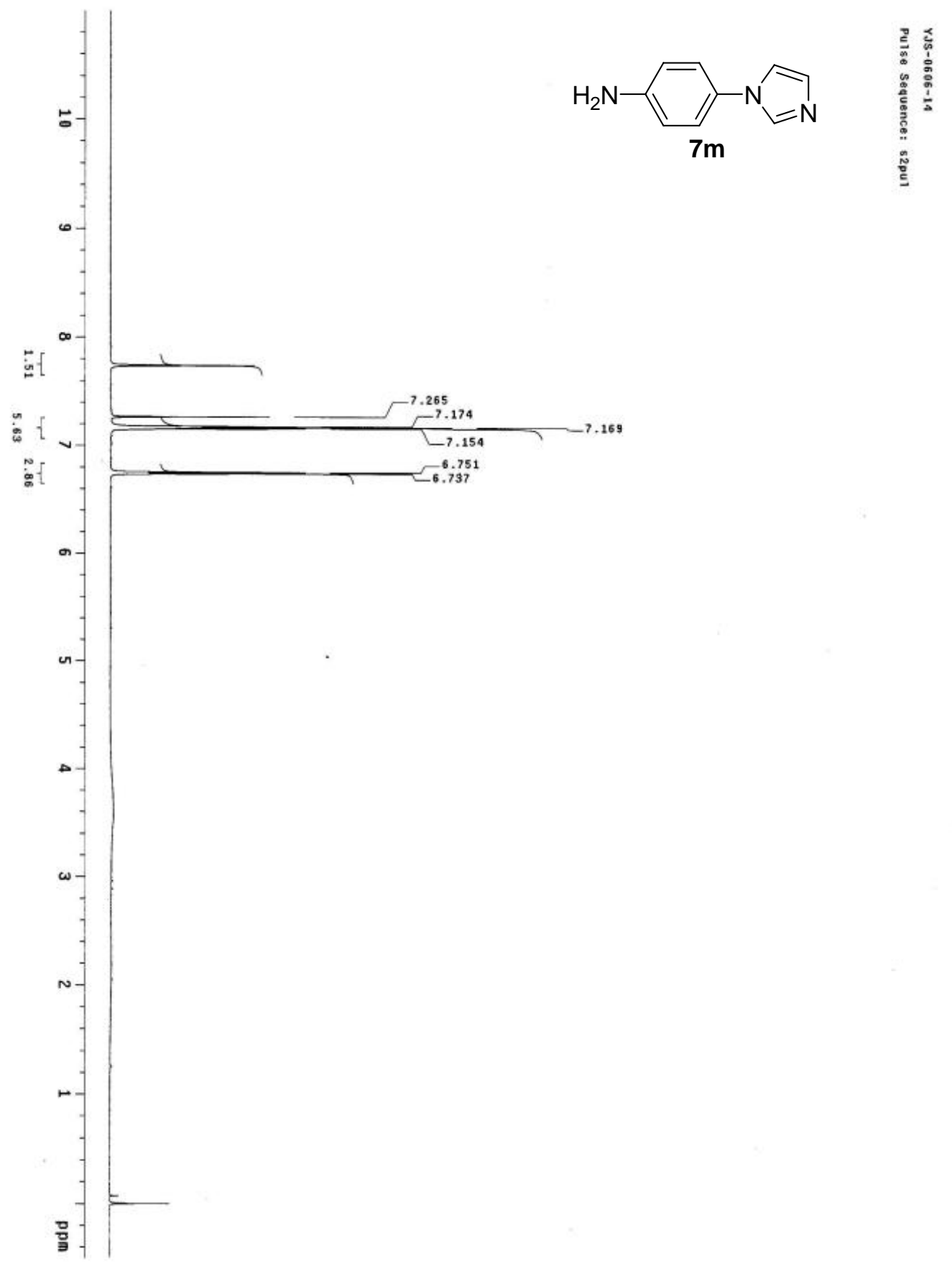



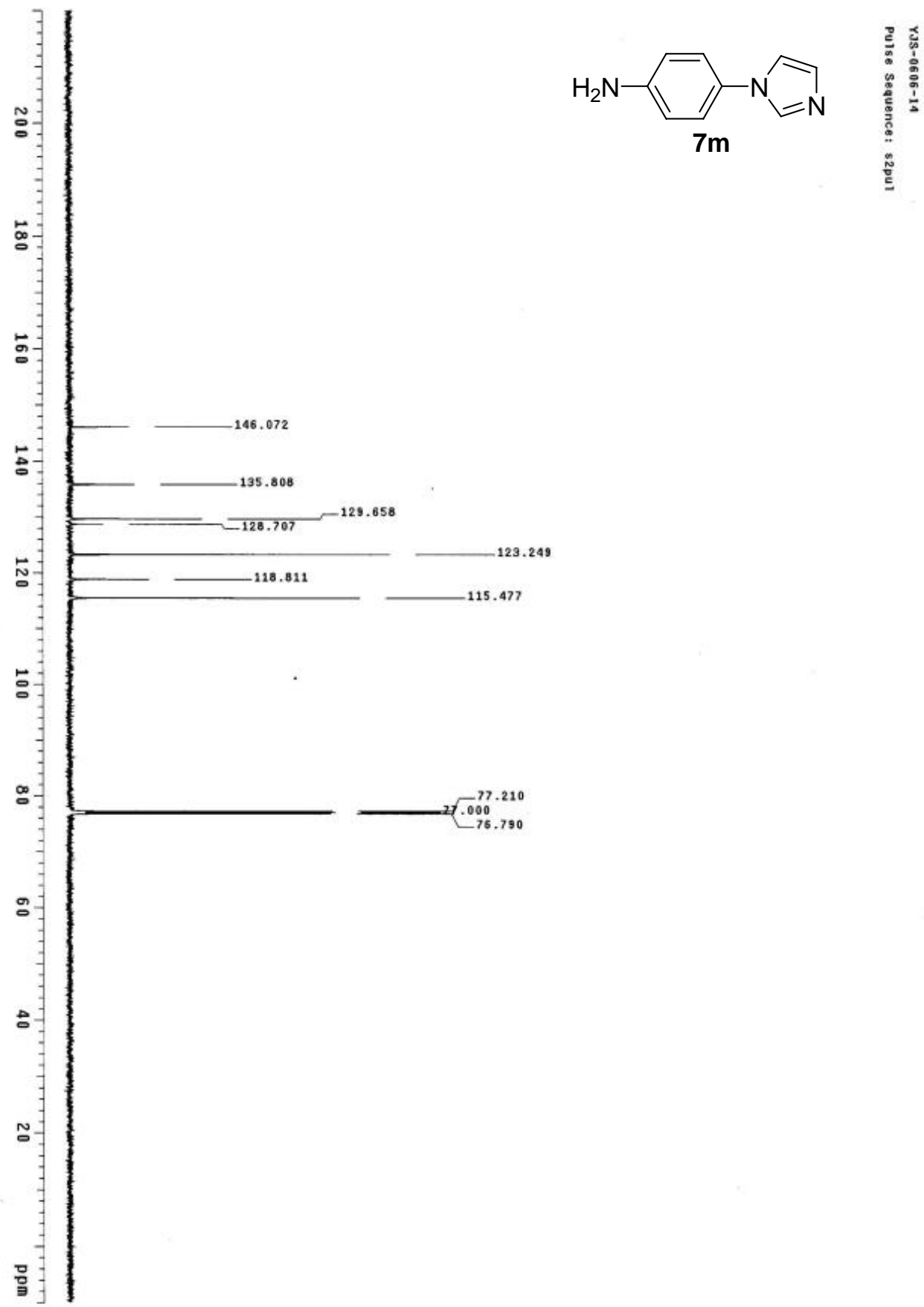

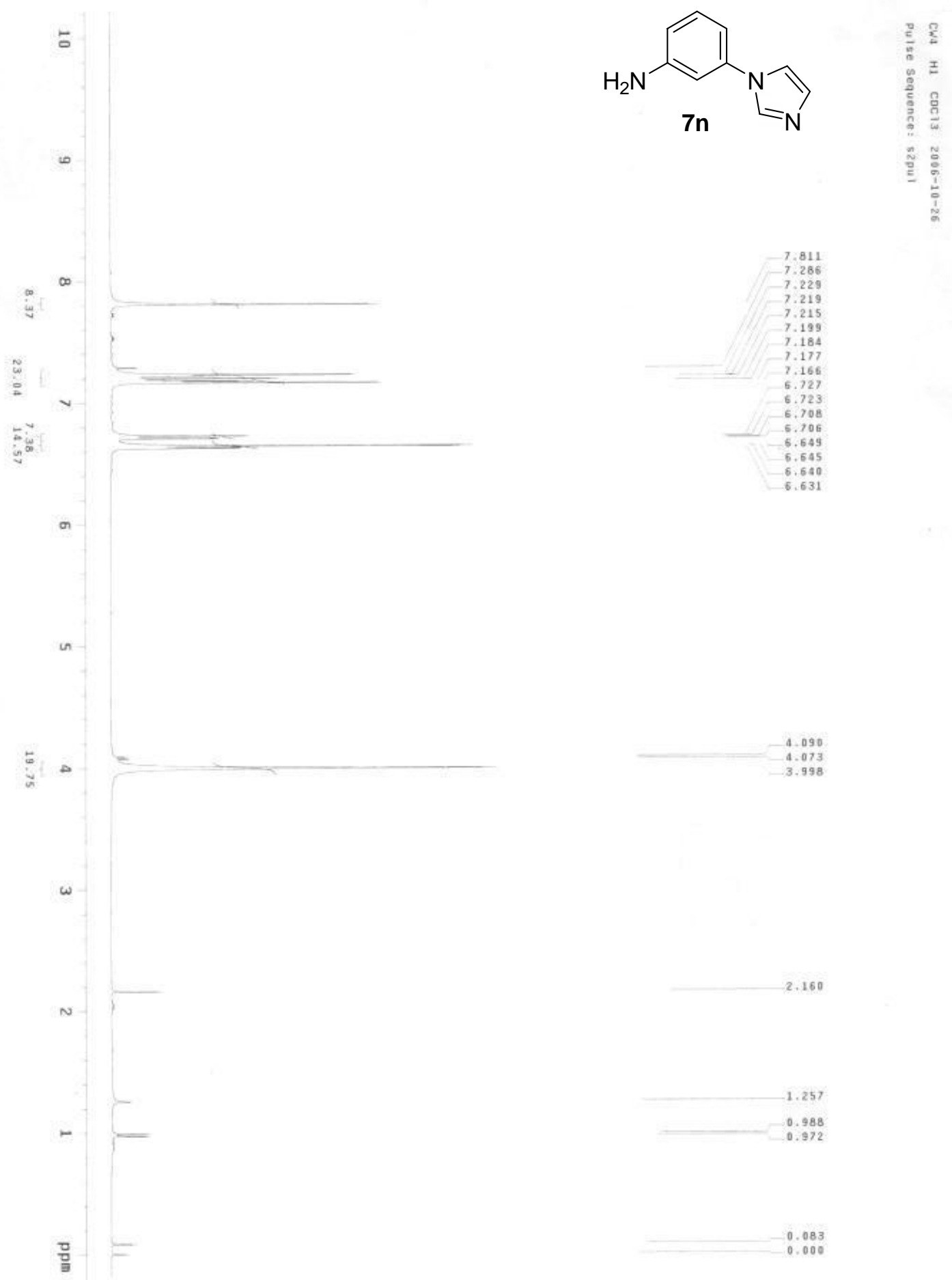

2.160

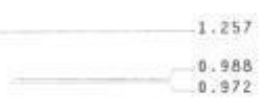




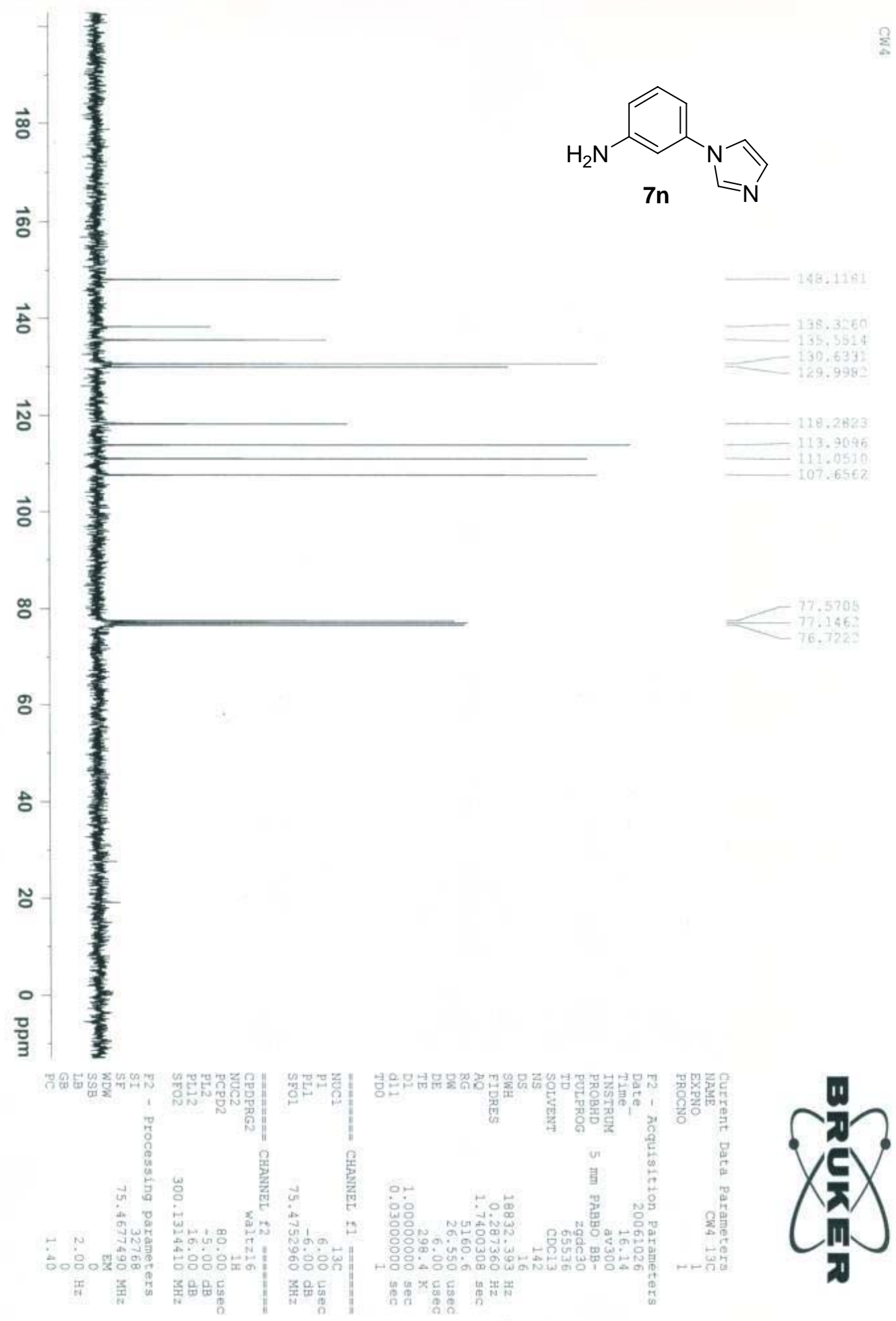




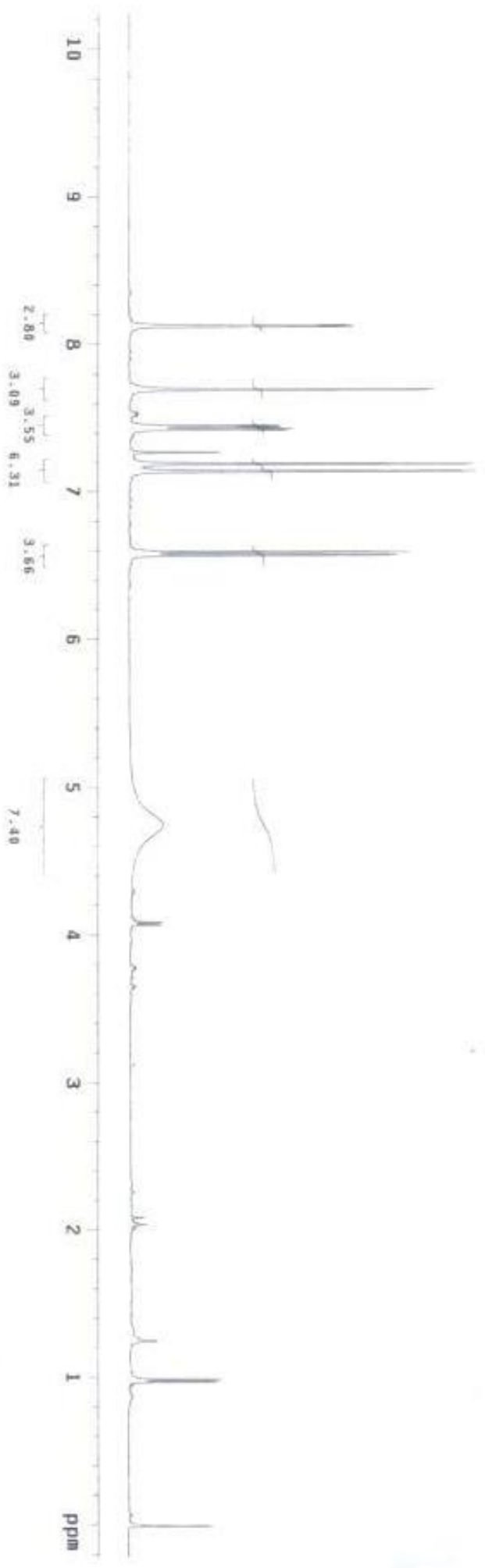

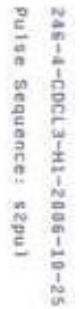
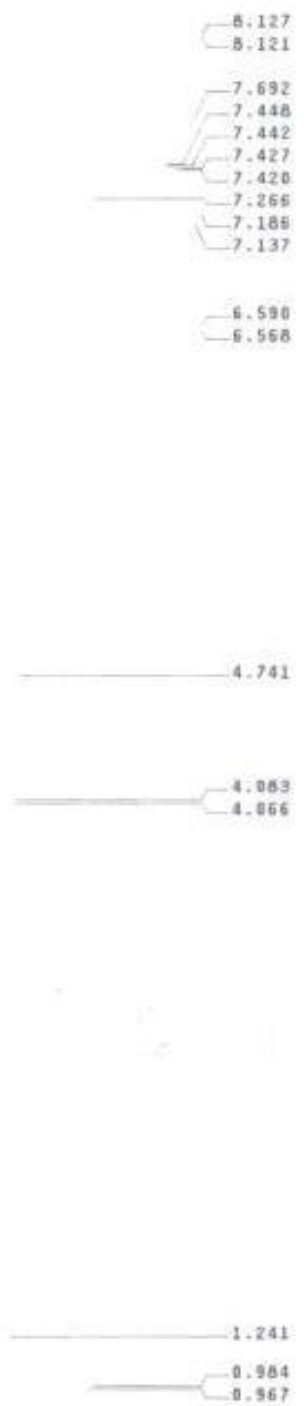

$-0.013$ 

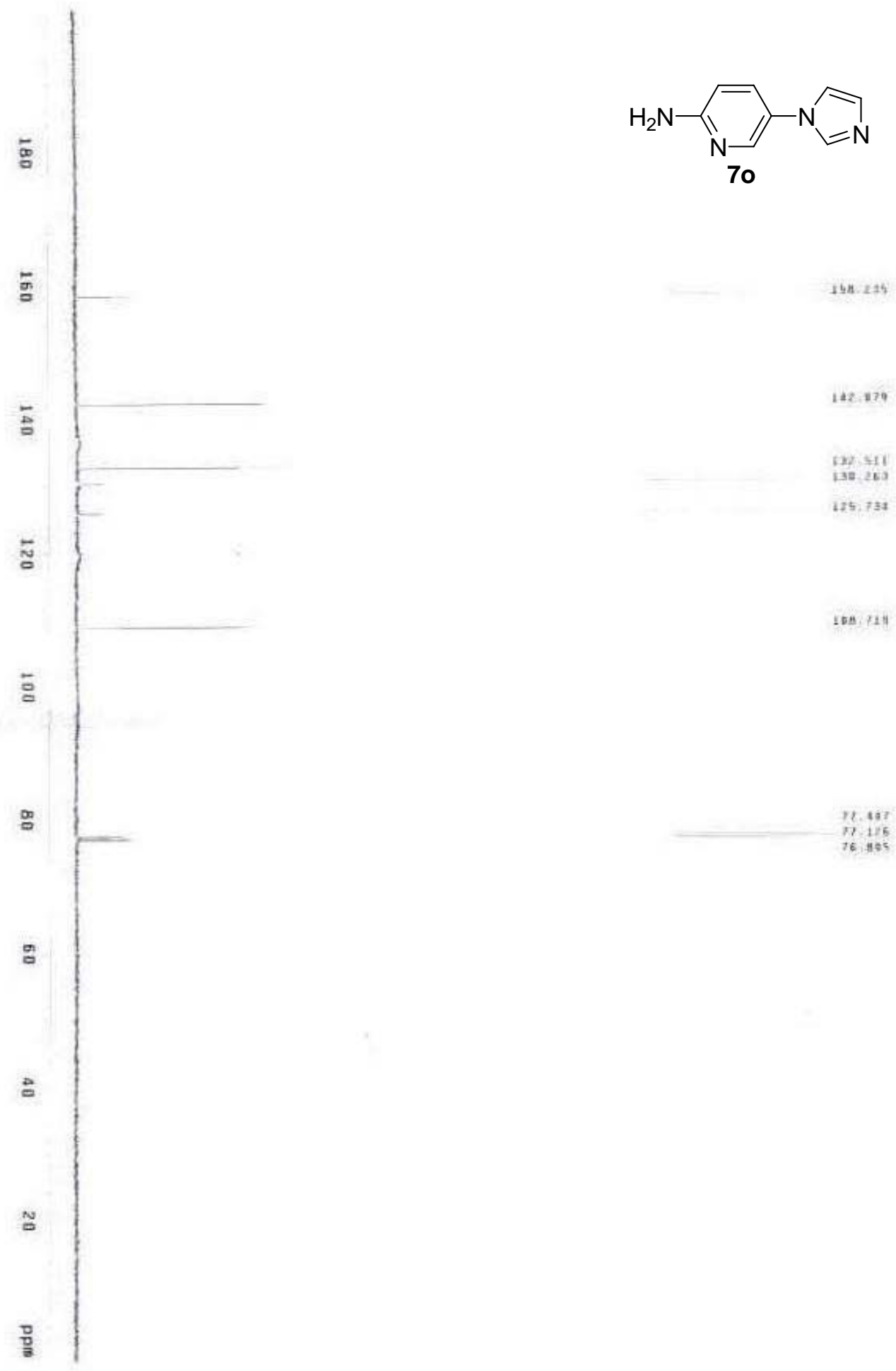


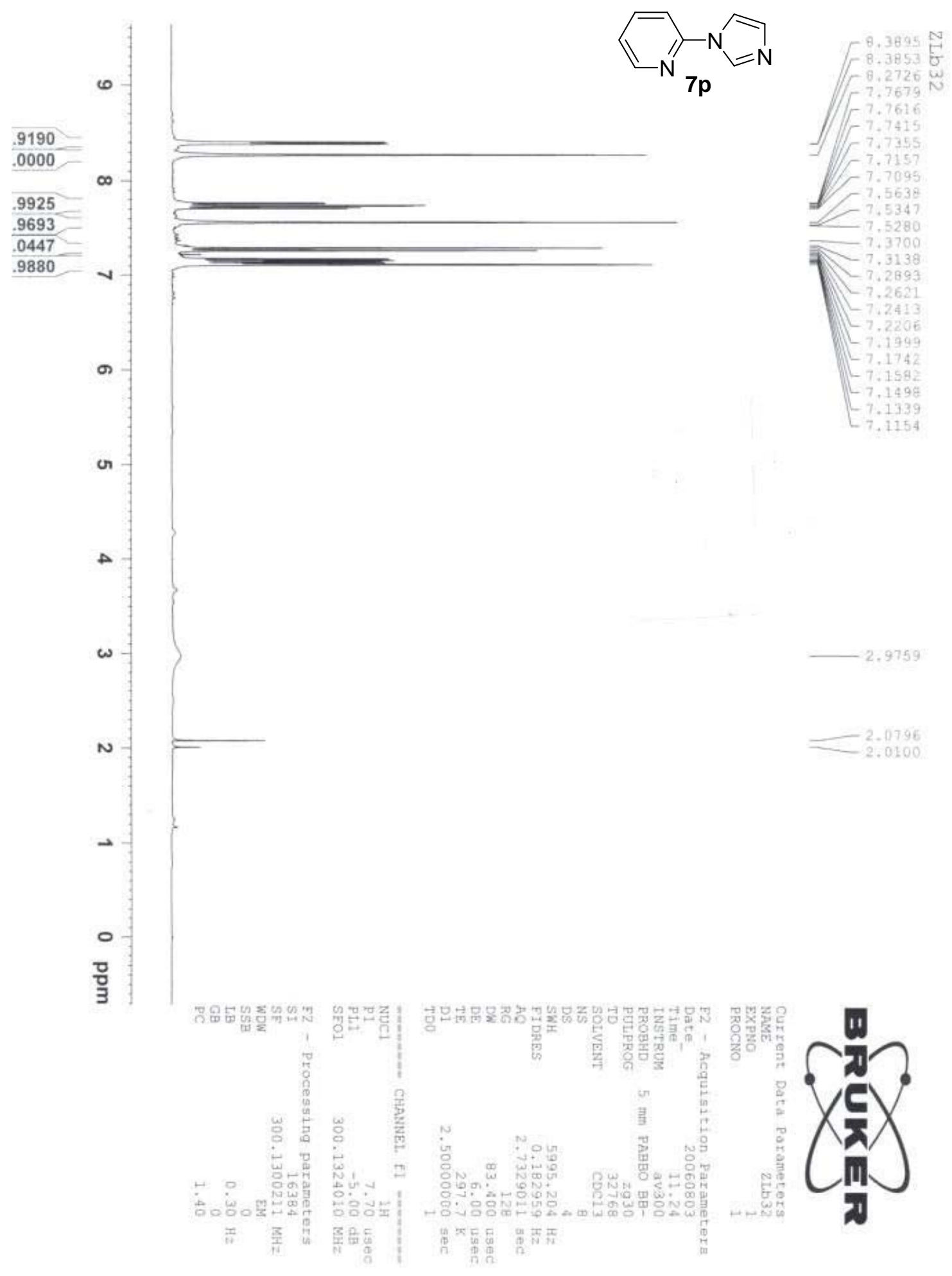




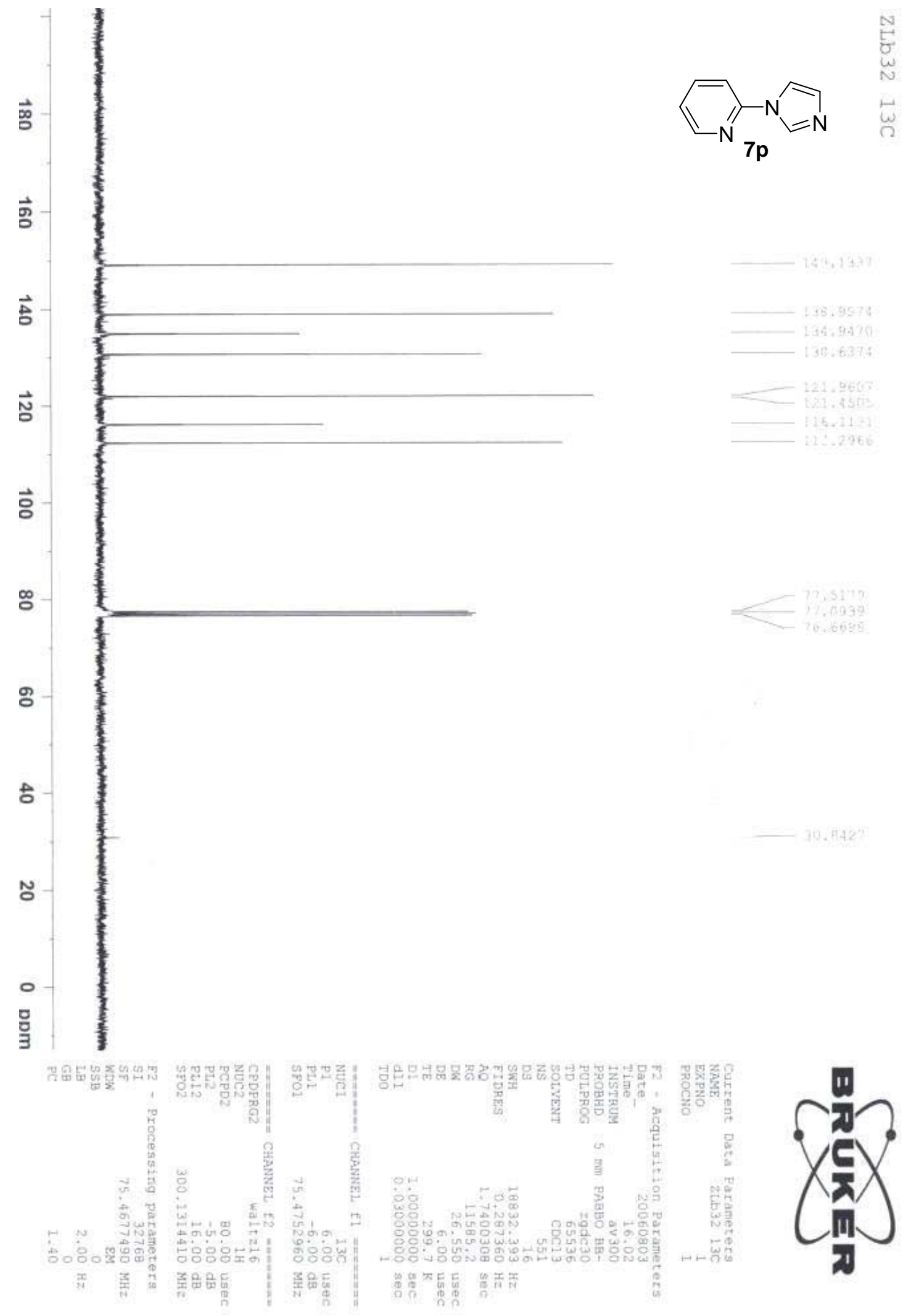



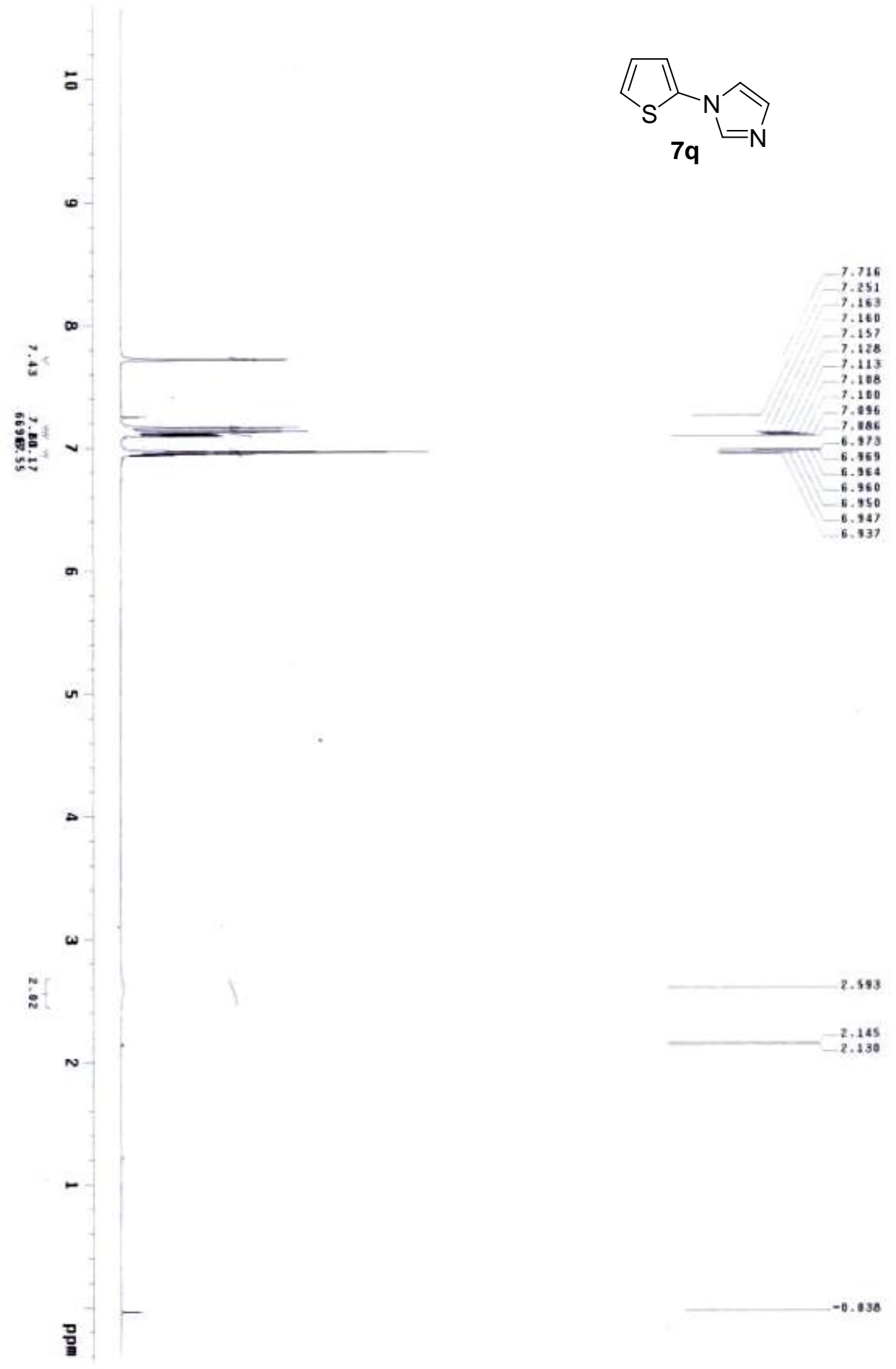


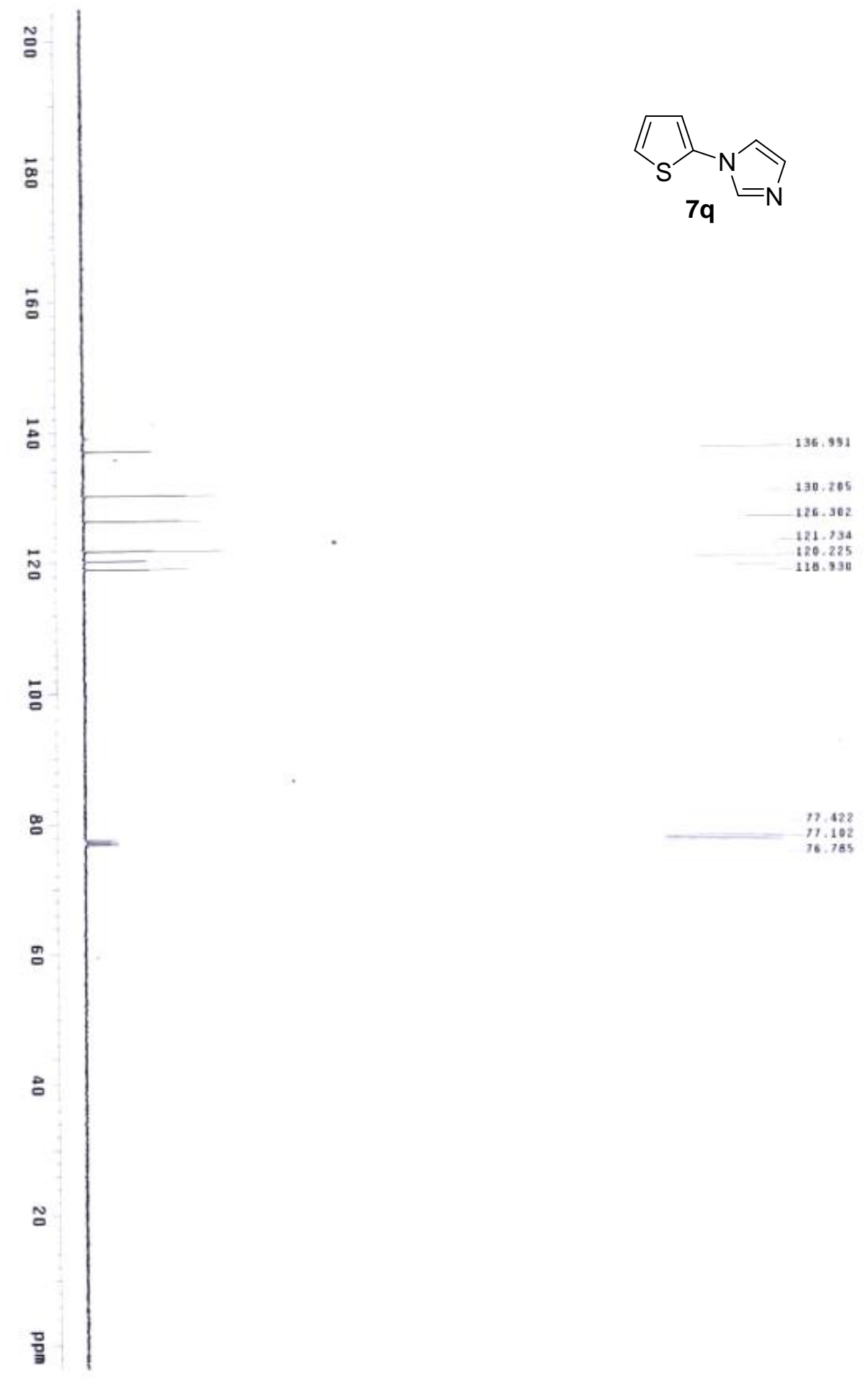

0
0
0 

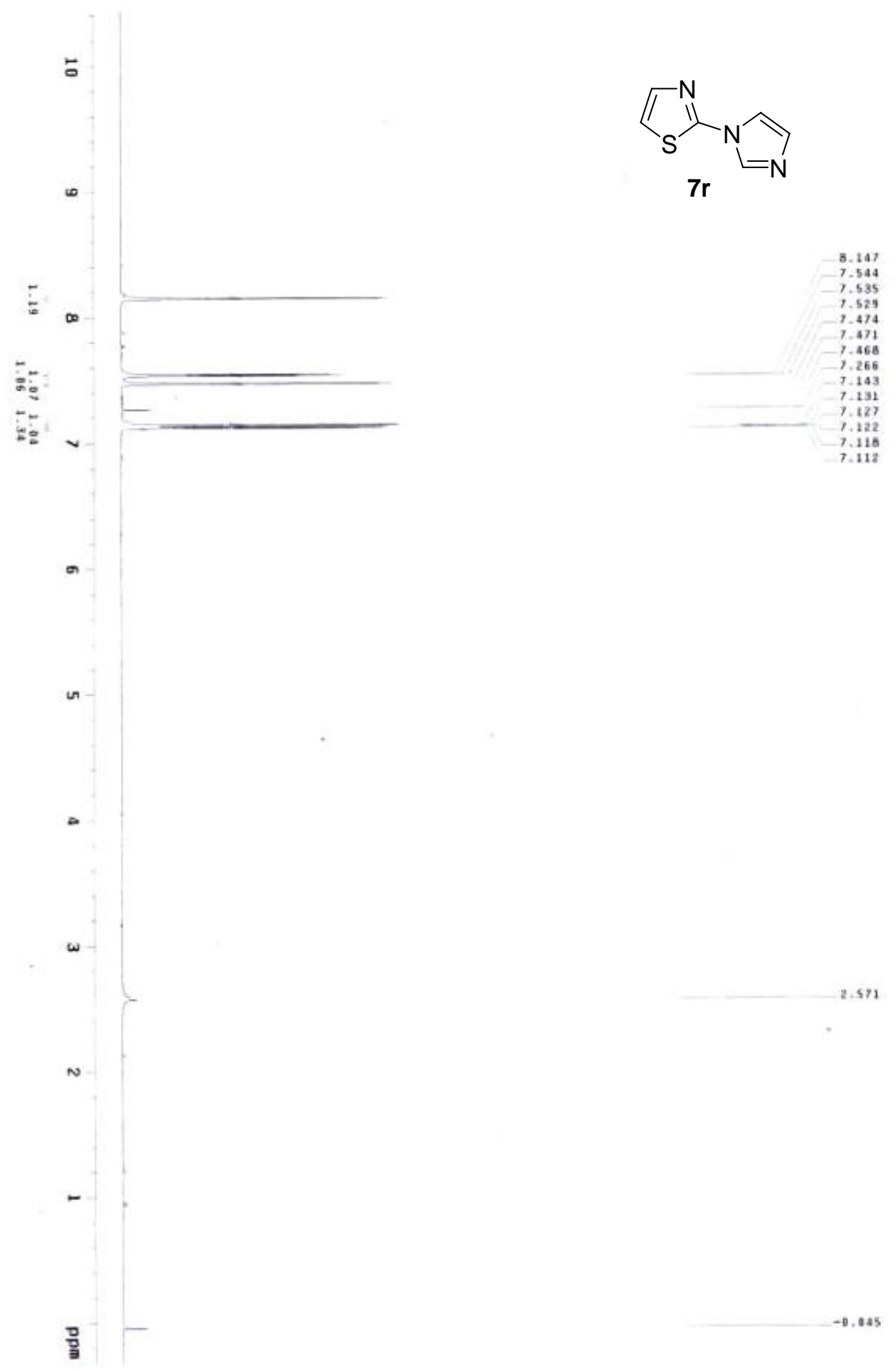

2.571 

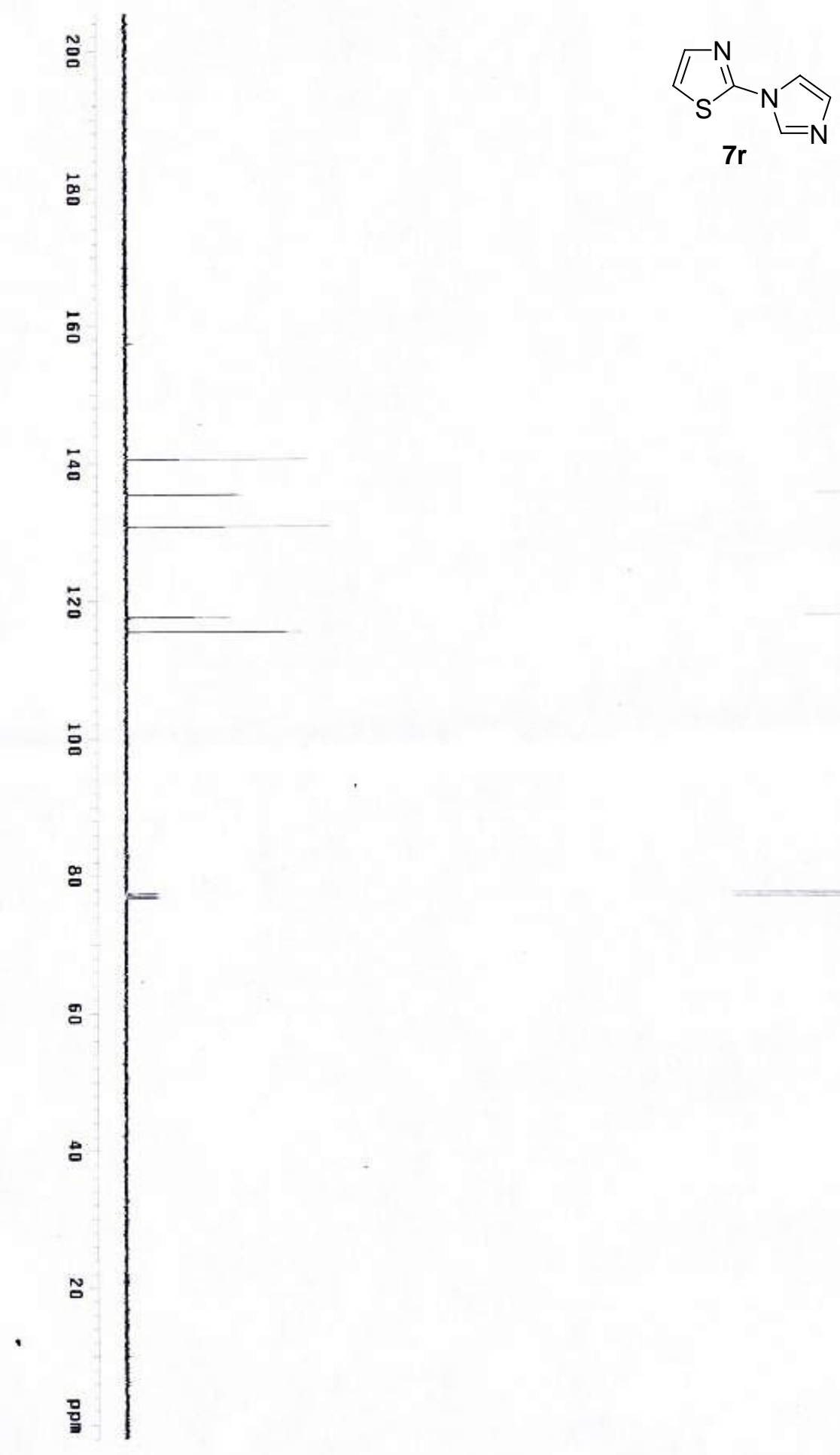

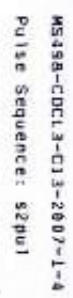

140.523

135.436

130.776

117.625
115.508

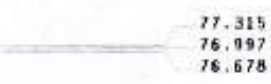




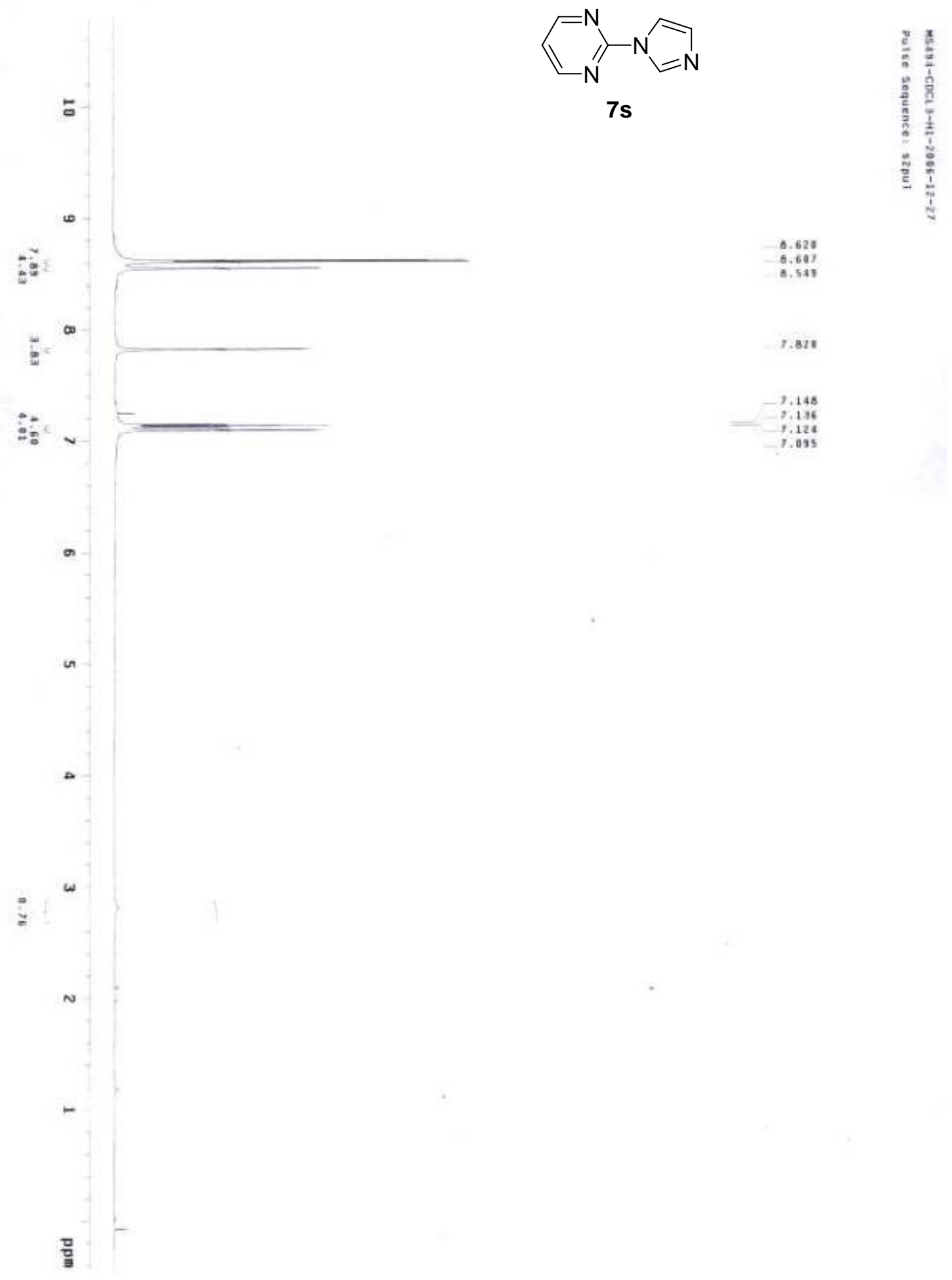



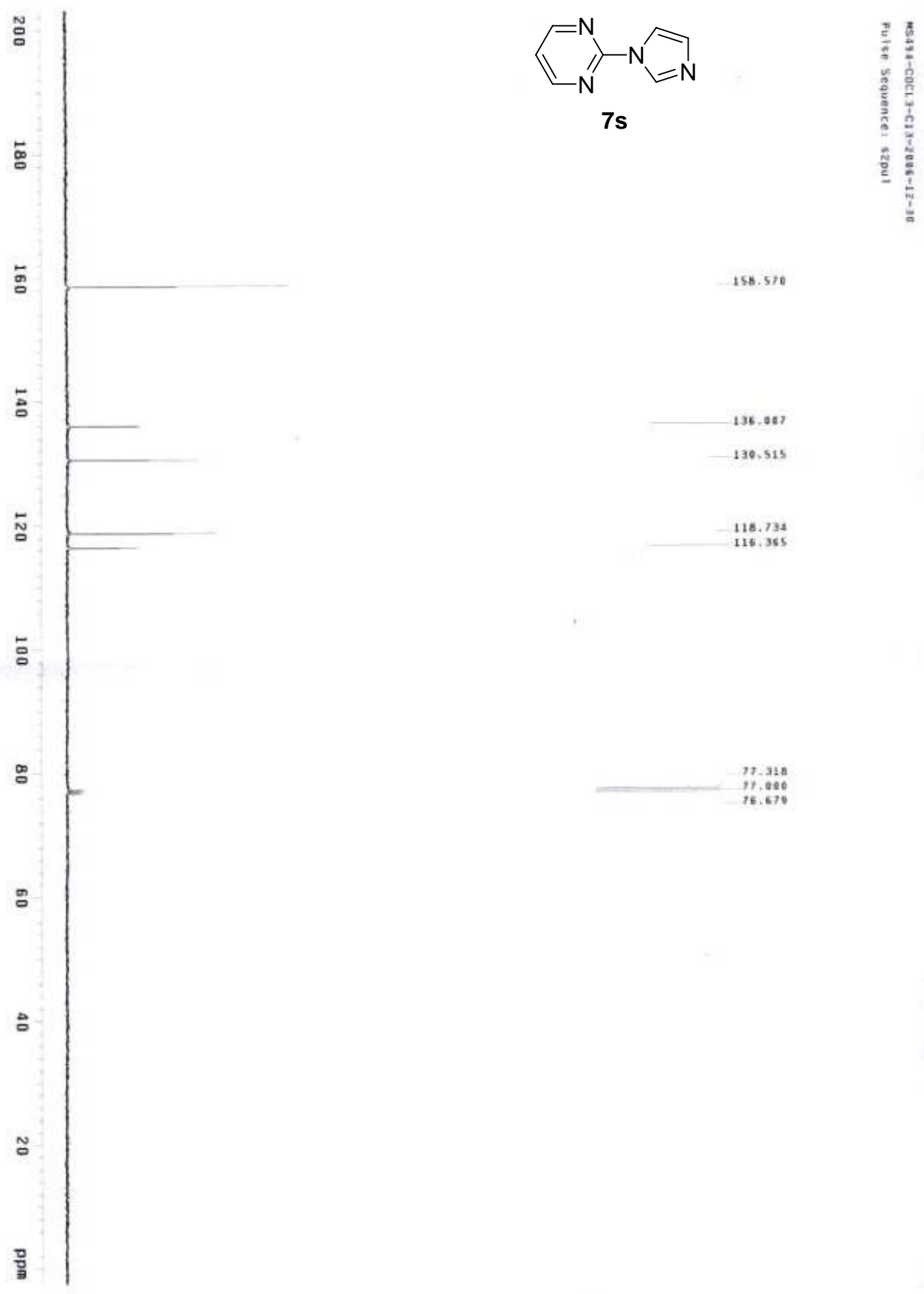

158.570

136.067

130.515

116,355

77. 318

i), ese

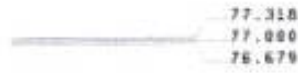




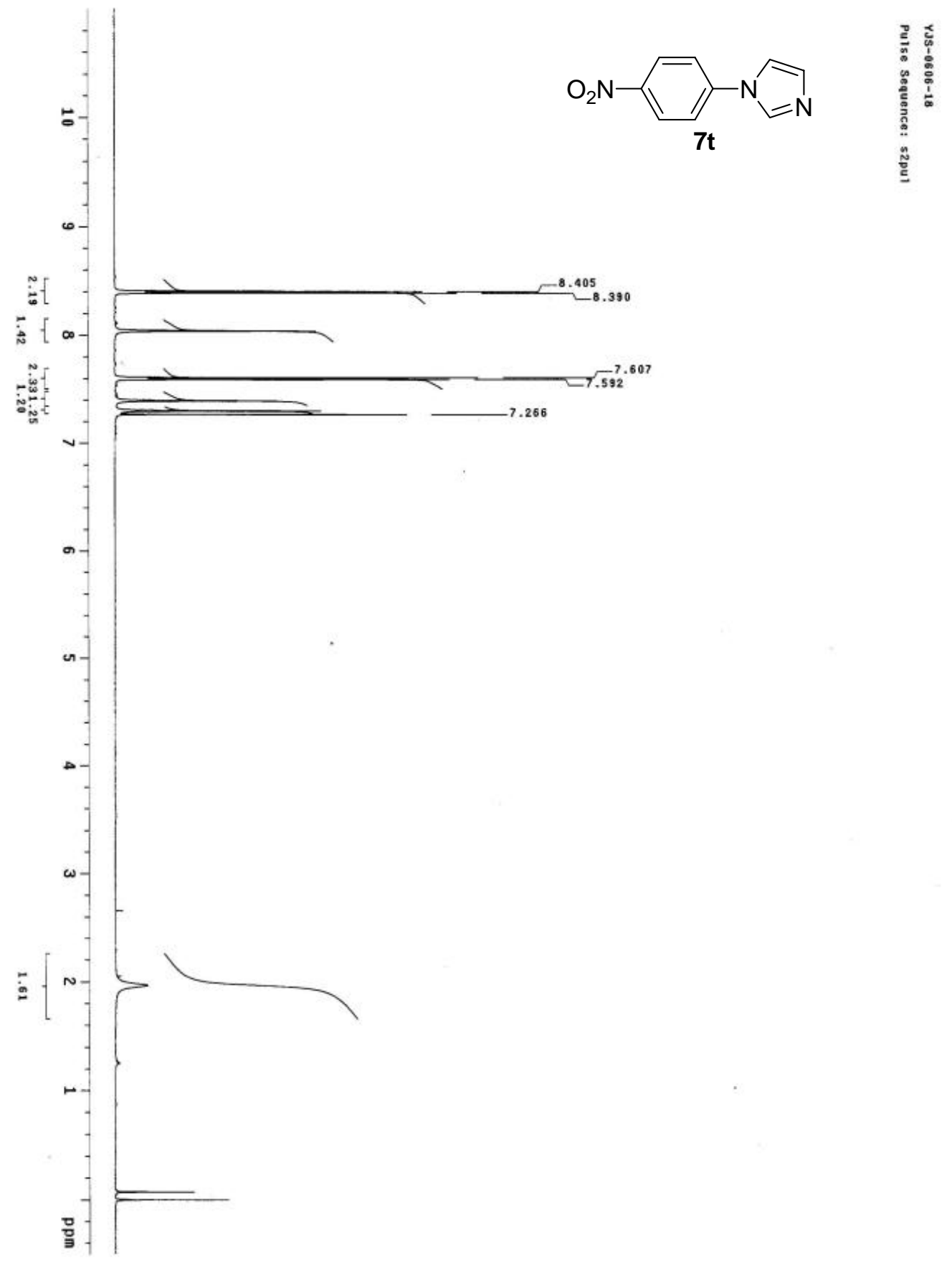




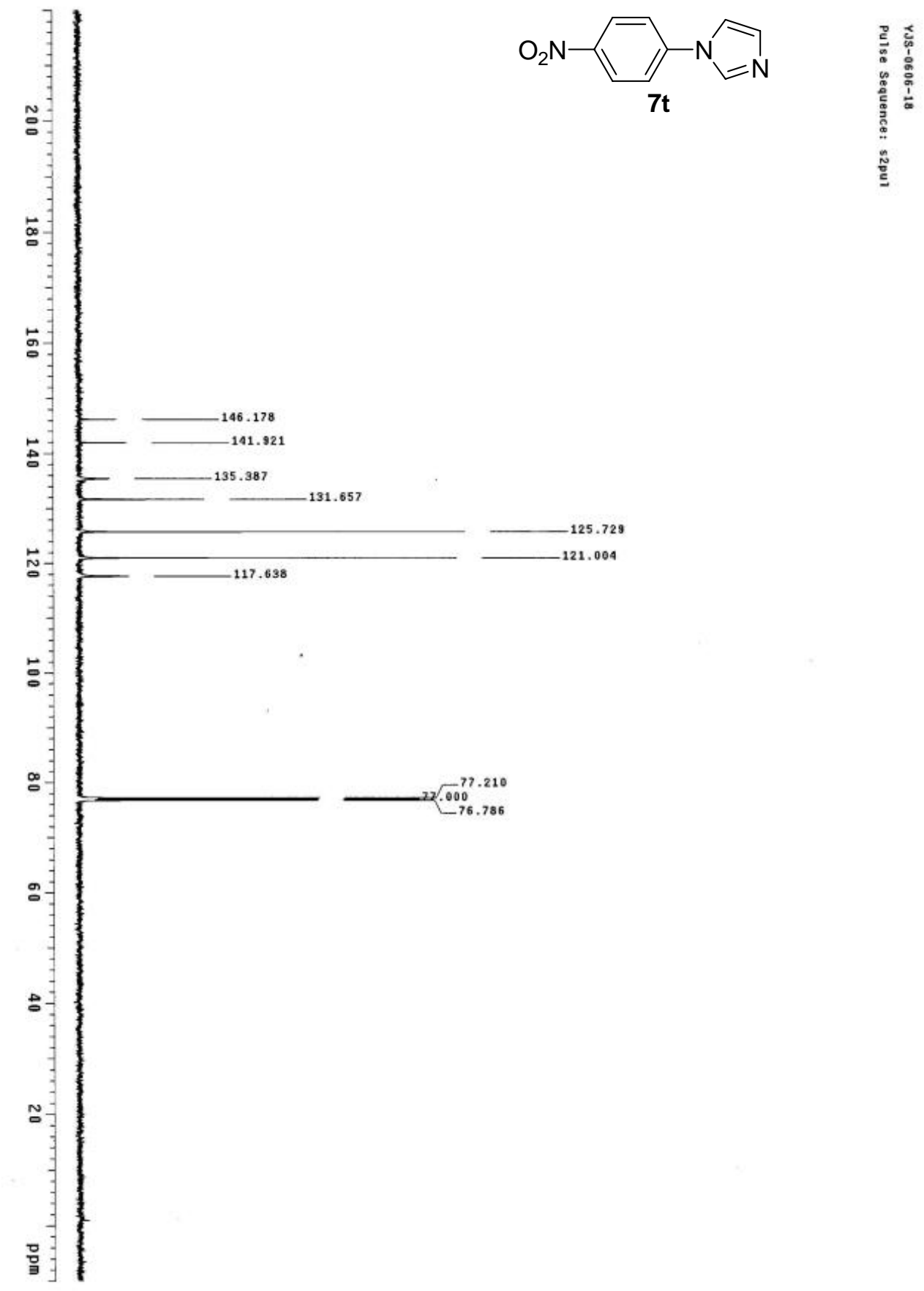




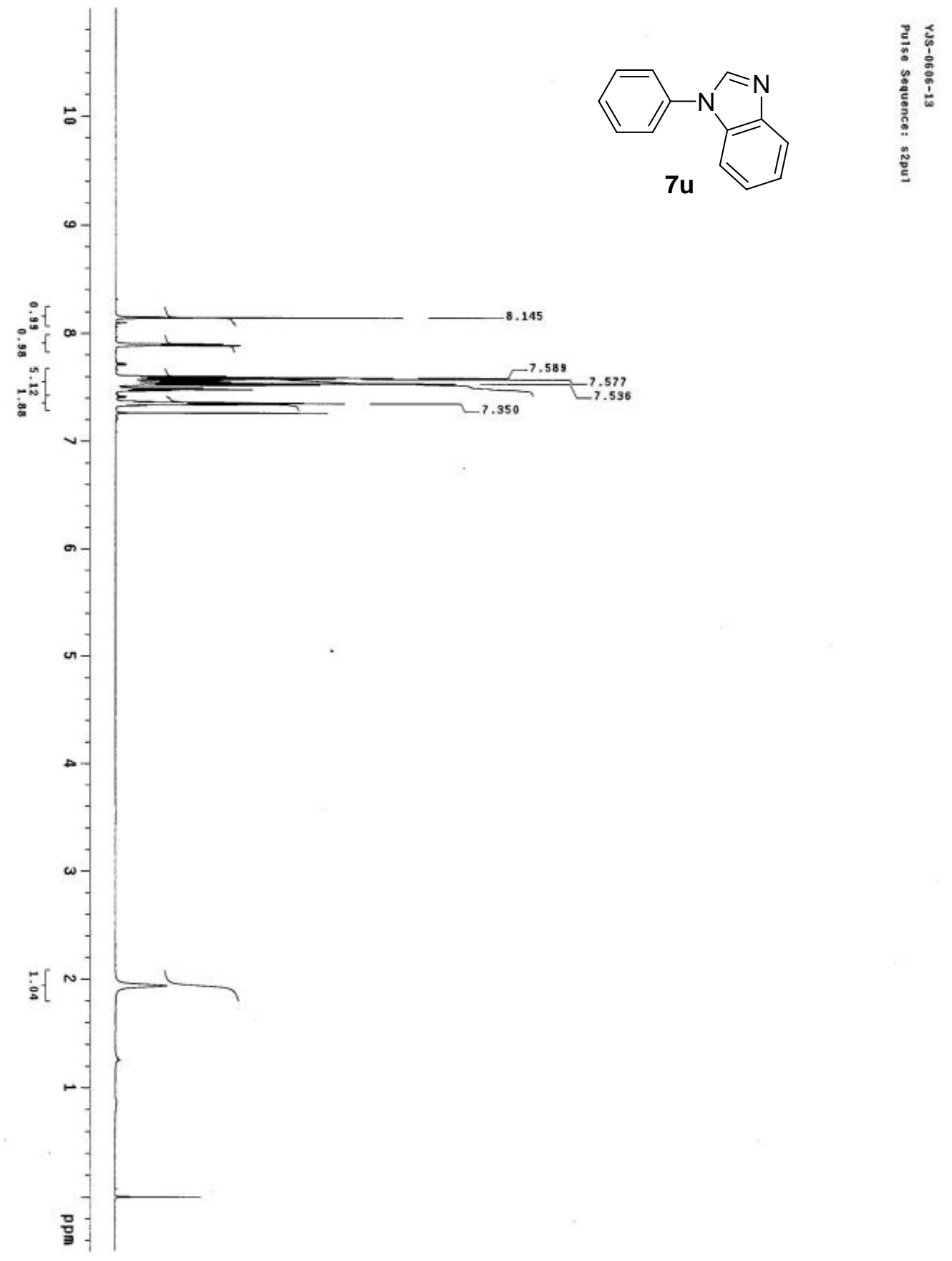




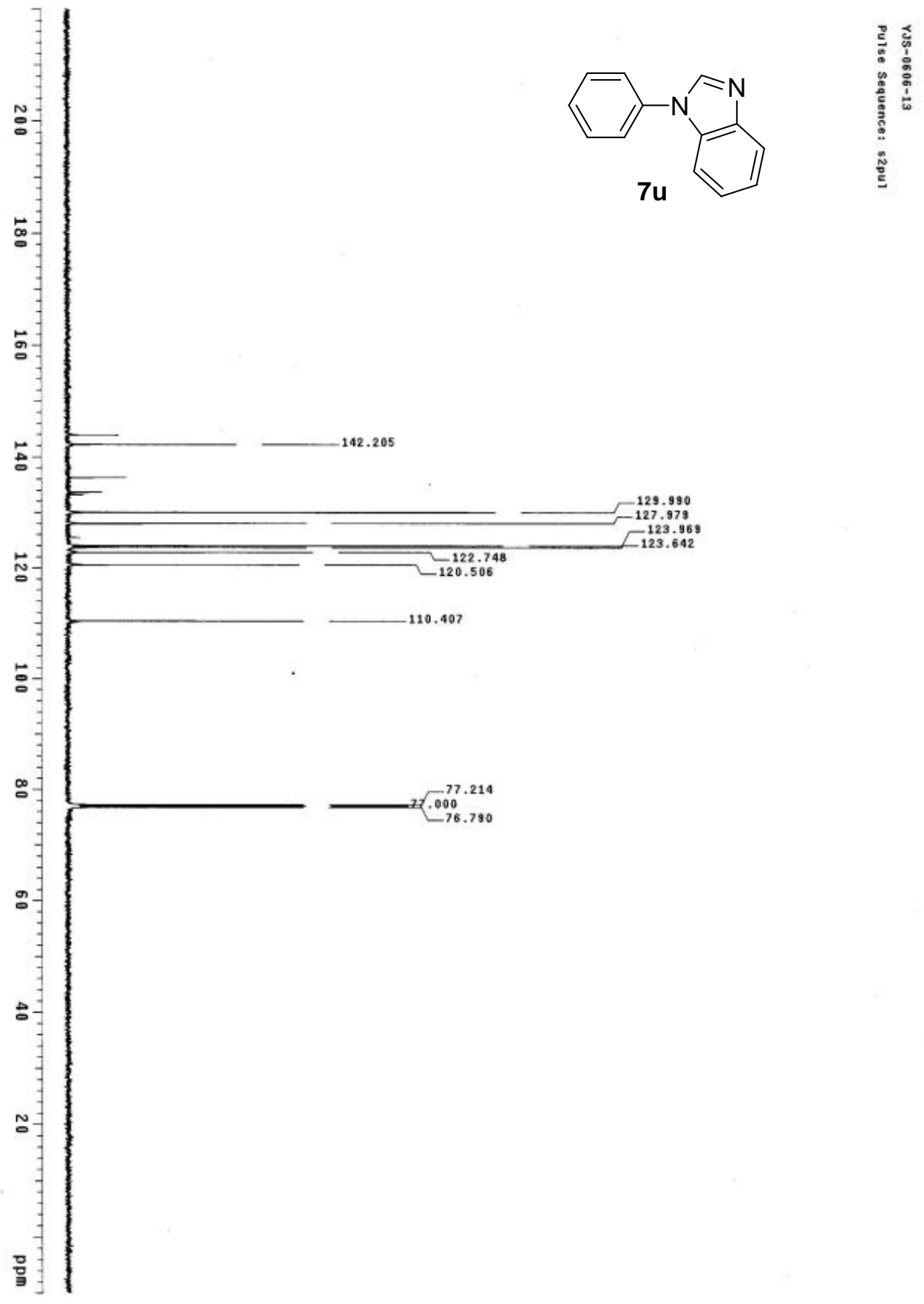




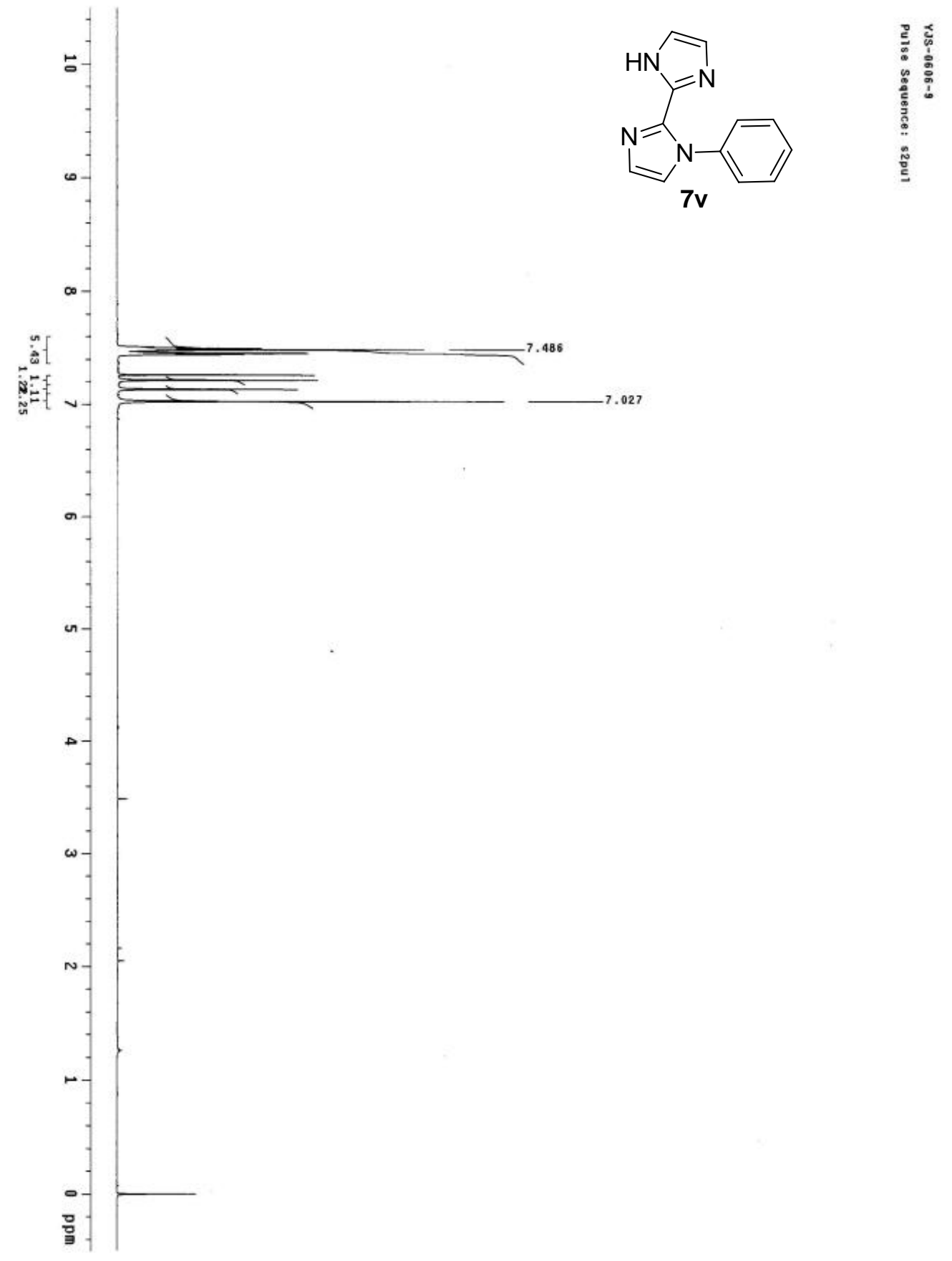




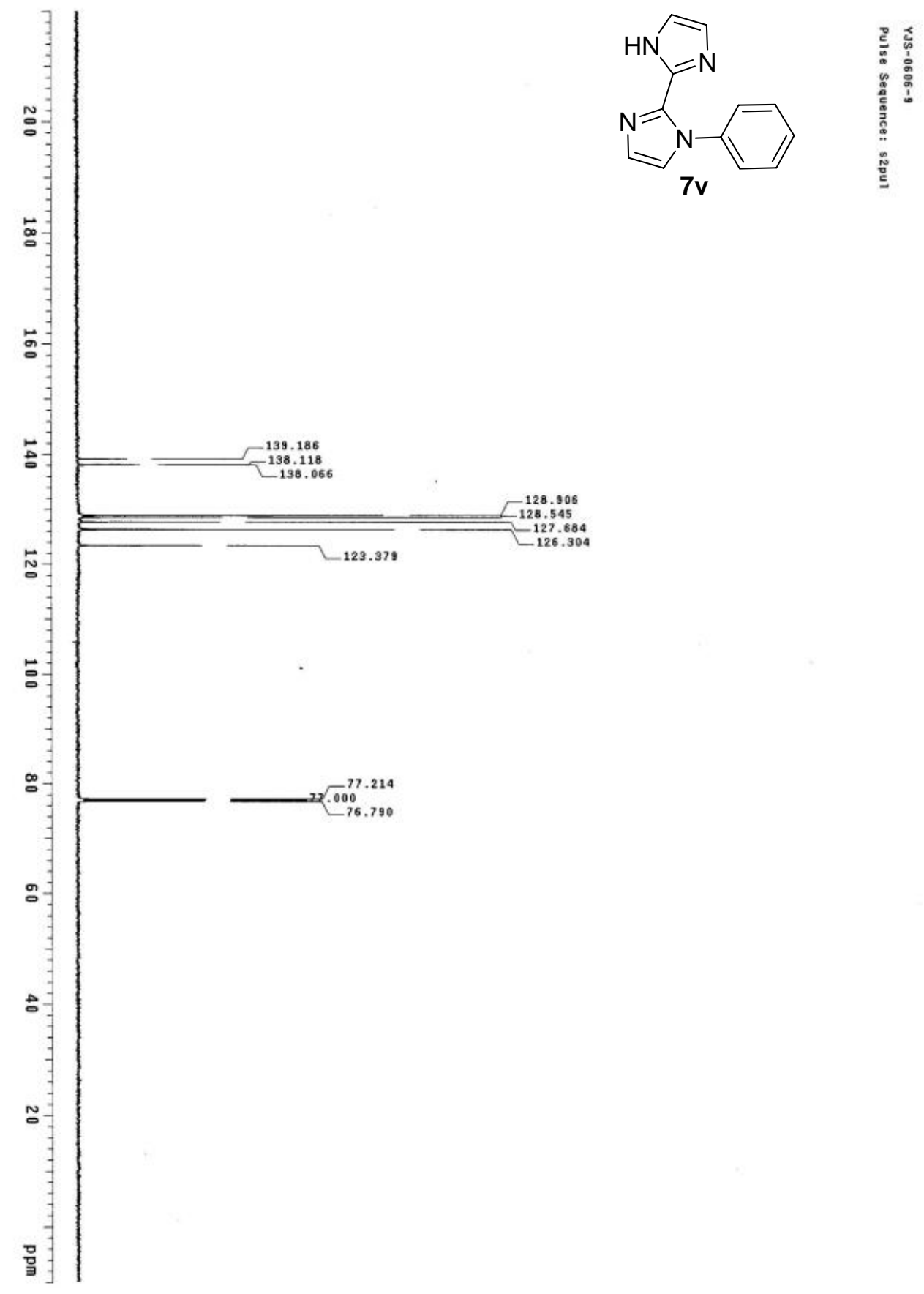




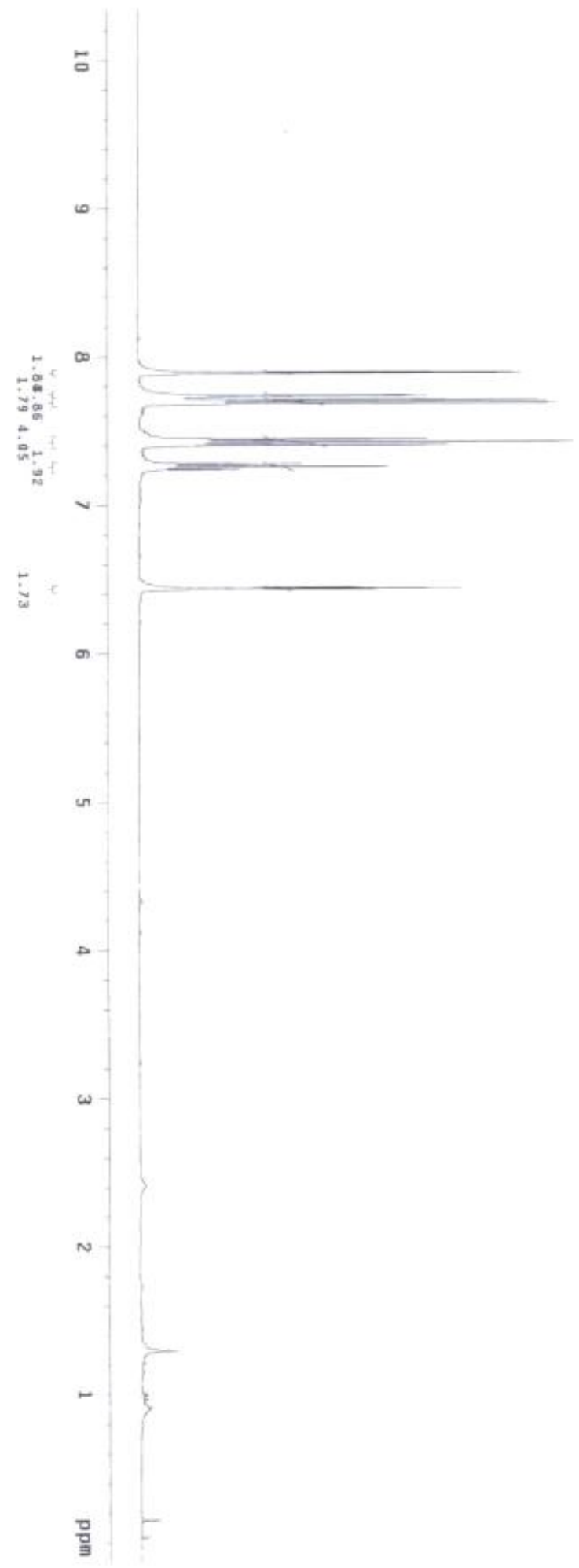




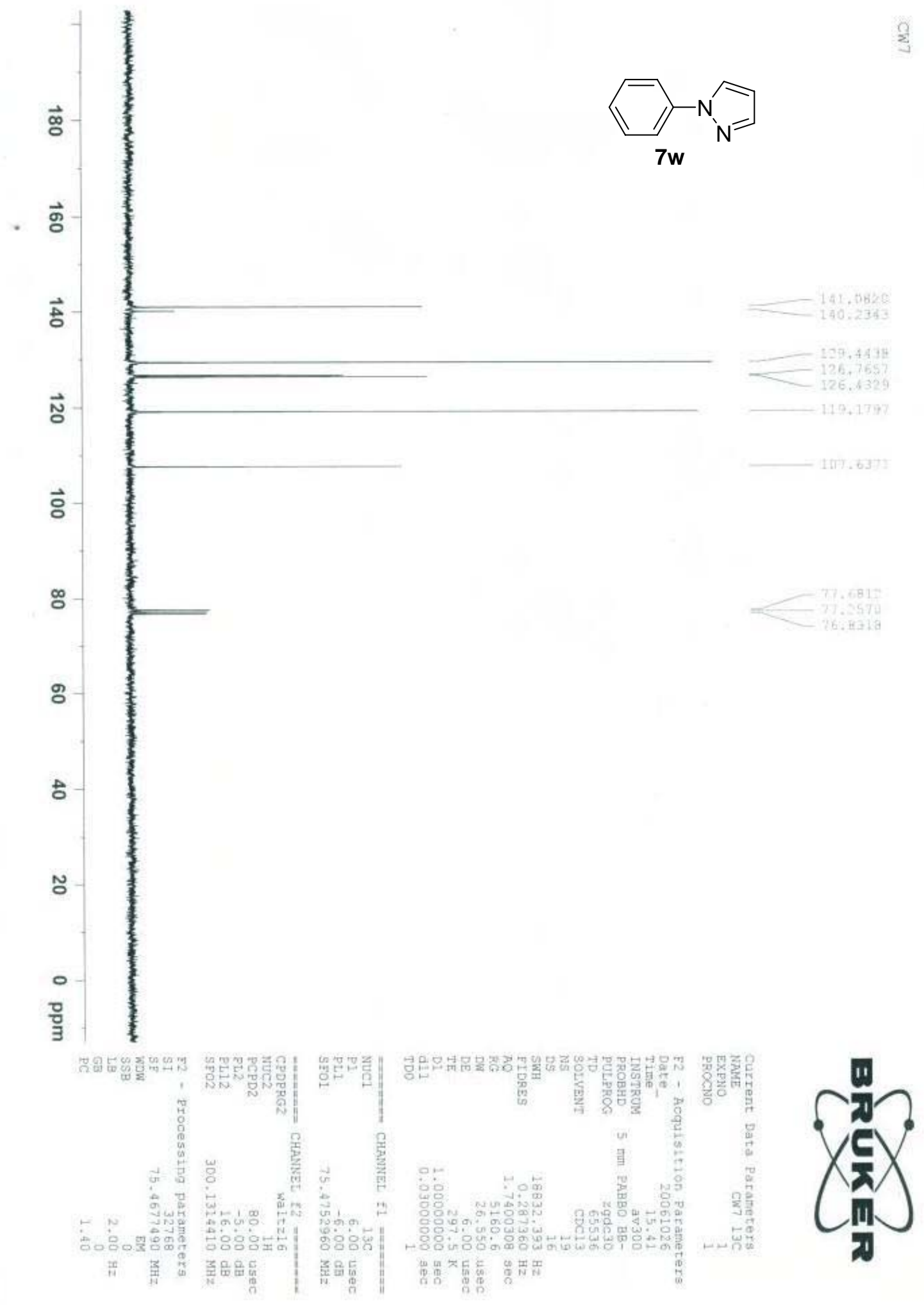




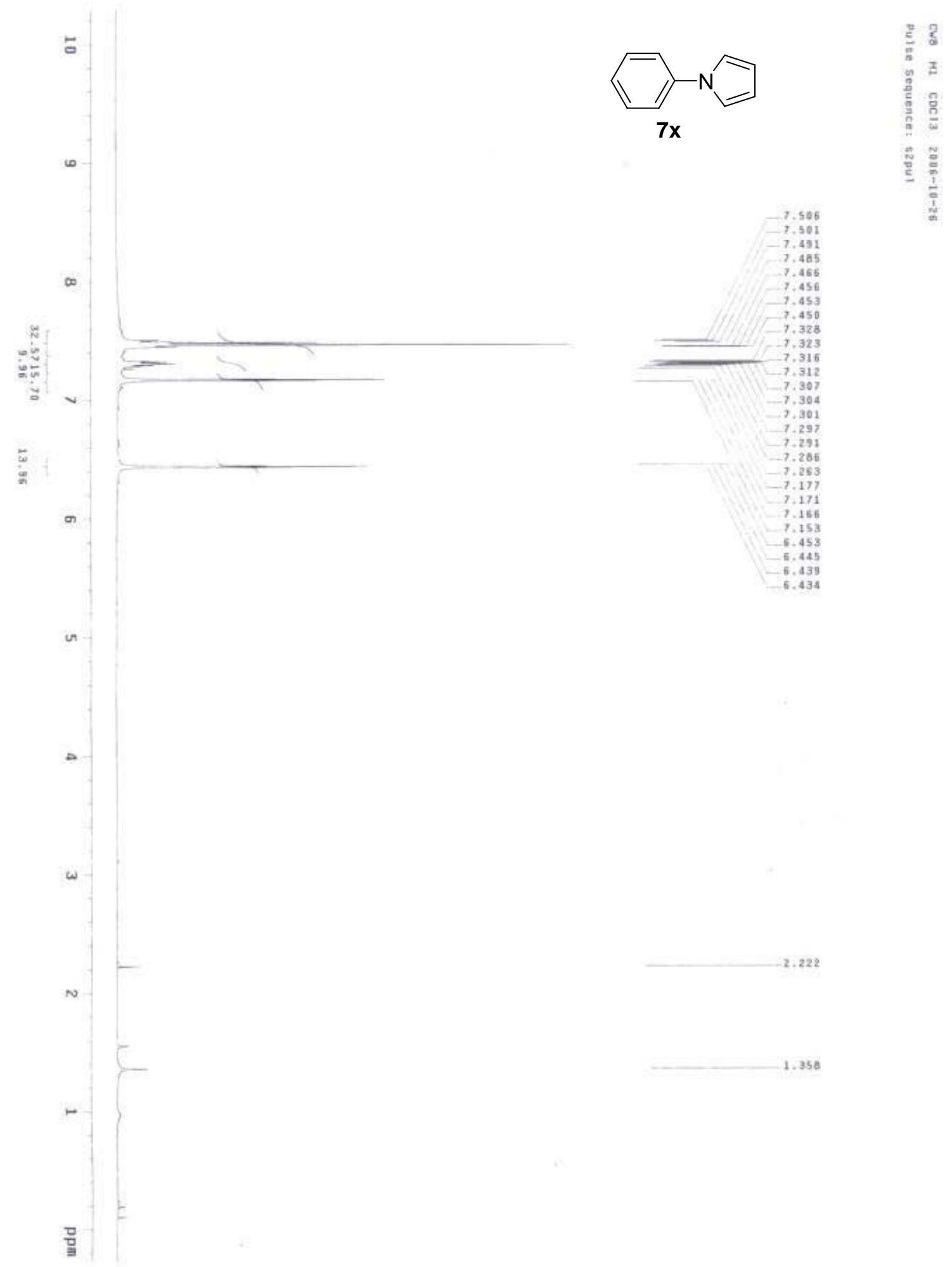




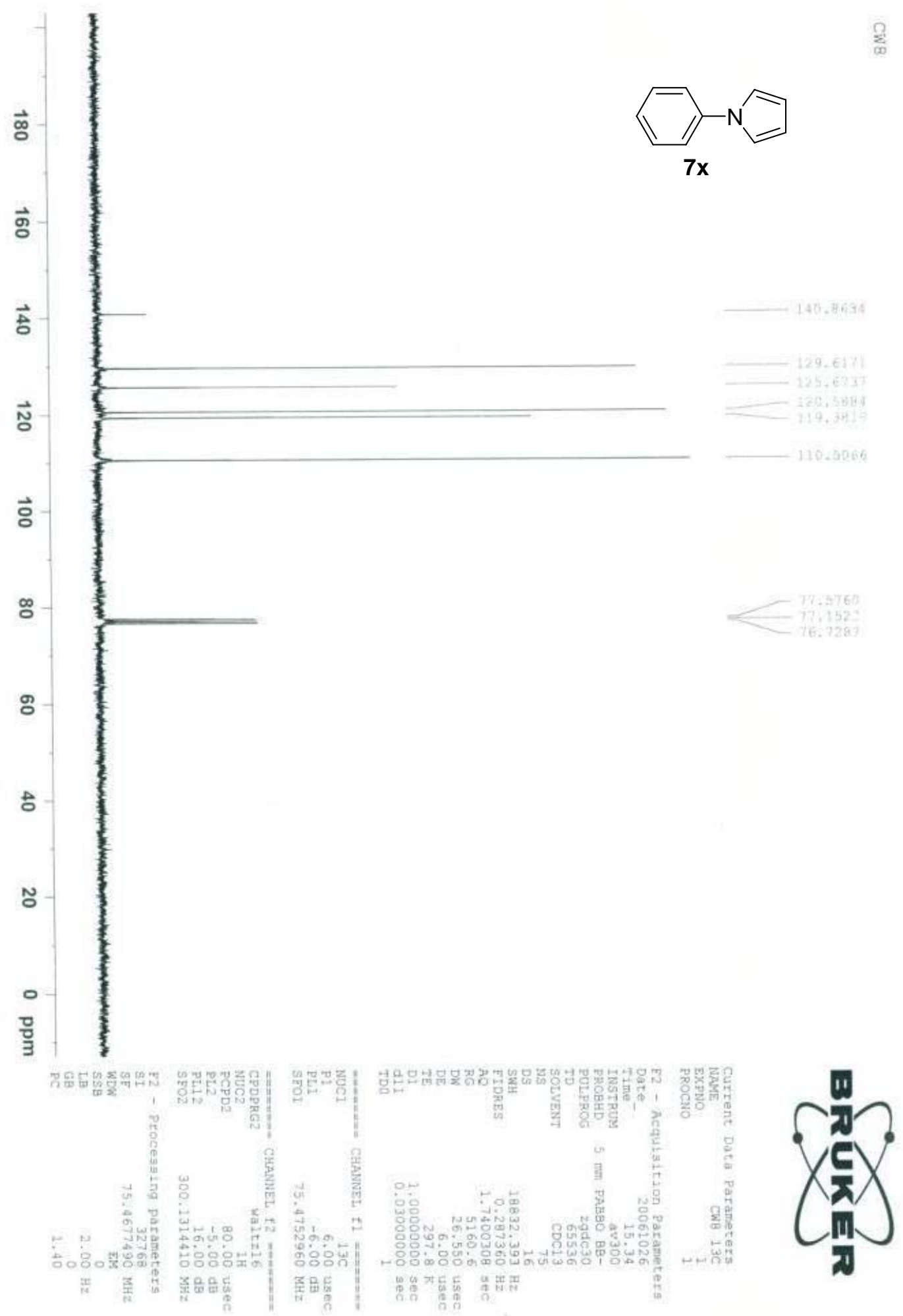




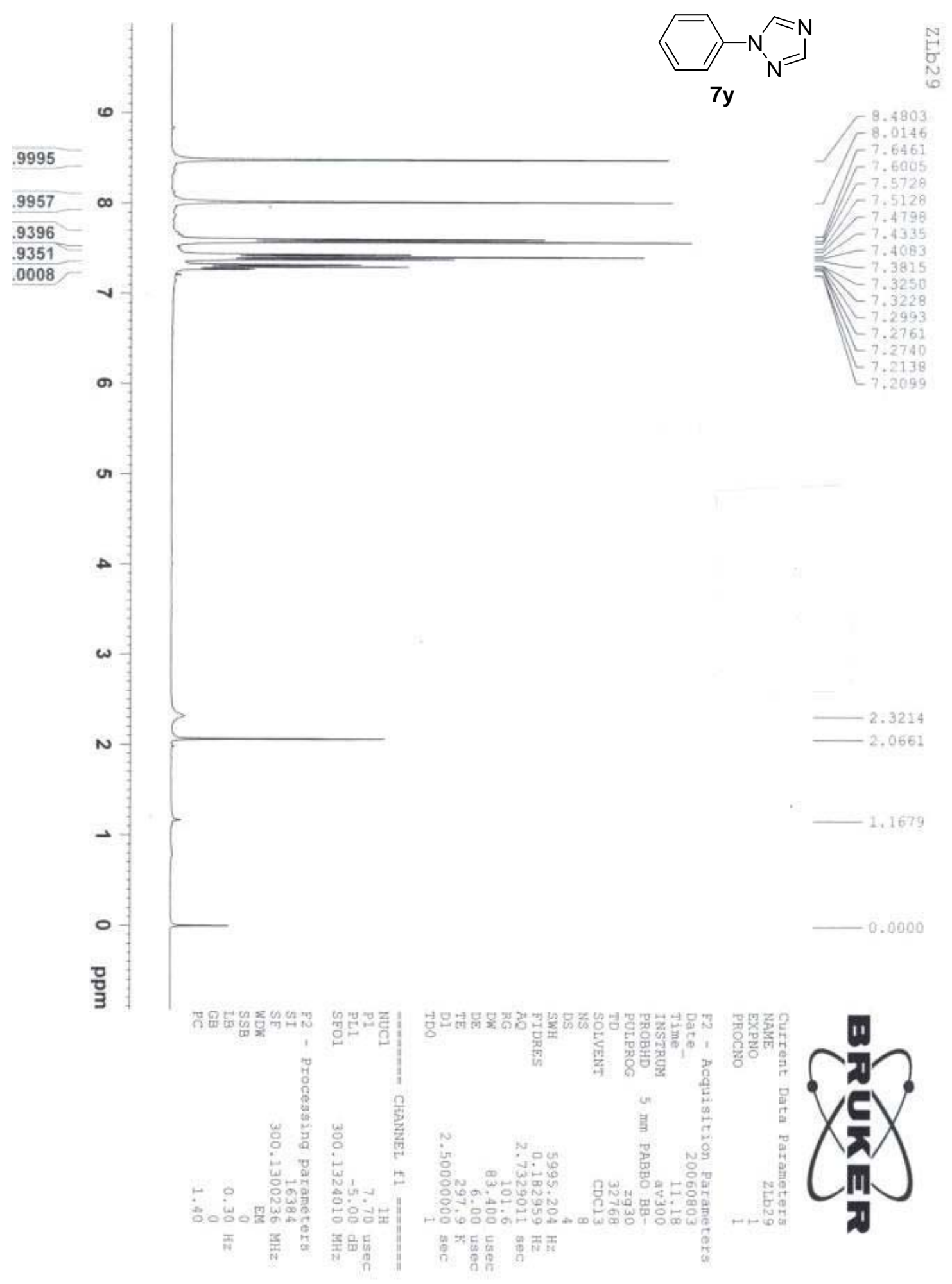




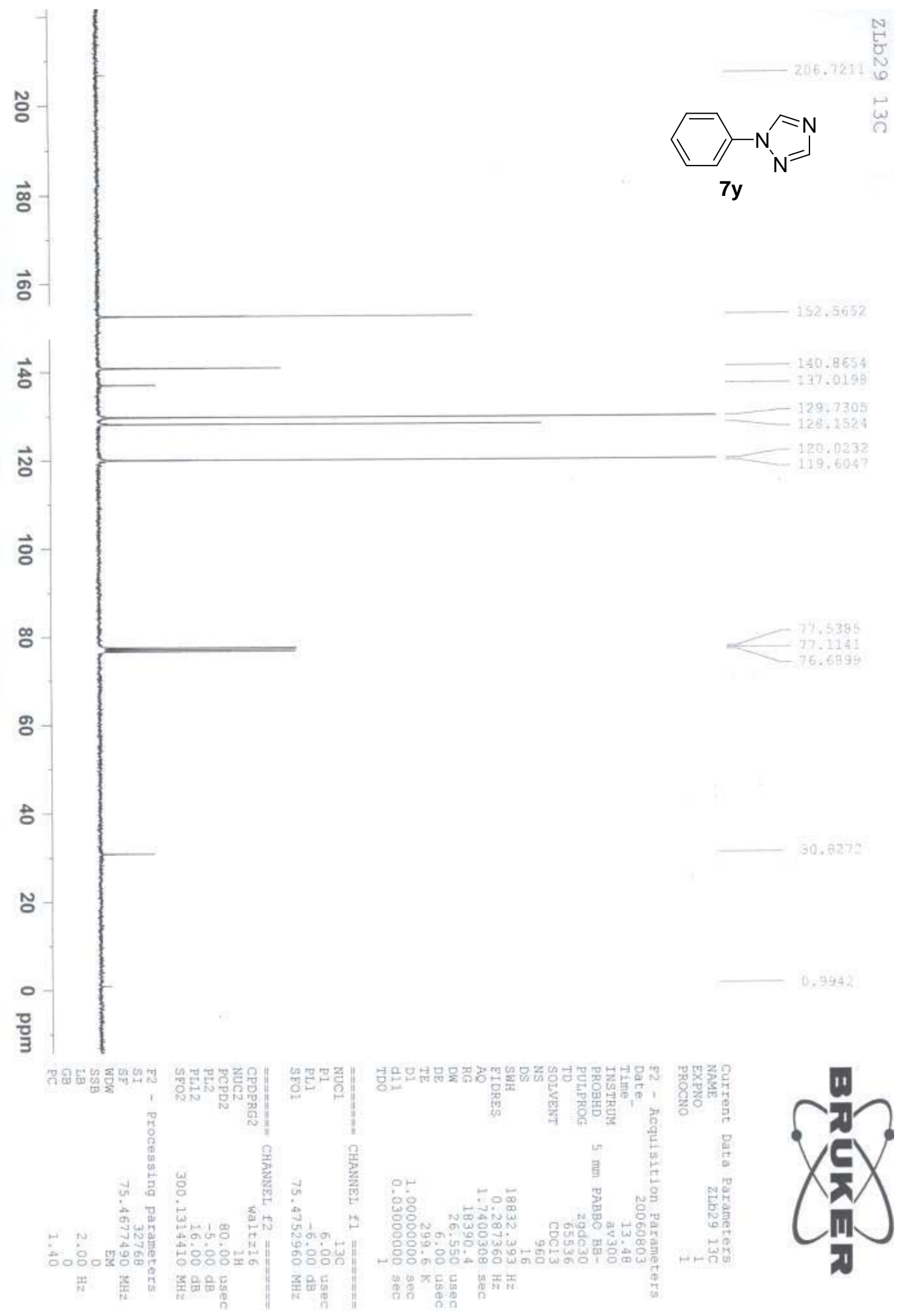




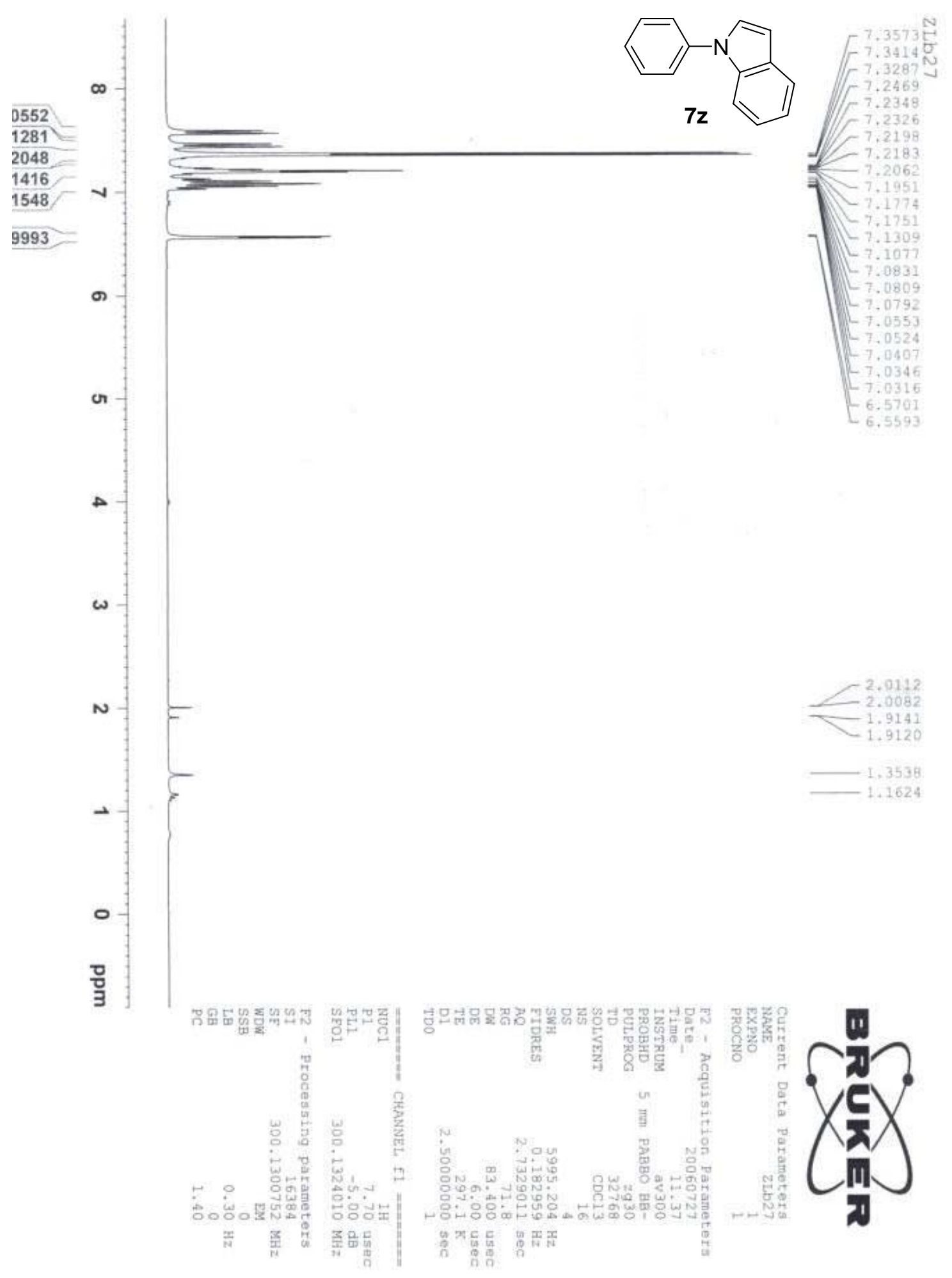




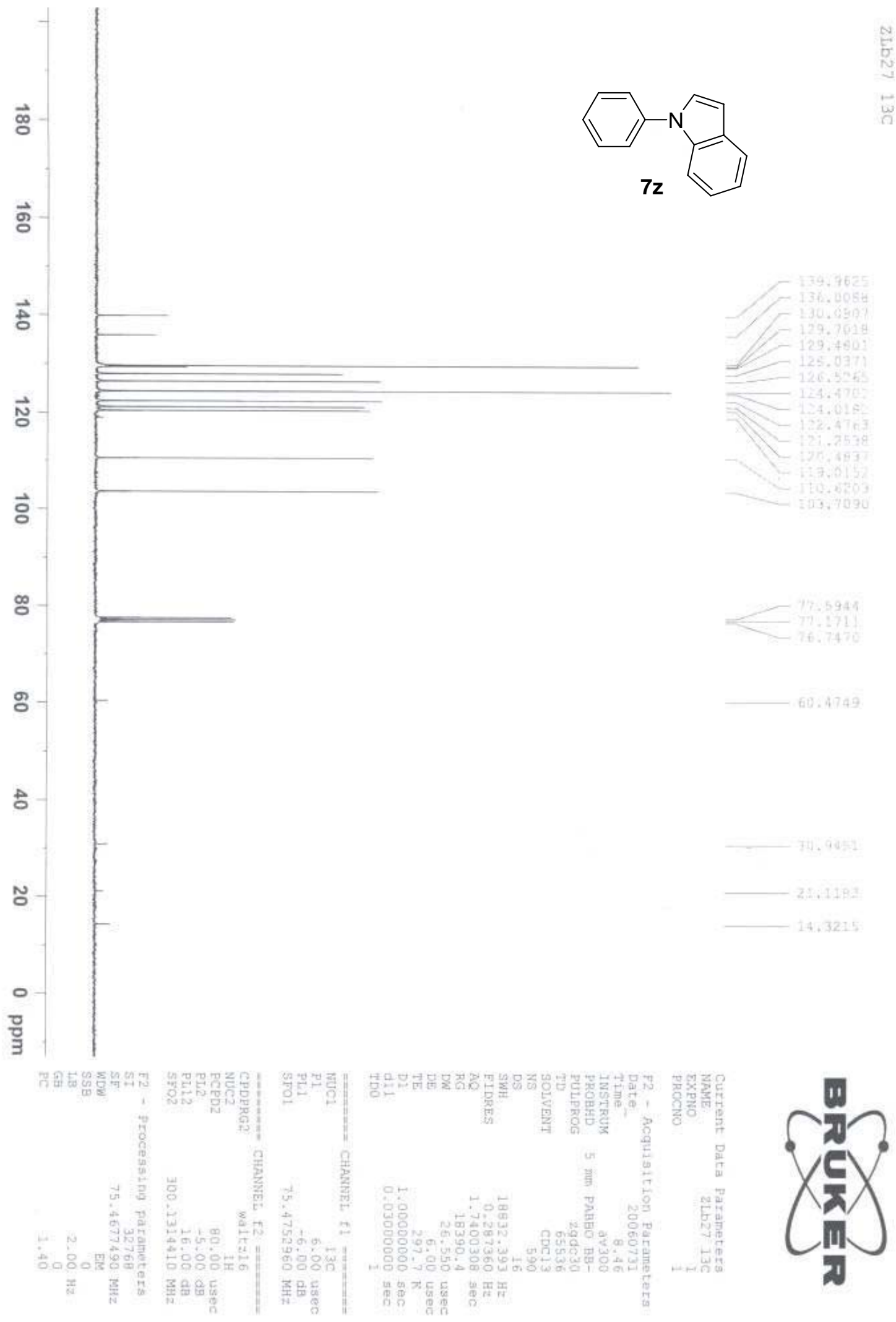

